Doctoral Dissertation

Academic Year 2009

\title{
Modeling Three-Dimensional Spatial Regulation of Bacterial Cell Division
}

\author{
Keio University

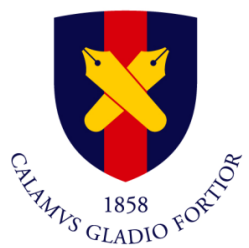 \\ Graduate School of Media and Governance \\ Keio University \\ SATYA NANDA VEL ARJUNAN
}




\title{
Modeling Three-Dimensional Spatial Regulation of Bacterial Cell Division
}

\author{
by \\ Satya Nanda Vel Arjunan \\ Bachelor of Engineering in Electronics Engineering \\ Universiti Teknologi Malaysia, 2000 \\ Master of Science in Computer Science \\ Universiti Teknologi Malaysia, 2003
}

\author{
Submitted to the \\ Graduate School of Media and Governance \\ in partial fulfillment of the requirements for the degree of \\ Doctor of Philosophy \\ at \\ KEIO UNIVERSITY
}

December 2009 
To my wife 


\section{ABSTRACT}

Many important cellular processes are regulated by reaction-diffusion (RD) of molecules that takes place both in the cytoplasm and on the membrane. To model and analyze such multicompartmental processes, we developed a lattice-based Monte Carlo method, Spatiocyte that supports RD in volume and surface compartments at single molecule resolution. Stochasticity in $\mathrm{RD}$ and the excluded volume effect brought by intracellular molecular crowding, both of which can significantly affect RD and thus, cellular processes, are also supported. We verified the method by comparing simulation results of diffusion, irreversible and reversible reactions with the predicted analytical and best available numerical solutions. Moreover, to directly compare the localization patterns of molecules in fluorescence microscopy images with simulation, we devised a visualization method that mimics the microphotography process by showing the trajectory of simulated molecules averaged according to the camera exposure time. In the rod-shaped bacterium Escherichia coli, the division site is suppressed at the cell poles by periodic pole-to-pole oscillations of the Min proteins (MinC, MinD and MinE) arising from carefully orchestrated RD in both cytoplasm and membrane compartments. Using Spatiocyte we could model and reproduce the in vivo MinDE localization dynamics by accounting for the previously reported properties of MinE. Our results suggest that the MinE ring, which is essential in preventing polar septation, is largely composed of MinE that is transiently attached to the membrane independently after recruited by MinD. Overall, Spatiocyte allows simulation and visualization of complex spatial and reaction-diffusion mediated cellular processes in volumes and surfaces. As we showed, it can potentially provide mechanistic insights otherwise difficult to obtain experimentally. Keywords: MinDE, diffusion, stochasticity, crowding, multicompartment 


\section{ACKNOWLEDGMENTS}

I thank Professor Martin Robert for guiding me in research and being a positive role model since the beginning of my stay in Japan. I hope in the future if I have my own students, I will be as genuinely concerned as he is of their research and welfare. I appreciate Professor Masaru Tomita for providing me the crucial support for my studies at Keio University and creating a very conducive environment for research in a small town such as Tsuruoka. His approach in encouraging graduate students to be independent and responsible of their own research has greatly inspired and motivated me. I thank Dr Koichi Takahashi for introducing me to the E-Cell System, his advice on research and for always looking out for my best interests.

I appreciate the views from my thesis committee members, Professor Mitsuhiro Itaya and Professor Yasuhiro Naito. I am also grateful to all the Keio University professors and staff - Ayumi Mikami and Akiko Shiozawa have gone beyond their call of duty to help me get settled to the life in Japan and fulfill my obligations as a Keio graduate student. I appreciate the kind help and the company of my friends at the Tsuruoka Town Campus of Keio University, especially Likai Mao, Dr Hu Bin, Lucy Zhang, Dr Qiu Dongru, Dr Aminul Hoque, Bereczki Gabor, Dr Richard Baran, Dr Masahiro Sugimoto, Takeshi Sakurada, Kenjiro Kami, Dr Azusa Kuroki, Dr Yuri Matsuzaki, Dr Nozomu Yachie, Anton Kratz, Mohammed Helmy, Kalesh Sasidharan, Cornelia Amariei, Vincent Piras and Kentaro Hayashi. I appreciate the generous support from the Japanese Government through the Monbukagakusho Scholarship Program and the Tsuruoka City for funding my research assistantship.

I am deeply grateful to my family for their continuous care and support. I do not think there ever was a time when my parents did not try to fulfill my requests. I greatly admire my father for being able to attend to any 
problem that he is faced with - he is truly an old school engineer. My mother is the mother every child hopes for - being born her son is my greatest fortune. When I was back in Malaysia, every morning without fail she would prepare the breakfast and bring it to my workstation. Her circadian clock is the most sensitive and reliable system that I have seen in a person, and I think I may have inherited it. My brothers, Siva and Nathan have been the voice of reason and have never failed to show me the big picture whenever I felt like I am lost. I am proud to have a caring and independent young sister like Vani.

My wife, Gracy has been the greatest source of strength, support and motivation although we were separated by distance throughout my time in Japan. My feeling of loneliness in Tsuruoka was only mitigated by her, even if it was only via Skype. I am truly thankful that I had met her on a corridor of my undergraduate alma mater over a decade ago and that she has stuck with me after all these years. 
TABLE OF CONTENTS

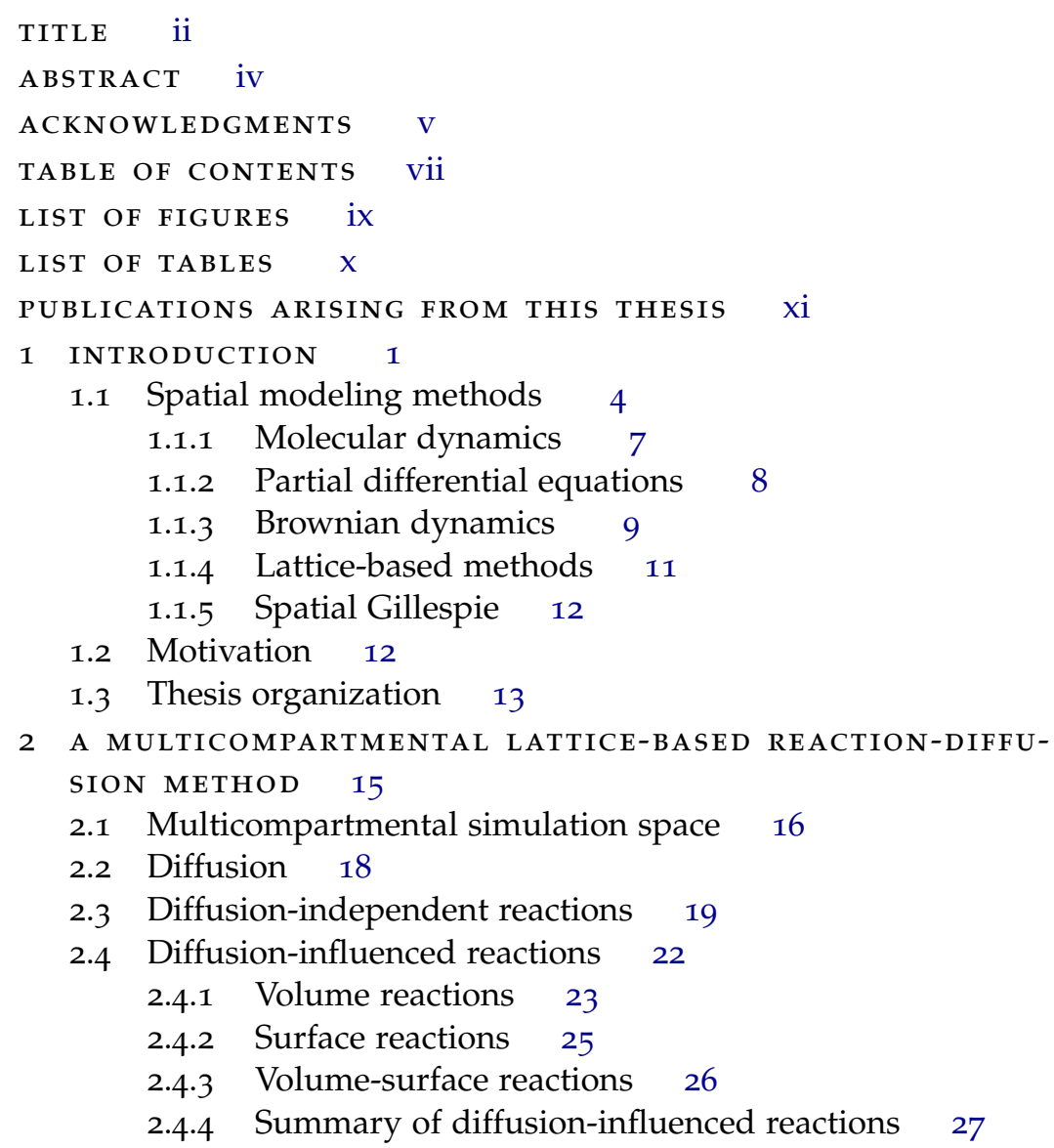

2.7 Simulating fluorescence microscopy images 32

2.8 Results 33

2.8.1 Diffusion in volume and surface compartments is verified 33

2.8.2 Reactions in volume and surface compartments are verified 33

2.8.3 Spatiocyte reproduces implications of molecular crowding $\quad 36$

2.8.4 Spatiocyte visualization method simulates microscopy images 40

2.9 Discussion 41

3 implementation of Spatiocyte With the E-CELl System 44

3.1 Methods 45

3.1.1 The reaction-diffusion scheme 
3.1.2 E-Cell System data structure and driver algorithm 47

3.1.3 Implementation of reaction-diffusion with E-Cell $\quad 48$

3.2 Application results 49

3.2.1 The MinDE Model 49

3.2.2 Simulation Results 50

3.3 Conclusions 56

4 MECHANISM OF E-RING FORMATION 58

4.1 Modeling the E-ring 60

4.2 Results 62

4.2.1 A wide range of model parameters reproduce MinDE

oscillation dynamics 62

4.2.2 Transient membrane attachment of MinE leads to the formation of E-ring $\quad 64$

4.2.3 Width of E-ring is proportional to MinD ATPase activation rate 68

4.3 Discussion 70

5 MODELING MEMBRANE-BOUND POLYMERIZATIONS 76

5.1 Methods 78

5.2 Results 80

5.2.1 Spatiocyte reproduces multistranded membrane-bound polymerizations 80

5.2.2 Spatiocyte reproduces various polymer curvatures on the membrane 84

5.3 Discussion 84

6 CONCLUSIONS AND FUTURE DiRECTIONS 86

BIBLIOGRAPHY 89

INDEX 103 
Figure 1.1 Computational modeling methods corresponding to system abstraction levels 2

Figure 1.2 Spatial representation schemes 5

Figure 2.1 Multicompartmental simulation space discretized into hexagonal close-packed (HCP) lattice 17

Figure 2.2 Methods for determining the average lateral displacement $r_{s}$ for a surface walk and the average number of voxels $n_{s}$ spread across an area $S \quad 20$

Figure 2.3 Verification of diffusion in volume and surface compartments 34

Figure 2.4 Verification of irreversible reactions in volume and surface compartments 35

Figure 2.5 Verification of reversible reactions in volume and surface compartments 37

Figure 2.6 Implications of molecular crowding in diffusion and reaction processes 38

Figure 2.7 Comparison of single molecule visualization to simulated microscopy image 41

Figure 3.1 The simplified oscillation model of the MinDE sytem in Escherichia coli 46

Figure 3.2 The data structure of the E-Cell Model. 48

Figure 3.3 Simulated cytosolic molecules of the MinDE system 53

Figure 3.4 MinDE oscillations on the membrane of Escherichia coli 54

Figure 3.5 Initial patches of MinD (cyan) and MinE.MinD $\mathrm{ATP}^{\mathrm{m}}$ (green) forming on the membrane (gray) 56

Figure 4.1 Comparison of MinDE polar zone and E-ring oscillations in wild type cells to simulation 66

Figure 4.2 Comparison of MinDE polar zone oscillation in mutant cells to simulation 68

Figure 4.3 The MinE ${ }^{\mathrm{D} 45 \mathrm{~A} / \mathrm{A}_{49} \mathrm{~A}}$ mutant dimer and molecular models of MinE interactions 72

Figure 5.1 Multistranded polymerization on a cylindrical membrane 81

Figure 5.2 Polymer curvatures on a spherical membrane 82

Figure 5.3 Polymer curvatures on a rod-shaped membrane 83 
LIST OF TABLES

Table 1.1 Features of spatial modeling methods 6

Table 3.1 Parameters of the simple MinDE model 52

Table 4.1 Parameters of the MinDE model to reproduce Ering 63 
Permission was obtained from the publishers, where necessary, to reproduce the published contents in this thesis.

- Takahashi, K., Arjunan, S. N. V. and Tomita, M. Space in systems biology of signaling pathways-towards intracellular molecular crowding in silico. FEBS Lett., 579(8):1783-1788, 2005. (Chapter 1)

- Arjunan, S. N. V. and Tomita, M. A new multicompartmental reactiondiffusion modeling method links transient membrane attachment of E. coli MinE to E-ring formation. Syst. Synth. Biol., (In press), 2009. (Chapters 2 and 4 )

- Arjunan, S. N. V. and Tomita, M. Modeling reaction-diffusion of molecules on surface and in volume spaces with the E-Cell System. Int. J. Comput. Sci. Inf. Secur., 3(1):211-216, 2009. (Chapter 3) 


\section{INTRODUCTION}

Molecular systems biology is a field that is concerned with understanding the processes in a cell as a unit rather than as the sum of its parts (Ideker et al., 2001; Kitano, 2002). Since the processes involve a network of biomolecular interactions, direct reasoning or intuition alone may not be adequate to understand them, and computational modeling and analysis, which are the integral parts of systems biology, become necessary. As shown in Figure 1.1, methods for computational modeling correspond to the level of abstraction or specificity of a system (Ideker and Lauffenburger, 2003). At the top level of abstraction, where qualitative features of a system are usually represented by the model, statistical mining techniques such as hierarchical clustering (Eisen et al., 1998; Brown et al., 2000) and artificial intelligence approaches such as support vector machines (Ramaswamy et al., 2001) help to reveal the key components of the model and potential interlinks by connecting dependent with independent variables. In the network, the conditional dependency of a child node on a parent can be determined using Bayesian networks (Sachs et al., 2002, 2005) while Boolean (Huang and Ingber, 2000) and fuzzy-logic models (Ressom et al., 2003) help to predict the logical rules regulating the dependencies. At a lower level of abstraction, where quantitative information of the system is more relevant, hidden Markov models (Riley et al., 2008) can probabilistically model the creation, removal and interconversion of molecular species and states, whereas when we are concerned with the rates of biochemical reactions, ordinary differential equations (ODE) (Bever, 2008; Boyce, 2008) can be used. Finally, at the bottom level abstraction, the structural information of the system and the dynamic localization of molecules, resulting from diffusion and intracellular transport, can be modeled using spatial modeling methods such as partial differential equations (PDEs), spatial 
Gillespie, lattice-based approaches, Brownian dynamics and molecular dynamics (reviewed in Takahashi et al., 2005; Ridgway et al., 2006).

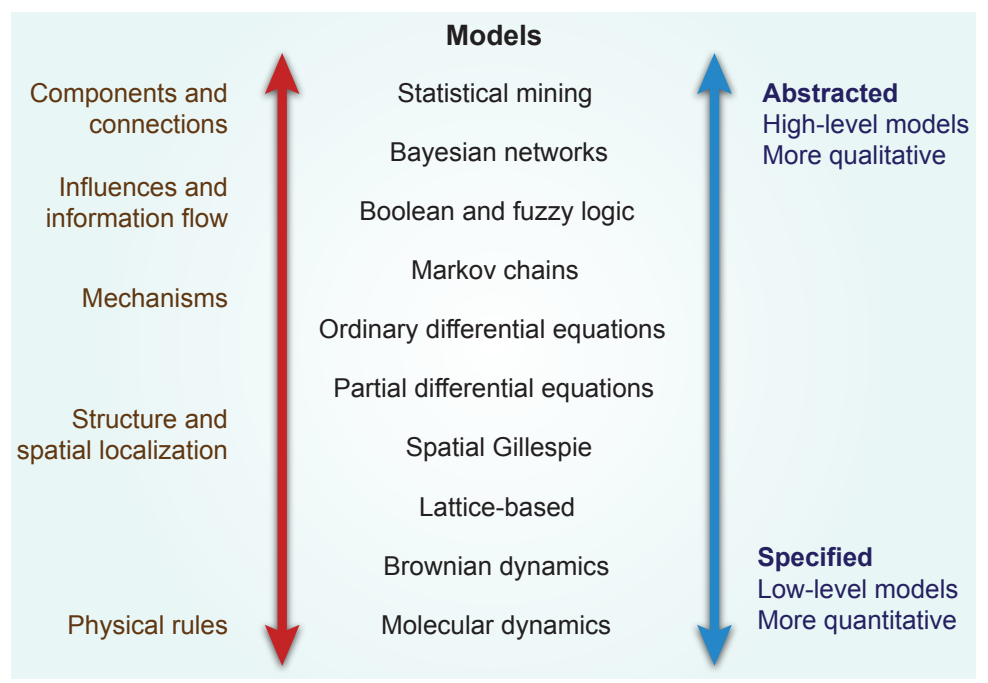

Figure 1.1: Computational modeling methods corresponding to system abstraction levels. The prominent computational methods to model a system are listed in the Models column. The methods are arranged according to the abstraction levels of the target system, as shown by the blue arrow. Highly abstract methods are listed at the top while increasingly specified models are arranged toward the bottom. The left column shows the type of information that can be gathered at various abstraction levels (indicated by the red arrow) using the corresponding methods. Highly specified models suffer from high computational cost although more detailed information can be gathered. Hence, as the methods become less abstract, the size of the target system needs to be reduced to cope with the increasing computational cost. Adapted from (Ideker and Lauffenburger, 2003) with permission from Elsevier, (c) 2003.

Some of the fundamental processes in the cell, such as cell division and signaling, require models with the lowest level of abstraction because they are strongly dependent on the structural information and the dynamic location of molecules. In the rod-shaped bacterium Escherichia coli for example, the Min system of proteins (Lutkenhaus, 2007), comprising MinC, MinD and MinE, oscillates from one pole to the other to direct the position of division to the middle of the cell long axis. By dividing in the middle, the cell ensures equal distribution of its contents to the two 
daughter cells. The proteins can oscillate in the presence of ATP because of the carefully orchestrated reaction and diffusion in the cytoplasm and on the inner membrane of the cell. As a result, without reaction, diffusion and the structural information of the cell, it is not possible to model the system accurately. Recently, computational models showed that although the proteins were initially uniformly distributed in the cell, the stochasticity in reaction-diffusion (RD) triggers the oscillation (Fange and Elf, 2006).

In signaling processes, extracellular cues captured by receptor proteins on the cell membrane are transduced inward to regulate target proteins or gene expression. Molecular diffusion and the signal transduction reactions are the two interconnected underpinnings of the cellular response to the cues. The biophysical properties of the cell structure and environment can influence diffusion, and by introducing non-linear delays, contribute to the overall signaling characteristics of the system. The significance of diffusion in $\mathrm{RD}$ systems is pronounced when reactions are comparatively faster than diffusion rates, i.e., the reactions are diffusion-limited. To this end, the Stokes-Einstein relation (Einstein, 1905) indicates that proteins, which are the key components in the signaling, have a slow diffusion rate because they are dense macromolecules. The phosphorylation state of target molecules with spatially separated membrane protein kinases and cytosolic phosphatases also depends heavily on diffusion (Kholodenko et al., 2000). In addition, subcompartments diffusively formed by localized proteins can significantly alter the effects of noise on signaling (Bhalla, 2004), implying the important coupling of noise and diffusion.

Very high protein density in the intracellular space, commonly called molecular crowding, can magnify the spatial effects. In a typical cell, the total macromolecular density is $50-400 \mathrm{mg} / \mathrm{ml}$ (Fulton, 1982), much higher than typical in vitro values of $1-10 \mathrm{mg} / \mathrm{ml}$. If identical globular proteins occupy $30 \%$ of the volume of a solution, less than $1 \%$ of the remaining space is available to an additional molecule of the same size because of steric repulsion (Zimmerman and Trach, 1991). The mutual 
impenetrability of macromolecular solutes is called the excluded volume effect. In such environments, the observed translational diffusion rate of proteins is about 5-20 times slower than in saline solutions (Elowitz et al., 1999). The anomalous diffusion, defined as sub-linear scaling of meansquared displacement of the molecule over time, is used as a measure for cytoplasmic crowding (Weiss et al., 2004). Molecular crowding can also alter protein activities and reaction kinetics (Ridgway et al., 2008). For reviews about the influence of molecular crowding on thermodynamics and volume exclusion, including experimental findings and biochemical reactions in physiological media, see (Minton, 2001) and (Hall and Minton, 2003).

Taken together, cellular processes involving molecular interactions that are tightly dependent on RD and spatial features of the cell require lowlevel and highly specific modeling methods. The methods should not only incorporate $\mathrm{RD}$ and structural information, but also the effects from molecular crowding and stochasticity in the simulations. In the next section, we review the features of existing spatial modeling methods.

\subsection{SPATIAL MODELING METHODS}

Spatial modeling methods can be broadly categorized into molecular dynamics, PDEs, Brownian dynamics, lattice-based and spatial Gillespie. Figure 1.2 illustrates the different kinds of approaches used by the methods to incorporate space when modeling a system, while Table 1.1 summarizes their features. We briefly discuss the methods in the following subsections. 

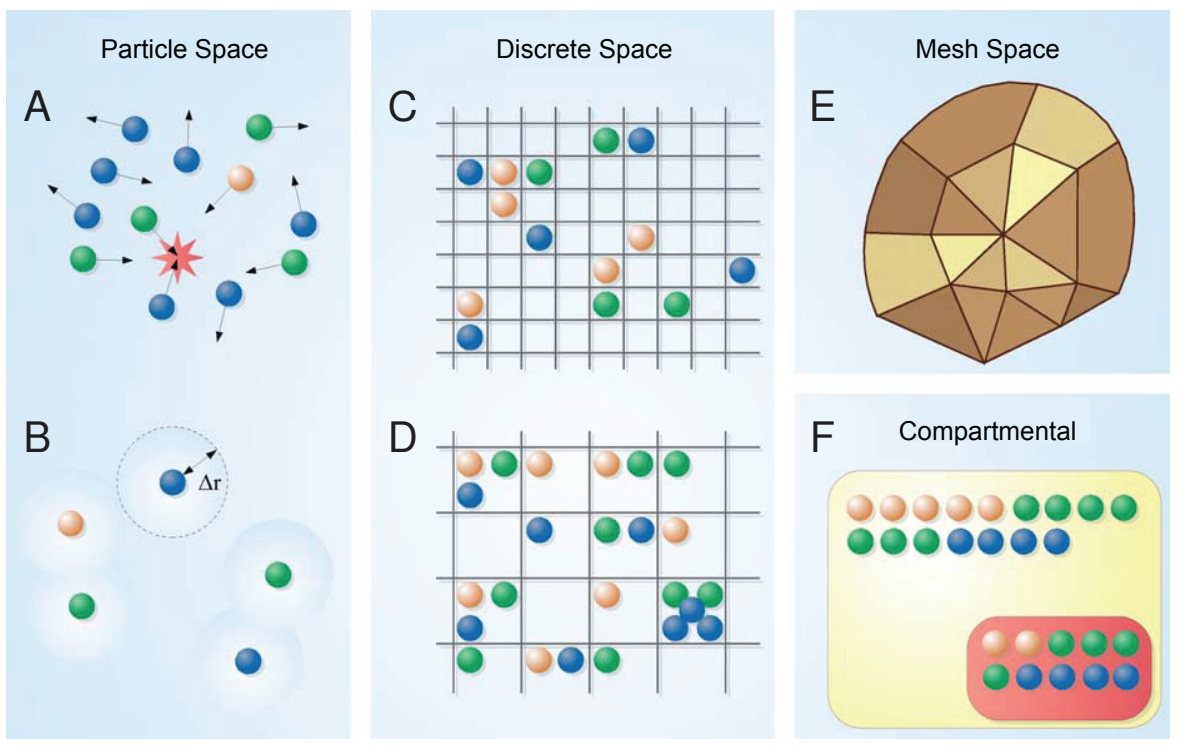

Figure 1.2: Spatial representation schemes. In particle space, molecules are represented as individual particles with positions in a continuum space. (A) Particles are usually given motions according to some kind of force equations that are numerically integrated to advance time. Reactions are represented as collisions between particles. (B) Some methods including GFRD employ an optimization technique that allows particles to jump in time and space by calculating the maximum distance $\Delta r$ that the particle can travel in the time slot. Discrete space representation discretizes the space either by subvolumes (voxels) of an identical shape (typically cubic) or a regular lattice. (C) In this microscopic lattice, at most one particle is allowed to occupy a lattice site. (D) Some methods allow multiple particles to reside in a single lattice site. This class of discrete space representation is called mesoscopic. (E) Mesh space here means conventional structured or unstructured meshing schemes of a concentration field. (F) Nonspatial biochemical simulators usually make use of compartmental space, which assumes a chemical equilibrium in each compartment, and molecular transfers between compartments are not modeled as implicit built-in rules in the simulation method (such as diffusion), but in an explicit way such as membrane transporters. Adapted from (Takahashi et al., 2005) with permission from the Federation of European Biochemical Societies, (C) 2005. 
Table 1.1: Features of spatial modeling methods.

\begin{tabular}{|c|c|c|c|c|c|c|}
\hline Method & $\begin{array}{c}\text { Spatial } \\
\text { Representation }\end{array}$ & $\begin{array}{l}\text { Spatial } \\
\text { Scale }\end{array}$ & $\begin{array}{l}\text { Temporal } \\
\text { Evolution }\end{array}$ & Stochasticity & $\begin{array}{c}\text { Excluded } \\
\text { Volume }\end{array}$ & $\begin{array}{c}\text { Multi } \\
\text { RD }\end{array}$ \\
\hline Virtual Cell (Schaff et al., 2001) & Gradient & Macro & DES & - & - & + \\
\hline MCell (Stiles and Bartol, 2001) & Continuum + Mesh & Micro+Meso & DT & + & - & + \\
\hline MesoRD (Elf and Ehrenberg, 2004) & Lattice & Meso & DEV & + & - & + \\
\hline Smoldyn (Andrews and Bray, 2004) & Continuum & Micro & DT & + & - & + \\
\hline SmartCell (Ander et al., 2004) & Lattice & Meso & DEV & + & - & + \\
\hline GFRD (van Zon and ten Wolde, 2005a) & Continuum & Micro & DEV & + & + & - \\
\hline GMP (Rodríguez et al., 2006) & Lattice & Meso & DEV & + & - & + \\
\hline Cell++ (Sanford et al., 2006) & Continuum+Gradient & Micro+Macro & DT & + & - & + \\
\hline CyberCell (Ridgway et al., 2008) & Continuum & Micro & DT & + & + & - \\
\hline GridCell (Boulianne et al., 2008) & Lattice & Micro & DT & + & + & - \\
\hline STEPS (Wils and Schutter, 2009) & Mesh & Meso & DEV & + & - & + \\
\hline eGFRD (Takahashi et al., 2009) & Continuum & Micro & DEV & + & + & - \\
\hline Spatiocyte (this work) & Lattice & Micro+Meso & $\mathrm{DT}+\mathrm{DEV}$ & + & + & + \\
\hline
\end{tabular}


Table 1.1: Features of spatial modeling methods (cont.). This table, adapted and updated from our review in (Takahashi et al., 2005), summarizes the features of current computational methods employing spatial representations to model biochemical pathways. Spatial Representation: in continuum space, molecular species are represented by individually treated discrete molecules that can move freely in a compartment, whereas in lattice space, discrete molecules occupy and move between regular subvolumes or voxels within a lattice. Mesh space represents discrete molecules in irregular triangulated (MCell) or tetrahedral (STEPS) surfaces, or tetrahedral volumes (STEPS). Gradient space denotes lattice (Cell++) or mesh (Virtual Cell) discretization scheme of the species concentration field. MCell uses continuum and mesh spaces for volume and surface molecules respectively, whereas Cell++ employs continuum and gradient spaces for large and small molecules respectively. Spatial Scale: in microscopic methods, each instance of molecule is distinguished from others and modeled as an object with a position either in continuum or lattice space. Macroscopic schemes represent the species state as a concentration gradient. There are many possible mesoscopic schemes between macro and micro realms. Mesoscopic methods in this table treat molecules discretely, but do not track positions in a compartment or within a subvolume. Temporal Evolution: simulation time-stepping scheme. $D E V, D T$, and DES mean event-driven, time-driven, and numeric solution of a continuous differential equation system, respectively. Stochasticity: if the method is stochastic. Excluded Volume: if the method can reproduce the excluded volume effect brought by molecules with physical dimensions in a crowded compartment (Zimmerman and Trach, 1991; Minton, 2001; Hall and Minton, 2003). Multicompartmental RD: if the method can perform RD in volumes and on surfaces, and between volume and surface compartments.

\subsubsection{Molecular dynamics}

Motions and interactions regulating all molecules constituting the cell arise from fundamental physical rules. By computing the forces affecting every molecule from many-body potentials in a particle space (Figure 1.2A) and numerically integrating Newton's laws over a small discrete time-step, the molecular dynamics (MD) approach can potentially be used to compute the macroscopic behavior of molecules in a system (Allen and Tildesley, 1989). However, the computational cost of MD simulation increases linearly 
with the number of interacting atoms (Rottler and Maggs, 2004). Despite being the most accurate and fundamental approach (Koplik and Banavar, 1995), MD cannot be used to simulate whole cell systems, which consist of very large number of atoms arising from macromolecules. It has only been used in problems involving time-scales of nanoseconds and space-scales of tens of nanometers. For example, it was employed to illustrate the effects of crowding in a cell on a small number of molecules (Baynes and Trout, 2004; Friedel and Shea, 2004).

\subsubsection{Partial differential equations}

Whereas the MD simulation approach deals with reaction and diffusion at the molecular level (i.e. microscale), the spatial partial differential equations (spatial PDEs) approach computes the intracellular kinetics at the macroscopic level. The Virtual Cell (Schaff et al., 1997, 2001; Slepchenko et al., 2003) employs PDEs with the finite volume method (Smith, 1986) to perform reaction and diffusion of mobile molecules in its spatial simulation framework. Compartments in the framework can depict the spatial structures of the system. These compartments are further divided into finite subvolumes through a mesh-generator (Figure 1.2E), and numerical methods are used to solve the differential equations. Finer time-step and subvolume sizes produce more accurate solutions but with higher computational overhead. Despite being one of the most computationally scalable spatial simulation algorithms, PDEs cannot accurately represent intracellular noise because it is a deterministic approach. Noise has profound implications especially when the number of molecules is small, as in the case of transcription factors (Rao et al., 2002). Moreover, noise is further amplified in finite subvolumes such as the one used by the Virtual Cell because molecule numbers in each subvolume will be smaller than when they are taken as a whole (Bhalla, 2004). Therefore, stochastic based simulation approaches should be considered in such conditions. Next, we 
look at other methods which are more sophisticated than spatial PDEs, but unlike MD, are still computationally tractable.

\subsubsection{Brownian dynamics}

Brownian dynamics (BD) is a stochastic modeling approach employing continuum space and time. In this particle based approach (Figure 1.2A), the molecules exhibit noise as they are propagated according to the Langevin equation (Coffey et al., 2004). The equation contains random forces that are intended to represent the interaction between the diffusing and the implicitly represented solvent molecules. BD has been applied successfully to investigate electrostatic competition effects between substrates binding to an enzyme (Elcock, 2002) and to observe how crowder molecules influence the GroEL-GroES chaperonin machinery at the atomic scale (Elcock, 2003). Hence, this approach can effectively simulate crowded environment, given that the crowder molecules are explicitly represented in the simulation space. Such representation, however, will incur very high computational costs, owing to the increased frequency of collision events and the smaller time-steps required to resolve them.

$\mathrm{BD}$ can be viewed as a numerical procedure to solve the Smoluchowski equation (von Smoluchowski, 1917), which describes the diffusive encounter of molecules in solution. On the other hand, for two-body problems, it is possible to analytically solve the equation by using the Green's function (Economou, 2006). This approach was adopted by van Zon and ten Wolde $(2005 \mathrm{a}, \mathrm{b})$ when they developed an event-driven simulation algorithm called Green's function reaction dynamics (GFRD) (Figure 1.2B). The basic idea is to reduce the many-body problem that constitutes the biochemical system into a set of two-body problem by determining the length of the time step to be sufficiently small. Although GFRD permits larger time-steps when the particles are too far apart to react, this advantage is lost when simulating crowded environments. This is because 
GFRD retains the drawbacks of BD, which is the dependency of step sizing scheme to the frequency of collision events. Nonetheless, this method can represent the excluded volume effect and represent different sizes and shapes of molecules. Smoldyn is another approach to numerically realize the Smoluchowski model of diffusion-limited reactions (Andrews and Bray, 2004). The molecules are represented as point particles (Figure 1.2A) with binding and unbinding radii, which are computed from the macroscopic reaction rate of each species. A disadvantage of discrete-time approaches in continuum space such as Smoldyn is that it is possible to miss collisions when the length of time-steps are not sufficiently small. Smoldyn can represent reduced diffusion speed in crowded environment by placing impenetrable blocks in space (Lipkow et al., 2005). One of the major consequences of the excluded volume effect is the dependency of the diffusive movements on physical sizes of the diffusing molecules. Unlike GFRD, dimensionless particles used in Smoldyn do not permit accurate representation of the effect. CyberCell shares the same properties of Smoldyn but the molecules can take different sizes (Ridgway et al., 2008). As a result, it can reproduce the excluded volume effect in crowded media. However, unlike Smoldyn, the present version of CyberCell does not support RD on surfaces. MCell is a another BD simulation method that can perform RD in both volume and surface compartments (Stiles and Bartol, 2001). For surface RD, the space is discretized into convex polygon meshes as illustrated in Figure 1.2E, whereas for reactions in the solution, it uses regular meshes to reduce computational cost when resolving molecular collisions. Similar to Smoldyn, MCell cannot model the effects of molecular crowding because the molecules are represented as point particles. 


\subsubsection{Lattice-based methods}

The cellular automata (CA) method employs a lattice of uniform voxels with a finite number of states that evolves in discrete-time (Weimar and Boon, 1994; Alber et al., 2003). The transition of each automaton (i.e., molecule) in the voxels is fully specified by its local interaction. The molecule can propagate either along its velocity vector or according to its diffusion rate to arrive at another voxel, and then collide or react with other molecules. CA can be used to simulate RD at both microscopic (Figure 1.2C) (Berry, 2002; Schnell and Turner, 2004; Wishart et al., 2005; Boulianne et al., 2008) and mesoscopic (Figure 1.2D) spatial scales, having single and multiple molecules in a voxel, respectively. Using lattice-based methods, the effects of molecular crowding can be simulated when the spatial scale is microscopic and the size of voxels follows the molecule size. Lattice size and geometry (e.g., square, hexagonal or trigonal) can also influence the outcome of simulation, as reported by Shimizu et al. (2003) when they analyzed the Escherichia coli chemotaxis signaling pathway using a CA-based Ising model. Large differences in molecule sizes and numbers in the cells motivated Weimar (2002) to use CA to simulate enzymatic reaction networks at both mesoscale (metabolites) and microscale (enzymes) simultaneously on a two-dimensional lattice. This approach considerably reduces the memory requirement, especially when considering large systems such as the cell. The size of the voxels can be larger to accommodate large molecules, and as a result, fewer sites will need to be created and stored in the memory. The reduced resolution of the lattice would, however, translate to lower precision of the molecular diffusion at each time-step. The local interaction nature of CA makes it suitable for implementation on parallel architectures and hence, supports reduction in the computational time required for $3 \mathrm{D}$ simulations. To our knowledge, all microscopic lattice-based methods have not successfully modeled volume-surface RD. 


\subsubsection{Spatial Gillespie}

Stundzia and Lumsden (1996) extended the Gillespie's stochastic approach (Gillespie, 1976, 1977) to be used in subvolumes for spatial modeling. Their method was employed to model the propagation of a calcium waves by RD across a cell. Elf and Ehrenberg (2004) on the other hand, extended the fast version of the Gillespie's algorithm, the Next Reaction (NR) method (Gibson and Bruck, 2000), to be used in subvolumes. The SmartCell (Ander et al., 2004) and GMP (Rodríguez et al., 2006) simulators also implemented a similar scheme. The subvolume sizes, as shown in (Figure 1.2D), are determined such that all reactive molecular species, represented as point particles, are almost uniformly distributed in each subvolume's space. This is done by ensuring that the diffusion of reactants in a subvolume takes place more frequently (e.g., more than 1oo times) than their respective reactions. At each time-step, each molecule can either react in its current subvolume or diffuse to an adjacent one. The diffusional probability at each time-step is obtained by mapping the bulk diffusion constant in Fick's law using the Green's function. Similar to the original NR method, the computation time increases only logarithmically with the number of subvolumes in the system. Nonetheless, it is not possible to reproduce crowded conditions because volume exclusion from both reactive and non-reactive crowder molecules cannot be represented explicitly when they are depicted as point particles.

\subsection{MOTIVATION}

Ideally, spatial modeling methods should be able to run simulations with minimal computational cost because the simulation time usually presents as a bottleneck when a system is modeled at such high specificity. To this end, discrete-event methods are promising because computation is only performed at time steps when it is really necessary and as a result, larger jumps in time is possible. In addition to stochasticity in simulation and 
the effects of volume exclusion, a spatial modeling method should also be able to perform RD on the surface, and between volume and surface compartments. This feature becomes essential when modeling cellular processes such as the bacterial cell division. However, as can be seen in Table 1.1, none of the present methods have all the expected features.

In this thesis, we aim to develop a new lattice-based modeling method, called Spatiocyte, which is sufficiently fast to run many simulation runs of actual cellular processes. The method should be stochastic and able to model the implications of molecular crowding. RD in the volume and surface compartments should also be supported so that fundamental cellular processes such as the division site control in bacteria can be modeled accurately. Results from spatial modeling efforts are usually verified by comparing with experimental data using images of in vivo localization of fluorescent labeled proteins. Since the images are highly dependent on the exposure time of camera, a visualization method needs to be developed to show the localization of simulated molecules that mimics the output of the camera to directly and effectively compare the result of simulations with that of experiments.

\subsection{THESIS ORGANIZATION}

In the Chapter 2 of this thesis, the detailed description of the proposed modeling method will be presented. To verify the correctness of the method, simulation results of basic RD in volume and surface compartments will be compared with analytical and numerical solutions. In addition, the new visualization method that mimics the fluorescent labeled protein images will be presented. In Chapter 3, the implementation details of the method using the E-Cell System will be described. The implemented method will be used to model the Escherichia coli MinDE dynamics and compared with the results from experiments and previous computational studies. In Chapter 4 , the method is used to elucidate the mechanism of 
E-ring formation in Escherichia coli. In Chapter 5, we describe the extension of the Spatiocyte method to model polymerization of molecules on surfaces such as cell and nuclear membrane. Finally, in Chapter 6, the overall conclusions and potential future directions of the research presented in this thesis will be provided. 
Computational models are valuable to both theoretical and experimental biologists to rapidly analyze and test their predictions about qualitative or quantitative behavior of complex cellular processes (Ideker and Lauffenburger, 2003). Moreover, the models can potentially provide mechanistic insights (Treanor and Batista, 2007; Clarke and Liu, 2008; Neves and Iyengar, 2009), even in the case of relatively simple processes (Stefan et al., 2008; van Albada and ten Wolde, 2009).

Processes that are strongly dependent on the cell morphology, multicompartmental interaction and dynamic localization of molecules such as cell signaling (Rangamani and Iyengar, 2008; Neves et al., 2008), bacterial cell division (Lutkenhaus, 2007; Adams and Errington, 2009) and chemotaxis (Greenfield et al., 2009; Rao and Ordal, 2009), require realistic models with spatial representation (Takahashi et al., 2005; Ridgway et al., 2006; Morris and Jensen, 2008). The processes rely on intricately controlled reaction and diffusion of molecules in and between cytoplasm (or nucleoplasm) and membrane compartments to perform their functions. Excluded volume effect brought by molecular crowding in each compartment can reduce the macroscopic diffusion coefficient and alter reaction kinetics (Ellis, 2001; Hall and Minton, 2003; Dix and Verkman, 2008; Klann et al., 2009). Furthermore, the significance of slower diffusion in such reaction-diffusion (RD) processes is pronounced when the reactions are diffusion-limited. Since some of the species involved in the processes comprise small number of molecules, stochasticity in the reactions can dominate over the law of mass action (Gillespie, 1976; Gómez-Uribe and Verghese, 2007), and thus affecting the macroscopic behavior of the processes (Fange and Elf, 2006; Rangamani and Iyengar, 2008; Hansen et al., 2008). 
In Chapter 1 we have looked at the prominent spatial modeling methods with a summary of their features listed in Table 1.1. The table shows that although a variety of methods have been developed, modeling cellular processes at the level where multicompartmental RD, molecular crowding and stochasticity all simultaneously come into play is still not possible with any single method. To address this issue, in this chapter we develop a general-purpose hybrid time- and event-driven, lattice-based Monte Carlo simulation method, called Spatiocyte, to model processes that are dependent on multicompartmental RD, stochasticity and the implications of molecular crowding.

\subsection{MULTICOMPARTMENTAL SIMULATION SPACE}

In our method, molecules can participate in first- or second-order reactions, each giving away one or two product molecules. Free molecules can diffuse within the simulation space either on a surface or in a volume compartment. In vivo, where the compartments are crowded, diffusing protein molecules collide even at very small time scales (Ellis, 2001). The advantage of fully event-driven reaction-diffusion methods to make large jumps in time when molecules are far apart (van Zon and ten Wolde, 2005b) is lost in such conditions (Ridgway et al., 2006; Takahashi et al., 2005). Instead, we use a time-driven, lattice-based method to skip two costly operations - molecule search and distance calculation - required by off-lattice methods (Andrews and Bray, 2004; van Zon and ten Wolde, 2005b; Ridgway et al., 2008) to resolve collisions. Diffusion-independent reactions, however, are event driven to further reduce the computational cost.

We discretized the space into a hexagonal close-packed (HCP) lattice where each sphere voxel has 12 adjoining but non-overlapping neighbors (Figure 2.1A). Each voxel is at most occupied by a single molecule to account for volume exclusion and molecular crowding. The voxel radius $r_{v}$ is uniform and set to the radius of individually simulated molecules. 

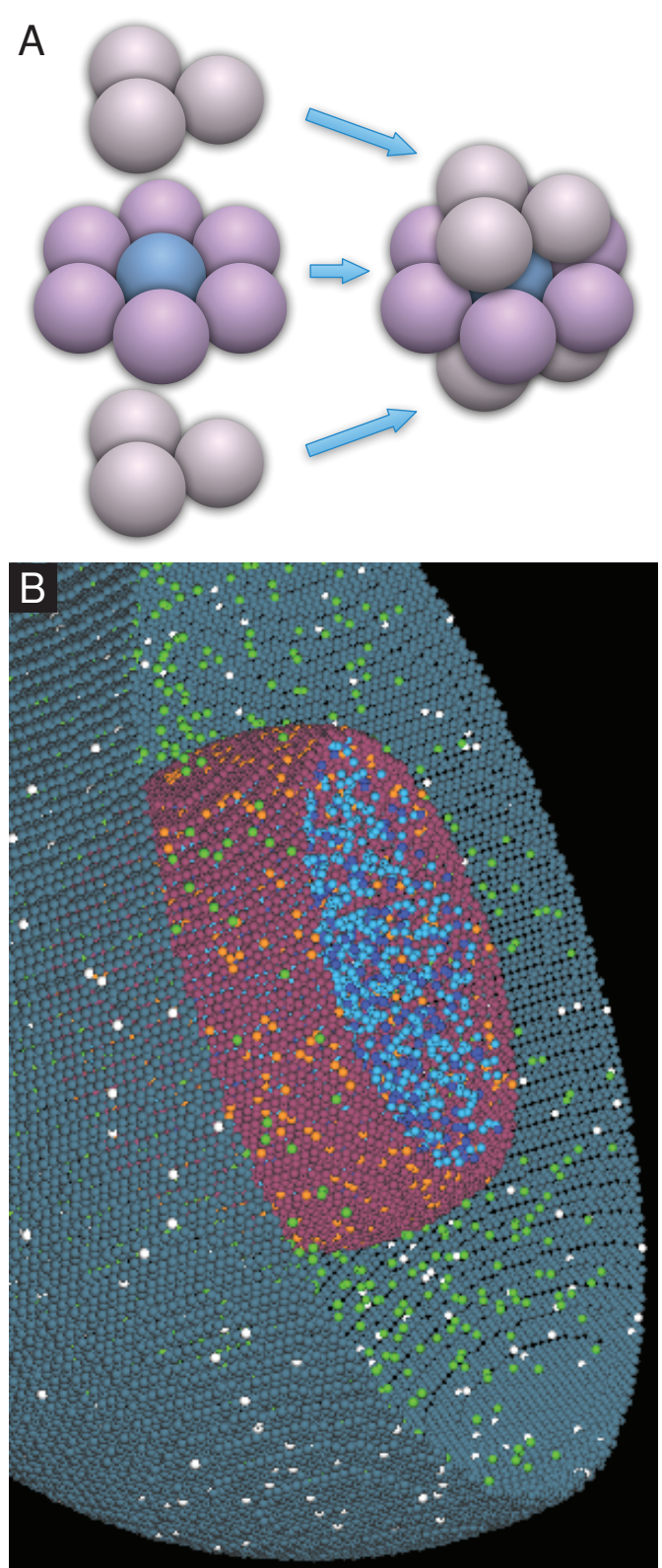

Figure 2.1: Multicompartmental simulation space discretized into hexagonal close-packed (HCP) lattice. (A) A voxel in the HCP lattice (blue) has 12 adjoining neighbor voxels. (B) Cross-section of cell (teal) and nuclear (maroon) membranes represented by immobile lipid molecules that constrain molecules to their respective compartment. Other colored spheres represent molecules in their respective voxel. Various geometric primitives such as boxes, spheres and cylinders can be used in combinations to define compartment structures, cell shapes and intracellular geometries, which may affect the model behavior. 
Compared to the lattice arrangements of other reaction-diffusion methods (Novère and Shimizu, 2001; Ander et al., 2004; Elf and Ehrenberg, 2004; Rodríguez et al., 2006; Sanford et al., 2006; Boulianne et al., 2008), the HCP lattice has the highest density of sphere voxels (Szpiro, 2003). To delineate a surface compartment such as a cell or a nuclear membrane, we occupy all empty voxels of the compartment with immobile lipid molecules (Figure 2.1B). Analogous to in vivo, these molecules aid lateral diffusion of non-lipid surface species, while together as an immobile structure, sequester volume species.

In addition to the diffusing and immobile species that are treated individually at the molecular scale, there are also homogeneously distributed (HD) species, such as ATP, that are simulated at the compartmental scale. We consider a species HD only if it is mobile and evenly distributed within the compartment before its reaction events. The species can satisfy these conditions if its reaction events are not limited by its diffusion rate. Alternatively, it can have a sufficiently high concentration that, after each reaction event, even if it has a slower diffusion rate it can rapidly attain a homogeneous concentration before the next event. Each molecule of the HD species is not treated individually since the compartmental concentration information is sufficient. For a biochemical species $A$, we denote $A^{\#}$ as the total molecule number in a compartment. All random numbers are drawn independently from the uniform distribution.

\subsection{DIFFUSION}

Applying the Einstein-Smoluchowski expression for diffusion $\left\langle\mathrm{r}^{2}\right\rangle=$ $2 l D_{i} \tau_{i}$ (Einstein, 1905; von Smoluchowski, 1906), with $l=3$ for volume diffusion, a molecule $i$ with diffusion coefficient $D_{i}$ can walk to a randomly selected neighbor voxel in a time step $\tau_{i}$ when the mean squared displacement $\left\langle\mathrm{r}^{2}\right\rangle=\left(2 \mathrm{r}_{v}\right)^{2}$ (Rodríguez et al., 2006). For a surface molecule, the walk is constrained to adjoining surface voxels, $l=2$ and the average 
voxel-to-voxel lateral displacement is $\frac{2(2 \sqrt{2}+4 \sqrt{3}+3 \sqrt{6}+\sqrt{22}) \mathrm{r}_{v}}{6 \sqrt{2}+4 \sqrt{3}+3 \sqrt{6}} \approx 1.915 \mathrm{r}_{v}$ (calculated in Figure 2.2). Thus, if $t_{s}$ is the current simulation time, the next time a diffusing molecule walks is given by

$$
t_{d}^{i}=t_{s}+\frac{\alpha_{i} r_{v}{ }^{2}}{D_{i}}
$$

where $\alpha_{i}=\frac{2}{3}$ or $\alpha_{i}=\left(\frac{2 \sqrt{2}+4 \sqrt{3}+3 \sqrt{6}+\sqrt{22}}{6 \sqrt{2}+4 \sqrt{3}+3 \sqrt{6}}\right)^{2}$ for volume or surface species, respectively. However, a volume molecule can only occupy the target voxel if it is vacant, otherwise a collision occurs and the walk fails. A surface walk is only successful if the target voxel is occupied by a lipid molecule, in which case the lipid and the walking molecule exchange voxels; a collision occurs if the target voxel contains a non-lipid surface species. The above scheme accurately constrains molecules to their own compartment without geometry calculations.

Molecules sharing a diffusion coefficient $D_{i}$ are grouped in a diffusion process $d_{i}$ which executes their random walk together at $t_{d}^{i}$. In long timescales, diffusion becomes a costly operation because of the very short time steps required to make voxel-length random walks. To alleviate this cost, every voxel has an address pointer to each of its neighbors as a rapid means of access when checking for vacancy and molecule species. In addition, only mobile molecules undergoing diffusion-influenced reactions, usually found in small numbers, are individually diffused, while others are simulated at the compartmental scale as HD species.

\subsection{DIFFUSION-INDEPENDENT REACTIONS}

$A \stackrel{k_{A}}{\longrightarrow}$ product(s) is a first-order reaction with the product(s) comprising diffusing, immobile or HD species. It can represent a unimolecular reaction, which can be decoupled from the diffusion process since $k_{A}$ is only dependent on time. It can also denote a bimolecular reaction (i.e., pseudo first-order), involving $A$ and an unrepresented HD species with a fixed 


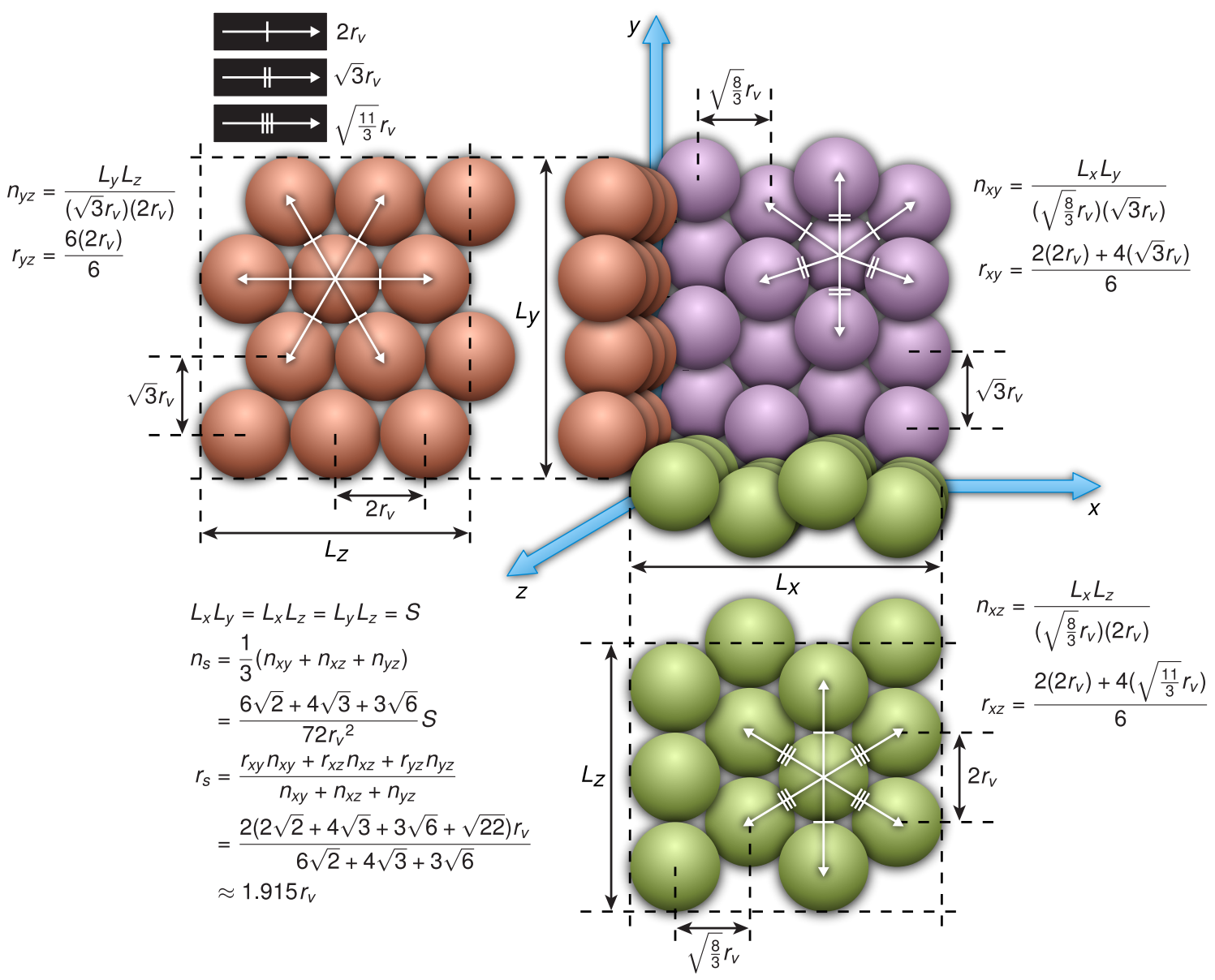

Figure 2.2: Methods for determining the average lateral displacement $r_{s}$ for a surface walk and the average number of voxels $n_{s}$ spread across an area $S$. The spheres in purple, green and red show the voxel arrangement of the HCP lattice that are delineated as surface voxels to form the planes $x y, x z$ and $y z$ respectively. Some of the voxels are flatly spread (yz-plane), while others are jagged and overlap when they represent the flat surfaces ( $x y$ - and $x z$-planes). The lateral displacement of a molecule walking voxelto-voxel on the planes, shown as white arrows in six directions, can be less than $2 r_{v}$ because of the overlaps. As a result, to calculate the surface diffusion step interval $\tau_{d}^{i}$ (see text) we use the average lateral displacement $r_{s}$ that is computed from the average displacement on the three planes, $r_{x y}, r_{x z}$ and $r_{y z}$. Similarly, $n_{s}$ is calculated from the average number of voxels spread across the three planes, $n_{x y}, n_{x z}$ and $n_{y z}$.

concentration. The reaction is decoupled from diffusion by setting $k_{A}$ as the asymptotic reaction rate given by $k_{a} \pi R D /\left(k_{a}+4 \pi R D\right)$, where $k_{a}$ is 
the intrinsic reaction rate, while $R$ and $D$ are the contact distance and combined diffusion coefficient, respectively.

Applying the asymptotic reaction rate, a second-order reaction involving a HD reactant can also be decoupled from the diffusion or immobility of the second reactant because before the reaction event, the concentration of the HD species is the same at any location of the compartment. Likewise, if both reactants are immobile, as long as they are in contact (via adjoining voxels in the simulation), the rate of reaction is also unaffected. Taken together, we can decouple diffusion from all first-order reactions, and all second-order reactions that involve two adjoining immobile reactants or at least one HD reactant.

We perform event-driven diffusion-independent reactions by adapting the NR method (Gibson and Bruck, 200o), a computer-optimized variation of the Gillespie algorithm (Gillespie, 1976, 1977) that stores reaction dependencies in a directed graph and the time of next reactions in an indexed priority queue. The steps in an iteration of the NR method are as follows: (i) select the reaction $\mathrm{m}$ with the earliest next reaction time $t_{r}^{m}$ from the priority queue; (ii) execute reaction $m$ and update species molecule numbers; (iii) using the dependency graph, get every reaction $\mu$ whose propensity $a_{\mu}$ is affected by the updated species and recompute it:

$$
a_{\mu}= \begin{cases}k_{A} A^{\#,} & A \stackrel{k_{A}}{\longrightarrow} \operatorname{product}(s), \\ k_{S} A^{\#} S, & A \stackrel{k_{S}}{\longrightarrow} \operatorname{product}(s), \\ \frac{k_{A B}}{V} A^{\#} B^{\#}, & A+B \stackrel{k_{A B}}{\longrightarrow} \operatorname{product}(s), \\ \frac{k_{A A}}{V} A^{\#}\left(A^{\#}-1\right), & A+A \stackrel{k_{A A}}{\longrightarrow} \operatorname{product}(s),\end{cases}
$$

with $S$ the surface compartment area, $k_{S}$ the rate of surface associations per surface area, and $V$ the compartment volume, which is replaced with $S$ if both reactants are surface species; and (iv) for each affected reaction, 
using a random number $u_{r}$ in the range $(0,1)$ update the priority queue with the new next reaction time

$$
\mathrm{t}_{r}^{\mu}=\mathrm{t}_{\mathrm{s}}-\frac{\ln \mathrm{u}_{\mathrm{r}}}{\mathrm{a}_{\mu}}
$$

The original method assumes all molecules to be HD species, which is not the case in our approach. As a result, in step (ii), if $m$ has a non-HD reactant, we perform the reaction with a molecule selected randomly from the reactant molecule list. A non-HD product of $m$ replaces a non-HD reactant at the same voxel. A second non-HD product will occupy a vacant neighbor voxel of the first product. A non-HD product of $m$ involving only HD reactants is placed in a random vacant voxel of the reaction compartment. If it is an intercompartmental reaction, the product will take up a vacant voxel adjoining both compartments. Because reactions are diffusion-independent, we can avoid partitioning reaction volumes like in other Gillespie-based spatial simulation methods (Elf and Ehrenberg, 2004; Ander et al., 2004; Rodríguez et al., 2006; Bernstein, 2005).

\subsection{DIFFUSION-INFLUENCED REACTIONS}

Only two remaining second-order reactions, involving a diffusing and an immobile reactant, and two diffusing reactants, that are diffusion influenced. They are modeled using a spatially discretized version of the Collins and Kimball approach (Collins and Kimball, 1949) that assigns a finite probability $p$ for a reactive collision between reactants. Next, we derive the relationship between the reactive collision probability $p$ and the macroscopic rate constant of various diffusion-influenced second-order reactions. 


\subsubsection{Volume reactions}

Consider an elementary reaction $A+B \stackrel{k_{A B}}{\longrightarrow} C$ that is diffusion-influenced and takes place in the HCP lattice. We first derive the connection between $p$ and $k_{A B}$ when both $A$ and $B$ are volume species. Since the density of HCP voxels is $\frac{\pi}{3 \sqrt{2}}$ (Conway and Sloane, 1998), the total number of voxels in a compartment volume $\mathrm{V}$ is

$$
\mathrm{n}_{v}=\frac{\frac{\pi}{3 \sqrt{2}} \mathrm{~V}}{\frac{4}{3} \pi \mathrm{r}_{v}^{3}}=\frac{\mathrm{V}}{4 \sqrt{2} \mathrm{r}_{v}{ }^{3}}
$$

At the beginning of simulation, the molecules are placed randomly in the voxels to represent a uniformly-distributed reaction volume. Molecule collisions for $A$ are evaluated by a diffusion process at the interval

$$
\tau_{\mathrm{A}}=\frac{\alpha_{\mathrm{A}} r_{v}^{2}}{\mathrm{D}_{\mathrm{A}}}
$$

where $D_{A}$ is the diffusion coefficient and $\alpha_{A}=\frac{2}{3}$ because $A$ is a volume species. The probability for an A molecule to collide with a B molecule at the target voxel in a small interval $\Delta t$ is

$$
\begin{aligned}
p_{A} & =\frac{B^{\#}}{n_{v}} \times \frac{\Delta t}{\tau_{A}}, \\
& =\frac{B^{\#}}{n_{v} \tau_{A}} \Delta t,
\end{aligned}
$$

if $n_{v} \gg 1$. Analogously, for each B

$$
\begin{aligned}
& \tau_{\mathrm{B}}=\frac{\alpha_{\mathrm{B}} \mathrm{r}_{v}^{2}}{\mathrm{D}_{\mathrm{B}}}, \\
& \mathrm{p}_{\mathrm{B}}=\frac{\mathrm{A}^{\#}}{\mathrm{n}_{v} \tau_{\mathrm{B}}} \Delta \mathrm{t},
\end{aligned}
$$


with $\alpha_{B}=\frac{2}{3}$. Since the collisions are evaluated for all $A$ and B molecules, the average number of collisions in $\Delta t$ is

$$
\begin{aligned}
Z & =A^{\#}\left(p_{A}\right)+B^{\#}\left(p_{B}\right), \\
& =\frac{A^{\#} B^{\#}\left(\tau_{A}+\tau_{B}\right)}{n_{v} \tau_{A} \tau_{B}} \Delta t .
\end{aligned}
$$

Of these collisions, if some are reactive and $\Delta \mathrm{C}$ is the change in the number of product molecules, then

$$
\begin{aligned}
p & =\frac{\Delta C}{Z}, \\
& =\frac{n_{v} \tau_{A} \tau_{B} \Delta C}{A^{\#} B^{\#}\left(\tau_{A}+\tau_{B}\right) \Delta t} .
\end{aligned}
$$

At the macroscopic level,

$$
\frac{d[C]}{d t}=k_{A B}[A][B]
$$

and in a very small $\Delta \mathrm{t}$ within the volume $\mathrm{V}$,

$$
\Delta \mathrm{C}=\frac{\mathrm{k}_{\mathrm{AB}} \mathrm{A}^{\#} \mathrm{~B}^{\#}}{\mathrm{~V}} \Delta \mathrm{t}
$$

Substitutions of (2.4), (2.5), (2.7) and (2.13) into (2.24) give

$$
p=\frac{k_{A B}}{6 \sqrt{2}\left(D_{A}+D_{B}\right) r_{v}} .
$$

Similarly, for the homodimerization reaction $A+A \stackrel{k_{A A}}{\longrightarrow} C$ we obtain 


$$
\begin{gathered}
Z=\frac{A^{\#}\left(A^{\#}-1\right)}{n_{v} \tau_{A}} \Delta t, \\
\Delta C=\frac{k_{A A} A^{\#}\left(A^{\#}-1\right)}{V} \Delta t,
\end{gathered}
$$

and substituting (2.4), (2.5), (2.15) and (2.16) into (2.10) yields

$$
p=\frac{k_{A A}}{6 \sqrt{2} D_{A} r_{v}}
$$

\subsubsection{Surface reactions}

We next consider reactions that take place on the surface compartment. As shown in Figure 2.2, the average number of voxels spread across the compartment with an area $S$ is

$$
\mathrm{n}_{\mathrm{s}}=\frac{6 \sqrt{2}+4 \sqrt{3}+3 \sqrt{6}}{72 \mathrm{r}_{v}^{2}} \mathrm{~S}
$$

For the surface reaction $A+B \stackrel{k_{A B}}{\longrightarrow} C$, we derive $p$ by applying the same strategy that we used for the volume reaction but because the reactants are surface species, $\alpha_{A}=\alpha_{B}=\left(\frac{2 \sqrt{2}+4 \sqrt{3}+3 \sqrt{6}+\sqrt{22}}{6 \sqrt{2}+4 \sqrt{3}+3 \sqrt{6}}\right)^{2}, n_{s}$ now substitutes $n_{v}$ in (2.6), (2.8), (2.9) and (2.24) while $S$ replaces $V$ in (2.13) to get

$$
p=\frac{(2 \sqrt{2}+4 \sqrt{3}+3 \sqrt{6}+\sqrt{22})^{2} k_{A B}}{72(6 \sqrt{2}+4 \sqrt{3}+3 \sqrt{6})\left(D_{A}+D_{B}\right)}
$$

Likewise, for the surface homodimerization reaction $A+A \stackrel{k_{A A}}{\longrightarrow} C$, we substitute $n_{v}$ with $n_{s}$ in (2.15) and $V$ with $S$ in (2.16) to obtain 


$$
p=\frac{(2 \sqrt{2}+4 \sqrt{3}+3 \sqrt{6}+\sqrt{22})^{2} k_{A A}}{72(6 \sqrt{2}+4 \sqrt{3}+3 \sqrt{6}) D_{A}}
$$

\subsubsection{Volume-surface reactions}

A volume molecule $A$ can also collide with a surface molecule $B$ and react with probability $p$. Since each B only diffuses laterally on the surface, it does not strike $A$ in the volume compartment.

The number of volume voxels adjoining the surface compartment on either side is also $n_{s}$. Each of these volume voxels has three adjoining surface voxels. The probability for an $A$ to collide with $B$ at the target voxel is $\operatorname{Pr}($ the molecule $A$ is occupying a voxel adjoining the surface compartment $) \times \operatorname{Pr}($ a surface voxel is selected as the target voxel $) \times \operatorname{Pr}($ target surface voxel contains $B) \times($ number of collision evaluations in the interval $\Delta \mathrm{t}$ ):

$$
\begin{aligned}
p_{A} & =\frac{n_{s}}{n_{v}} \times \frac{3}{12} \times \frac{B^{\#}}{n_{s}} \times \frac{\Delta t}{\tau_{A}}, \\
& =\frac{B^{\#}}{4 n_{v} \tau_{A}} \Delta t .
\end{aligned}
$$

To get $p$, we substitute $Z=A^{\#}\left(p_{A}\right)$ in (2.9) and apply the remaining derivation steps as in the volume reaction $A+B \stackrel{k_{A B}}{\longrightarrow} C$ :

$$
p=\frac{\sqrt{2} k_{A B}}{3 D_{A} r_{v}}
$$

Finally, a volume species $A$ can also attach itself to the surface and become a surface species $C$, as in $A \stackrel{k_{A}}{\longrightarrow} C$, where $k_{A}$ is the surfaceaverage rate coefficient with dimensions of velocity (Balgi et al., 1995). In the lattice, this is actually a second-order reaction $A+B \stackrel{k_{A B}}{\longrightarrow} C$, where $B$ 
is the lipid species of the surface compartment. Since each surface voxel by default is occupied by a lipid molecule, $B^{\#}=n_{s}$. To derive $p$ for the reaction between $A$ and $B$ in terms of $k_{A}$, we apply the same procedure as in the previous volume-surface reaction but we now replace $B^{\#}$ with $n_{s}$ in (2.21) and substitute $\Delta C=k_{A} A^{\#} S \Delta t / V$ in (2.13):

$$
p=\frac{24 k_{A} r_{v}}{(6+3 \sqrt{3}+2 \sqrt{6}) D_{A}}
$$

In contrast to the MCell (Stiles and Bartol, 2001) and GridCell (Boulianne et al., 2008) methods, in a time step, we only evaluate a single target voxel for collision, reducing the computational cost by several folds.

\subsubsection{Summary of diffusion-influenced reactions}

In summary, for a diffusion-influenced reaction $j$, the relationship between $p_{j}$ and the macroscopic rate constant of various diffusion-influenced second-order reactions is given as

$$
p_{j}= \begin{cases}\frac{k_{A B}}{6 \sqrt{2}\left(D_{A}+D_{B}\right) r_{v}}, & A_{v}+B_{v} \stackrel{k_{A B}}{\longrightarrow} \operatorname{product}(s), \\ \frac{k_{A A}}{6 \sqrt{2} D_{A} r_{v}}, & A_{v}+A_{v} \stackrel{k_{A A}}{\longrightarrow} \operatorname{product}(s), \\ \frac{\gamma k_{A B}}{D_{A}+D_{B}}, & A_{s}+B_{s} \stackrel{k_{A B}}{\longrightarrow} \operatorname{product}(s), \\ \frac{\gamma k_{A A}}{D_{A}}, & A_{s}+A_{s} \stackrel{k_{A A}}{\longrightarrow} \operatorname{product}(s), \\ \frac{\sqrt{2} k_{A B}}{3 D_{A} r_{v},}, & A_{v}+B_{s} \stackrel{k_{A B}}{\longrightarrow} \operatorname{product}(s), \\ \frac{24 k_{S} r_{v}}{(6+3 \sqrt{3}+2 \sqrt{6}) D_{A}}, & A_{v}\left(+L_{s}\right) \stackrel{k_{S}}{\longrightarrow} \operatorname{product}(s),\end{cases}
$$

where $\gamma=\frac{(2 \sqrt{2}+4 \sqrt{3}+3 \sqrt{6}+\sqrt{22})^{2}}{72(6 \sqrt{2}+4 \sqrt{3}+3 \sqrt{6})}$. The species subscripts $v$ and $s$ denote volume and surface species respectively, while $\mathrm{L}$ is the lipid species. 
If $j$ is diffusion-limited, a reactive collision may occur at time slices smaller than $\tau_{A}$, causing $p_{j}>1$ and yielding inaccurate results. Here, for a species $i$ that participates in J number of diffusion-influenced reactions, we limit its reaction probabilities to a maximum value $P_{i}, 0 \leqslant P_{i} \leqslant 1$, by reducing its diffusion process interval to $\tau_{i}^{\prime}=\tau_{i} P_{i} / \rho_{i}$, with $\rho_{i}=$ $\max \left\{p_{1}, \ldots, p_{J}\right\}$. Consequently, (2.1) becomes

$$
t_{d}^{i}= \begin{cases}t_{s}+\frac{\alpha_{i} r_{v}^{2}}{D_{i}}, & \rho_{i} \leqslant P_{i}, \\ t_{s}+\frac{\alpha_{i} r_{v}^{2} P_{i}}{D_{i} \rho_{i}}, & \rho_{i}>P_{i} .\end{cases}
$$

Note that if $\rho_{i}>P_{i}$, the walk probability is no longer unity because of the reduced interval and is given as $P_{i} / \rho_{i}$. However, at each interval the molecule can collide as usual with a neighbor reactant pair and react with probability $\mathrm{p}_{j} \mathrm{P}_{\mathrm{i}} / \rho_{i}$.

When the collision is reactive, the two reactants are removed and new non-HD product(s) occupy the reactant or neighbor voxels, whereas for HD product(s), only the species molecule number is updated. Since the reactant and product molecule numbers have changed, we also perform steps (iii) and (iv) of the adapted NR method for the diffusion-independent reactions.

\subsection{HYBRID TIME- AND EVENT-DRIVEN SIMULATION}

We adopt the multi-timescale algorithm of the E-Cell System (Takahashi et al., 2004) to concurrently run time- and event-driven processes (described in Chapter 3$)$. All $n$ number of $t_{d}^{i}$ and $t_{r}^{\mu}$ are stored in an indexed priority queue $Q$, which provides $t_{q}=\min \left\{t_{d}^{i}, t_{r}^{\mu}\right\}$ and can be updated in $\mathrm{O}(\log n)$ (Takahashi et al., 2004). If $t_{q}=t_{d}^{i}, d_{i}$ is executed and $Q$ is updated with a new next walk time from (2.25). If $t_{q}=t_{r}^{\mu}$, the steps (ii) to (iv) of the adapted NR method are executed using $\mathrm{Q}$ and with 
$m=\mu$. By incrementing $t_{s}=t_{s}+t_{q}$, we can run diffusion processes at predefined time steps while diffusion-independent reactions are executed in an event-driven manner.

2.6 VERIFICATION OF DIFFUSION-INFLUENCED REACTIONS

To verify the diffusion-influenced reactions of our method we employ a similar approach as in (Ridgway et al., 2008) by comparing the simulation results with the predicted survival probability of reacting species in irreversible $\left(S_{\text {irr }}\right)$ and reversible $\left(S_{\text {rev }}\right)$ reactions. Here, we describe the analytical and numerical methods to obtain $S_{\text {irr }}$ and $S_{\text {rev }}$ since they have not been previously described for surface reactions. Where possible we adopt the notations in (Popov and Agmon, 2001).

2.6.1 Irreversible reactions

According to the Smoluchowski theory (von Smoluchowski, 1917; Szabo, 1989; Popov and Agmon, 2001), in the irreversible reaction $A+B \stackrel{k_{A B}}{\longrightarrow} C$ with dilute $A$ and $B$, the fraction of $A$ still surviving at time $t$ is

$$
S_{\text {irr }}\left(t ; c_{0}\right)=\exp \left[-c_{0} \int_{0}^{t} k_{\text {irr }}\left(t^{\prime}\right) d t^{\prime}\right]
$$

where $c_{0}$ is the initial concentration of $B$ and $k_{\text {irr }}(t)$ is the time-dependent rate coefficient. For volume reactions,

$$
k_{\text {irr }}(t)=k_{D}^{\prime}\left[1+\frac{k_{A B}}{k_{D}} \Phi\left(\frac{k_{A B}}{k_{D}} \sqrt{\frac{t}{\tau_{D}^{\prime}}}\right)\right] .
$$


Here the function $\Phi(x)=\exp \left(x^{2}\right) \operatorname{erfc}(x), k_{D}=4 \pi\left(D_{A}+D_{B}\right) R$ is the diffusion-limited rate constant, $k_{D}^{\prime}=k_{A B} k_{D} /\left(k_{A B}+k_{D}\right)$ is the asymptotic reaction rate and

$$
\tau_{D}^{\prime}=\frac{1}{D_{A}+D_{B}}\left(\frac{k_{A B} R}{k_{A B}+k_{D}}\right)^{2}
$$

For reactions taking place in the HCP lattice, the contact distance $\mathrm{R}=2 \mathrm{r}_{v}$. The time-dependent rate coefficient is only known in the Laplace space for surface reactions,

$$
\tilde{k}_{\text {irr }}(s)=\frac{k_{A B}}{s\left[1+k_{A B} \tilde{G}(s)\right]},
$$

where

$$
\tilde{G}(s)=\frac{K_{0}\left(\sqrt{s \tau_{D}}\right)}{2 \pi\left(D_{A}+D_{B}\right) \sqrt{s \tau_{D}} K_{1}\left(\sqrt{s \tau_{D}}\right)}
$$

is the Green's function for free 2D diffusion (Popov and Agmon, 2002), obeying a reflective boundary condition at $R ; K_{n}(z)$ is the modified Bessel function of the second kind for the integer order $n$; and $\tau_{D}=$ $R^{2} /\left(D_{A}+D_{B}\right)$ is the diffusion-limited time constant. The transform (2.29) is numerically inverted to obtain $k_{\text {irr }}(t)$ and subsequently, the survival probability $S_{\text {irr }}\left(t ; c_{0}\right)$.

\subsubsection{Reversible reactions}

For the reversible reaction $A+B \underset{k_{C}}{\stackrel{k_{A B}}{\rightleftharpoons}} C$ with a single target $A$ and many $B$ molecules, the best known method to estimate the survival probability $S_{\text {rev }}(t)$ of $A$ is the first variant of the multiparticle kernel theories 
(MPK1) (Sung and Lee, 1999). The normalized deviation of $S_{\text {rev }}(t)$ from the predicted equilibrium value $S_{\text {rev }}(\infty)=k_{D} / \lambda$, with $\lambda=k_{C}+c_{0} k_{A B}$, is obtained from the inverse Laplace transform

$$
\frac{S_{\text {rev }}(t)-S_{\text {rev }}(\infty)}{S_{\text {rev }}(0)-S_{\text {rev }}(\infty)}=\mathcal{L}^{-1}\left\{\frac{\tilde{F}_{\mathrm{MPK}_{1}}(s)}{s \tilde{F}_{\mathrm{MPK}_{1}}(s)+\lambda}\right\}
$$

which is calculated numerically. $\tilde{\mathrm{F}}_{\mathrm{MPK}_{1}}(\mathrm{~s})$ is the diffusion factor function of the reversible reaction according to MPK1,

$$
\tilde{\mathrm{F}}_{\mathrm{MPK} 1}(\mathrm{~s})=\frac{k_{\mathrm{AB}}}{\lambda} \tilde{\mathrm{F}}_{\mathrm{gem}}(\mathrm{s})+\frac{\mathrm{c}_{0} k_{\mathrm{AB}}}{\lambda} \tilde{\mathrm{F}}_{\text {irr }}\left(s ; \mathrm{c}_{0}^{\prime}\right)
$$

where the modified concentration $c_{0}^{\prime}=\lambda / k_{A B}, \tilde{F}_{\text {gem }}(s)=1+k_{A B} \tilde{G}(s)$ is the diffusion factor function for a geminate pair, while in the irreversible case, the function is given as

$$
\tilde{\mathrm{F}}_{\mathrm{irr}}\left(s ; c_{0}\right)=\frac{c_{0} k_{\mathrm{AB}} \tilde{S}_{i r r}\left(s ; c_{0}\right)}{1-s \tilde{S}_{i r r}\left(s ; c_{0}\right)}
$$

The reflective Green's function in $2 \mathrm{D}$ is given in (2.30) whereas in $3 \mathrm{D}$ it is

$$
\tilde{\mathrm{G}}(\mathrm{s})=\frac{1}{\mathrm{k}_{\mathrm{D}}\left(1+\sqrt{\mathrm{s} \tau_{\mathrm{D}}}\right)}
$$

$\tilde{S}_{\text {irr }}\left(s ; c_{0}\right)$ in $(2.33)$ is obtained by numerically computing the Laplace transform

$$
\tilde{S}_{\text {irr }}\left(s ; c_{0}\right)=\int_{0}^{\infty} S_{\text {irr }}\left(t ; c_{0}\right) \exp (-s t) d t
$$


Spatial localization patterns of molecules both in vitro and in vivo are widely determined using fluorescence labeling experiments (Resch-Genger et al., 2008; Fernández-Suárez and Ting, 2008; Ji et al., 2008; Huang et al., 2009). Co-localization patterns of multiple species of molecules can also be determined using multiple fluorescent labels. The merged localization images show the blending of colors when the positions of the separately labeled molecules overlap. However, the optical microscopy images suffer from low resolution because of the diffracted light and the long exposure time required by the camera in microphotography processes. The exposure time of several hundred milliseconds to several seconds makes it impossible to capture the position of diffusing molecules at single molecule resolution. As a result, it is difficult to directly compare the position of molecules obtained from spatial simulations with the microscopy images.

Here, we develop a visualization method that simulates microscopy images by displaying the trajectory of simulated molecules averaged according to the exposure time. We use the OpenGL programming interface for $3 \mathrm{D}$ computer graphics (Shreiner, 2009) to develop the method. We adopt the RGBA mode of OpenGL, where the colors are represented by red, green, blue and alpha color components. The alpha color component allows a color to be blended with the existing color at the intended position. An alpha value of o implies full transparency, while an alpha value of 1 implies full opacity. The background color of the simulation space is set to black to represent the unlit space of the microscopy image. We record the voxel position of each molecule, regardless of its diffusion coefficient, at each minimum diffusion step interval $\tau_{m}=\min \left\{\tau_{i}\right\}$. For each voxel, the number of times a molecule of a fluorescent-labeled species $f$ has occupied it in the exposure time $\tau_{e}$ can then be counted, and is given as $N_{f}$. To display the trajectory of the labeled species $f$ within the exposure time, the alpha value of each voxel is calculated as 


$$
a=\frac{2 N_{f} \tau_{m}}{\tau_{e}},
$$

while its RGB values are set to the species fluorescent color. The above step is repeated for the remaining fluorescent labeled species. When molecules of separately labeled species occupy the same voxel within the exposure time, or overlap during visualization, their colors are blended according to their opacity levels. In addition, the intensity of voxel colors is directly proportional to the density of molecules at the position during the exposure time, as it is the case in microscopy images.

\subsection{RESULtS}

\subsubsection{Diffusion in volume and surface compartments is verified}

Figure 2.3 shows that the mean squared displacements of freely diffusing molecules in volume and surface compartments using Spatiocyte are indistinguishable from expected analytic values. The diffusion of the molecules is also correct when the walk probability $P_{i} / \rho_{i}$ is less than unity in a diffusion process interval $\tau_{i}^{\prime}$. Based on the results in Figure 2.3, the multi-timescale algorithm is also verified because the diffusion of all four species, in different compartment types and with unique combinations of diffusion process intervals and walk probabilities, were simultaneously and correctly simulated.

\subsubsection{Reactions in volume and surface compartments are verified}

For the irreversible reaction $A+B \stackrel{k_{A B}}{\longrightarrow} C$, it can be seen in Figure $2.4 A$ and $2.4 \mathrm{~B}$ that the survival probabilities of $A$ from simulations are in excellent agreement with the predicted theoretical curves in both type of compartments. The values of $D_{A}, D_{B}$ and $p_{j}$ are chosen such that Spatiocyte is evaluated for reactions that are minimally to maximally 


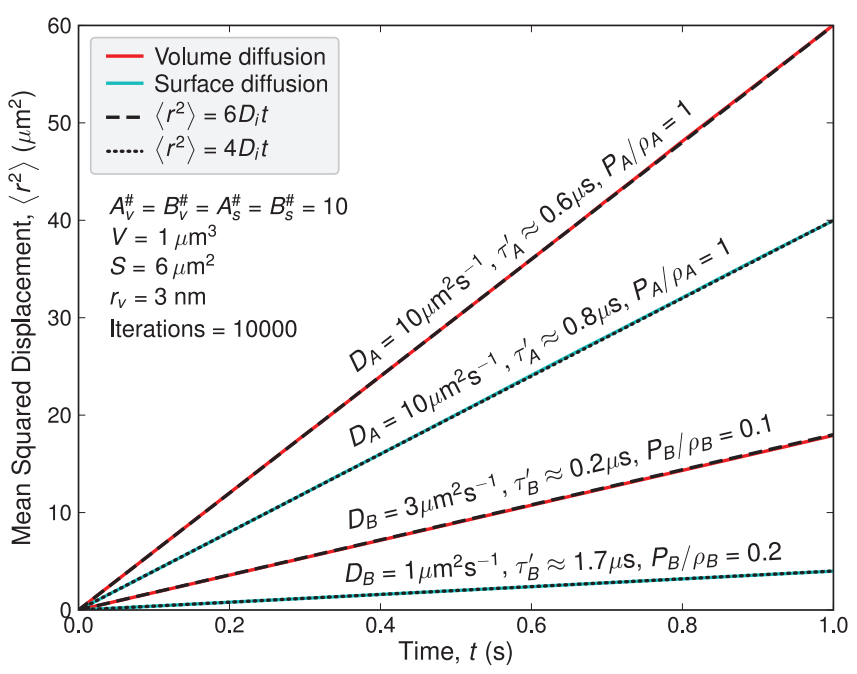

Figure 2.3: Verification of diffusion in volume and surface compartments. Solid lines show the mean squared displacement of molecules diffused according to (2.25) using the indicated parameters and averaged over 10000 runs with independent seeds. The lines almost perfectly overlap the predicted volume (dashed lines) and surface (dotted lines) diffusion results from analytic solutions. At the beginning of each run, molecules are placed randomly in their respective compartment. A cubic volume with periodic boundaries constitutes the volume compartment, while its six faces, covered with lipid molecules and imposed with periodic boundary condition at the edges, form the surface compartment. When a molecule walks over a periodic boundary, its absolute coordinate is updated to emulate borderless volume and surface compartments. All four species, with unique combinations of diffusion process interval $\tau_{i}^{\prime}$ and walk probability $\mathrm{P}_{i} / \rho_{i}$, were simultaneously simulated to also verify the correctness of the multi-timescale algorithm. In both compartments, the excluded volume effect by the molecules is negligible because they occupy less than $0.01 \%$ of the simulated volume and surface area.

diffusion-limited. In each compartment, the multi-timescale algorithm is also verified since the eight reactions were simultaneously performed correctly.

Compared to irreversible reactions, reversible reactions present a harder test to spatial modeling methods because newly dissociated molecules that are close to each other have a higher tendency to recombine. We performed the reversible reaction $A+B \underset{k_{C}}{\stackrel{k_{A B}}{\rightleftharpoons}} C$ with both weak $\left(k_{C}=5\right)$ 
and strong $\left(k_{C}=500\right)$ dissociation constants to evaluate the method. Since the dissociation reactions are diffusion-independent, they were ex-
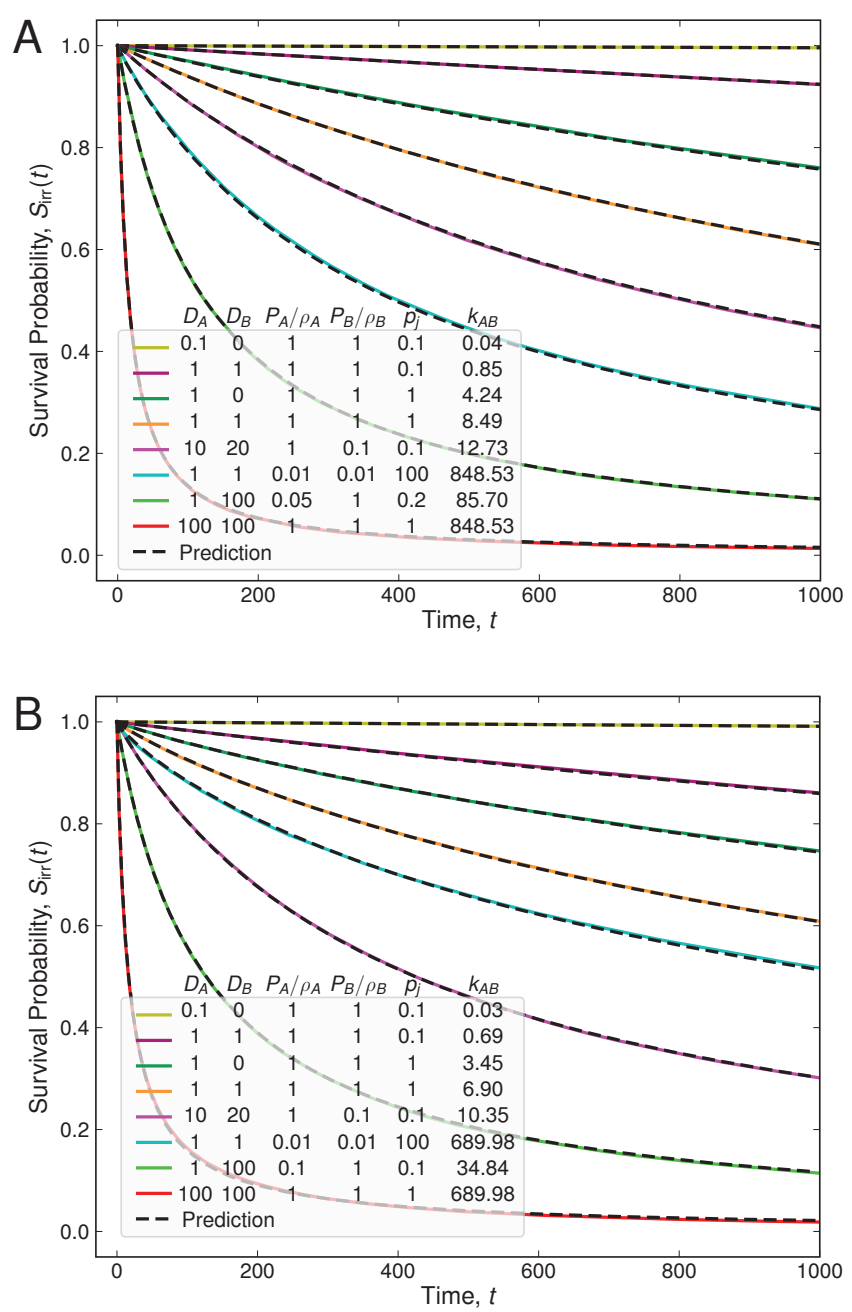

Figure 2.4: Verification of irreversible reactions in volume and surface compartments. The survival probability of $A$ in the second-order irreversible reaction $A+B \stackrel{k_{A B}}{\longrightarrow} C$, taking place in volume (A) and surface (B) compartments is shown. Dashed curves are the predicted survival probability according to (2.26), while the solid colored curves are the simulation results averaged over 1000 independent iterations. In each compartment, eight reactions were simultaneously simulated with various combinations of diffusion coefficients, $\mathrm{D}_{\mathrm{A}}$ and $\mathrm{D}_{\mathrm{B}}$, and the reactive collision probability $p_{j}$, as indicated in the inset legend table. Each reaction involves $100 \mathrm{~A}$ and $100 \mathrm{~B}$ molecules, with the voxel radius $r_{v}=0.5$. For the volume and surface compartments, $\mathrm{V}=1 \times 10^{6}$ and $\mathrm{S}=3.5 \times 10^{5}$, respectively. 
ecuted using the adapted NR method. Figure $2.5 \mathrm{~A}$ and $2.5 \mathrm{~B}$ show the simulation results when the reactions take place in the volume and surface compartments, respectively. Taking into account the noise limits, the results are in good agreement with the best known approximation theory, MPK 1 in both compartments, thus verifying the diffusion-independent and diffusion-influenced reactions of Spatiocyte. In addition, as previously reported in (Ridgway et al., 2008), mass action fails to correctly reproduce the predicted survival probability of $A$ for volume reversible reactions (Figure 2.5A) because it assumes $\mathrm{A}, \mathrm{B}$ and $\mathrm{C}$ to be $\mathrm{HD}$ species. Figure $2.5 \mathrm{~B}$ shows that mass action is also unable to reproduce the expected curves for surface reversible reactions.

\subsubsection{Spatiocyte reproduces implications of molecular crowding}

We evaluated Spatiocyte to reproduce the effects of volume exclusion by first examining the diffusion behavior of molecules in a crowded volume compartment. We mimicked the crowded Escherichia coli cytoplasm by occupying $34 \%$ of the $1 \mu \mathrm{m}^{3}$ compartment volume (Zimmerman and Trach, 1991) with inert and immobile crowder molecules. Figure 2.6A shows the time-dependent diffusion coefficients of six species that were diffused in the compartment. Of the six species, one is an Escherichia coli division site control protein, MinD with the diffusion coefficient $61 \mu \mathrm{m}^{2} \mathrm{~s}^{-1}$, obtained by fluorescence correlation spectroscopy (FCS) in a dilute solution (Loose et al., 2008). The results indicate that the species undergo anomalous diffusion (i.e., sub-linear scaling of the mean-squared displacement over time) when the volume compartment is crowded. Corroborating the results of previous off-lattice simulations (Ridgway et al., 2008), the time-dependent diffusion coefficients reach equilibrium by $10^{-4} \mathrm{~s}$, at which time they have decreased by about a factor of two compared to dilute levels.

Using the same six species, we investigated the diffusion behavior in two different cases of crowded surface compartment. In the first case (Figure 

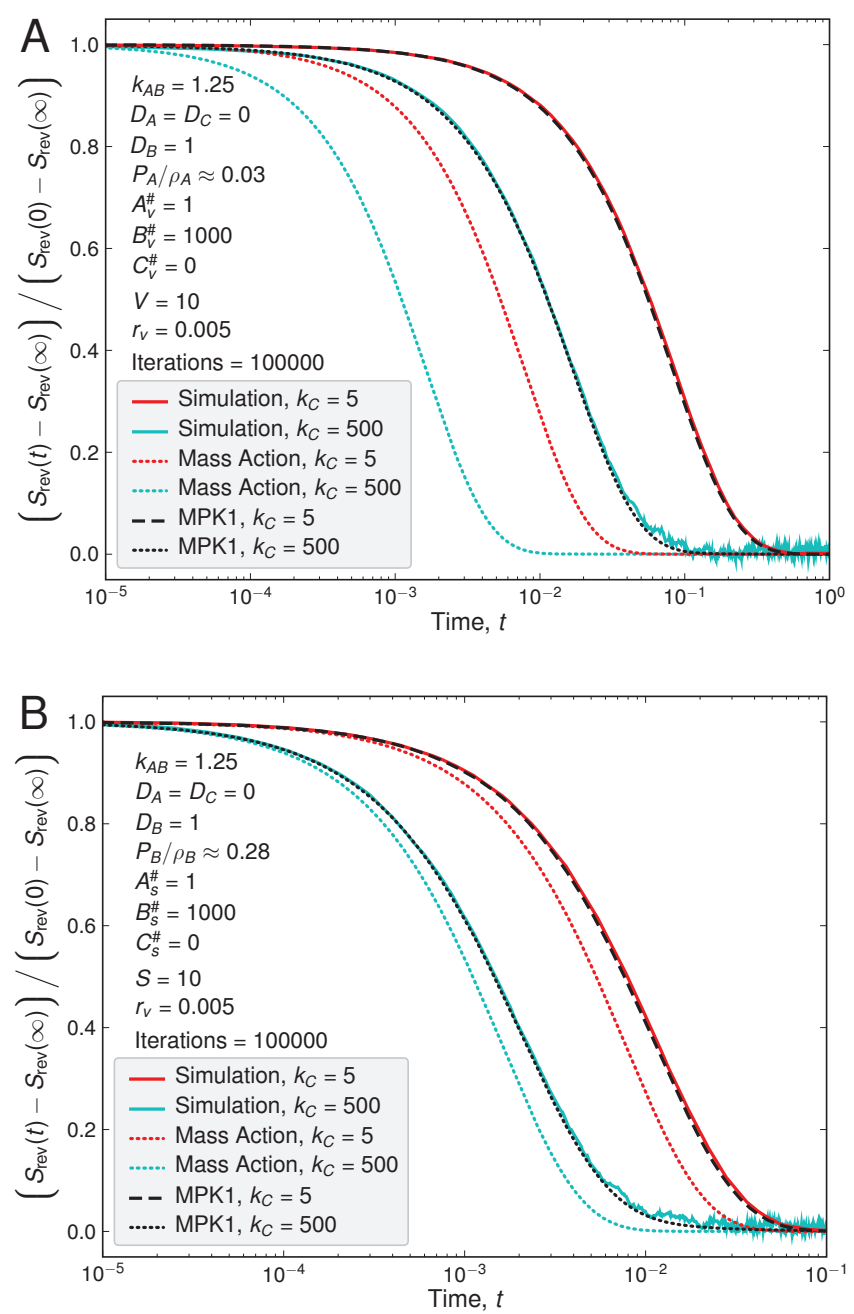

Figure 2.5: Verification of reversible reactions in volume and surface compartments. The normalized deviation of the survival probablity $S_{\text {rev }}(t)$ of $A$ from the predicted equilibrium value $S_{\text {rev }}(\infty)$ in the second-order reversible reaction $A+B \underset{k_{C}}{\stackrel{k_{A B}}{\rightleftharpoons}} C$, taking place in volume $(A)$ and surface (B) compartments is shown. Black dashed and dotted curves are the predicted values calculated according to MPK 1 theory (Sung and Lee, 1999; Popov and Agmon, 2001) in equation (2.31), dotted colored curves are theoretical predictions computed according to mass action, while the solid colored lines are the simulation results. The reaction and simulation parameters are indicated in the respective panels. In all reactions, the impact of molecular crowding is negligible because the molecules occupy less than $0.01 \%$ of the simulated volume and $0.8 \%$ of the surface area.

2.6B), we occupied $34 \%$ of the surface area with crowders to allow direct comparison to the previously observed behavior in volume compartment. 
In the second case (Figure 2.6C), 23\% of the surface area was occupied by the crowders to reflect the recently determined in vivo value (Dupuy and Engelman, 2008). The results of the first case show that the anomalous diffusion of the surface molecules is more pronounced than the observed behavior in the volume compartment. The elevated impact of crowding in the surface compartment is because of the increased obstructions to diffusion, resulting from the smaller dimensionality of the surface space (Zhou, 2009). Interestingly in the second case, although the area occupancy
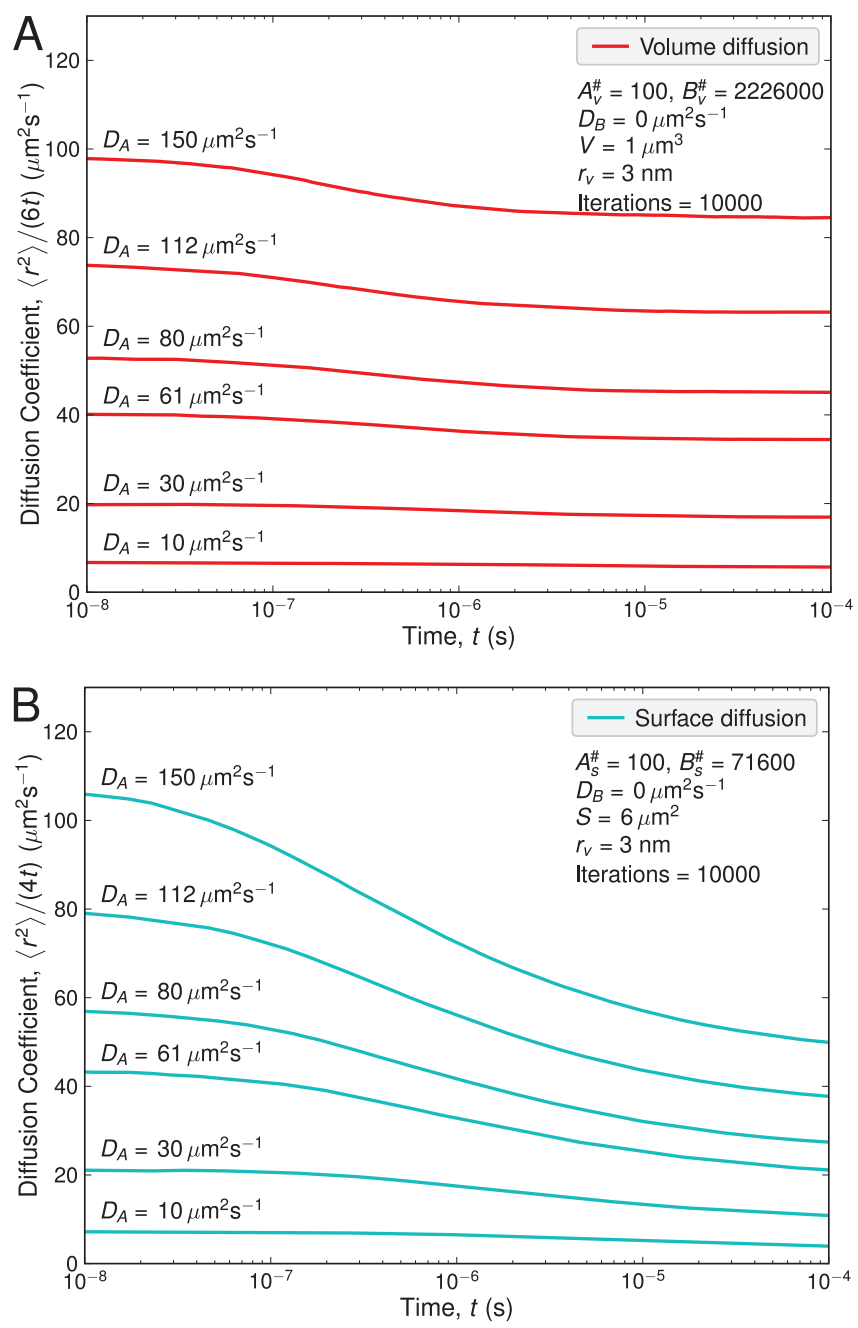

Figure 2.6: Implications of molecular crowding in diffusion and reaction processes. 

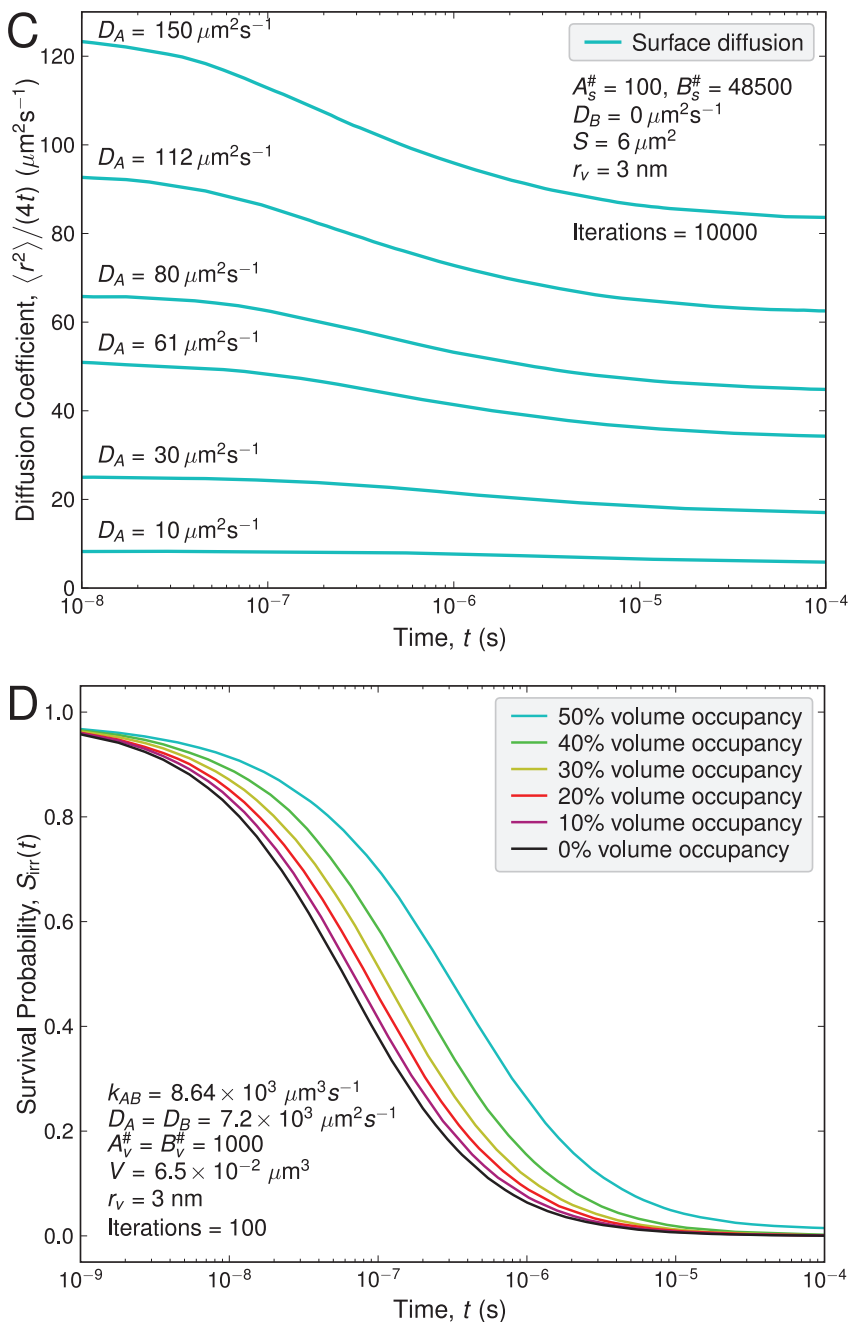

Figure 2.6: Implications of molecular crowding in diffusion and reaction processes (cont.). (A-C) The time-dependent diffusion coefficients at $34 \%$ occupied volume (A), 34\% occupied surface area (B) and $23 \%$ occupied surface area $(C)$. The diffusion coefficients are calculated from the equation $D(t)=\left\langle r^{2}\right\rangle /(2 l t)$, where $\left\langle r^{2}\right\rangle$ is the mean squared displacement and $l$ is the spatial dimensionality. Line labels show the diffusion coefficients of six simulated species at dilute levels. (D) The survival probability of $A$ in the volume irreversible reaction $A+B \stackrel{k_{A B}}{\longrightarrow} C$ at various occupied volume fractions (rounded to integer percentage). All simulation parameters are as indicated in the respective panels.

is $23 \%$, the surface diffusion coefficient at equilibrium $\left(t=10^{-4} \mathrm{~s}\right)$ is almost equivalent to that of the volume at $34 \%$ occupancy. 
We evaluated the effects of molecular crowding in volume irreversible reactions. Figure $2.6 \mathrm{D}$ shows that the apparent survival probability of the reacting molecules increases in line with the volume occupancy. There are more surviving reactants at higher occupancy because their timedependent diffusion coefficient is reduced by the increased hindrances from crowders. Together, these results confirm that our method can account for some of the important implications of molecular crowding in volume and surface compartments.

\subsubsection{Spatiocyte visualization method simulates microscopy images}

Figure 2.7 compares the localization patterns of simulated molecules at single molecule resolution to the corresponding simulated fluorescence microscopy image using the Spatiocyte visualization method. The radius of the molecules, occupying an Escherichia coli membrane, is $10 \mathrm{~nm}$ while the exposure time of the simulated image is $500 \mathrm{~ms}$. Since the molecules are very small, it is difficult to identify the patterns at single molecule resolution. Conversely, the patterns are clearly defined in the simulated fluorescence image because the overall trajectory of molecules during the exposure time is shown. Co-localizing molecules can also be identified by their blended colors.

Comparisons of actual microscopy images to the corresponding simulated images are shown in Figure 4.1C. The left column of the panel illustrates the in vivo localization patterns of MinD and MinE from the doubly labeled experiments by Shih et al. (2002). Using an exposure time of $500 \mathrm{~ms}$, the Spatiocyte visualization method closely reproduces the microscopy images in both individual and overlay panels. However, the simulated patterns are not as smooth as in the microscopy images possibly because the diffraction of light was not considered. It is also evident that the visualizations of single molecules do not clearly show the localization patterns as observed in the microscopy images. 

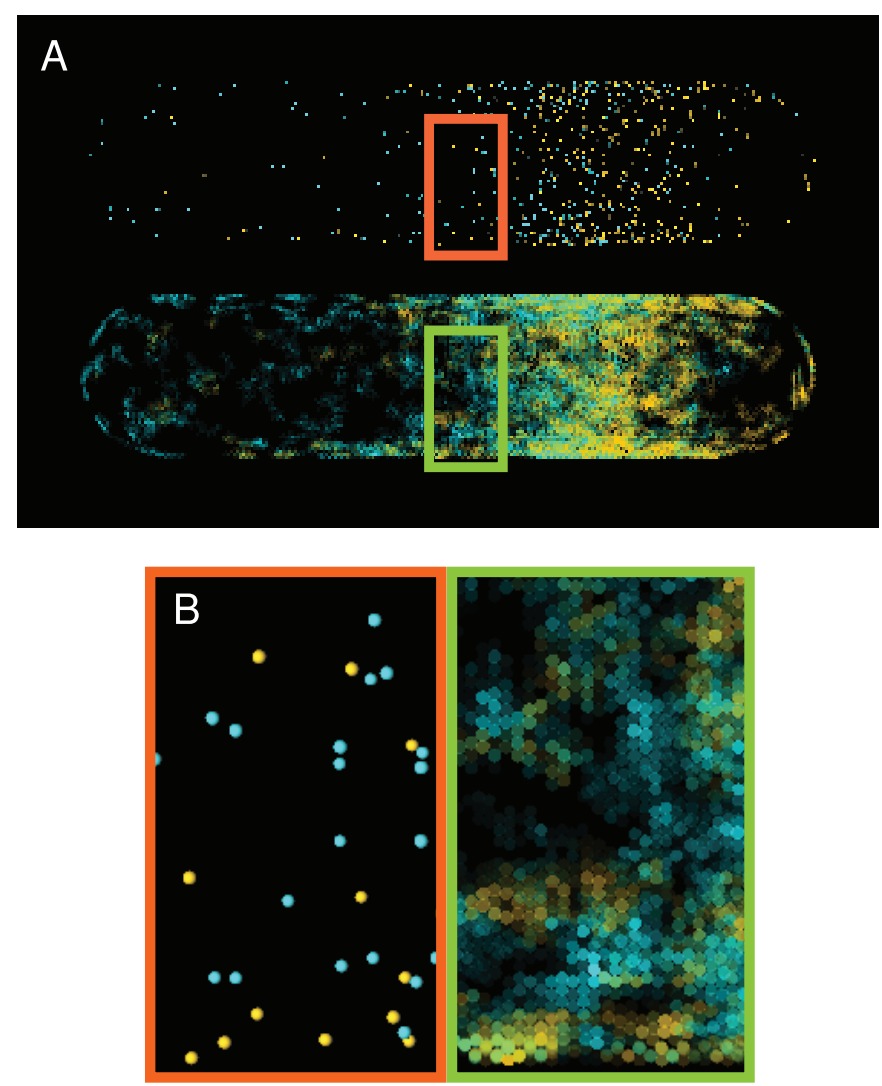

Figure 2.7: Comparison of single molecule visualization to simulated microscopy image. (A) Single molecule visualization (Top) of MinE.MinD $\mathrm{ATP}_{\mathrm{AT}}^{\mathrm{m}}$ (yellow) and $\mathrm{MinE}^{\mathrm{m}}$ (cyan) on the Escherichia coli membrane compared to the corresponding simulated fluorescence microscopy image (Bottom). The diffusion coefficients of the proteins are listed in Table 4.1. The exposure time of the fluorescence image is $500 \mathrm{~ms}$. (B) The magnified area of the respective boxes shown in (A).

\subsection{DISCUSSION}

In this chapter, we developed and verified a new lattice-based method, Spatiocyte that can model surface and volume intercompartmental RD processes, while also being able to account for the implications of stochasticity and molecular crowding arising from the physical dimensions of molecules. To our knowledge, there is currently no single method that can do this (see Table 1.1). We have also adopted several optimization strategies to reduce the high computational cost associated with spatial modeling 
methods. Firstly, unlike other methods, Spatiocyte can simultaneously simulate highly concentrated or HD species at compartmental scale and low copy or heterogeneously distributed species at molecular scale. This allows the method to skip diffusing each molecule belonging to the highly concentrated species, which would incur very high computational cost. Molecules within the two distinct spatial scales can react as usual with each other. Secondly, the reactions involving HD species are event-driven so that computations are performed only when necessary, thus allowing bigger jumps in the simulation time. Thirdly, to support fast RD simulation in crowded compartments, the simulation space is discretized into hexagonal close-packed lattice that enables rapid resolving of molecular collisions.

The obvious limitation of Spatiocyte is that the non-HD molecules have to be almost similar in size to occupy the voxels. This presents a problem when modeling molecules with disparate densities in a crowded compartment since the size of the molecules directly influences RD. Nonetheless, in most spatial modeling applications, the molecules are assumed to be uniform sized. In addition, the method can also be extended to model molecules of different sizes by occupying multiple voxels with a single molecule according to its size. In this case, the reactive collision probability will need to be readjusted to account for the collisions that take place at the multiple voxels occupied by the molecule.

We also developed a visualization method that uses the temporal positional data of simulated molecules to simulate optical microscopy images of fluorescent labeled proteins. It does this by showing the trajectory of simulated molecules averaged according to the camera exposure time used in the microphotography process. The method enabled us to directly match our simulation results with the localization patterns of MinD and MinE in Escherichia coli and make objective evaluations. Schaub et al. (2007) have also developed a method to simulate the microscopy images but it is intended for almost immobile, non-diffusing structures, such as actin fila- 
ments, and cannot be directly applied for diffusing molecules as required by our method.

In our results, the $34 \%$ crowded volume compartment caused the diffusion coefficient of MinD to be reduced by a factor of two to approximately $37 \mu \mathrm{m}^{2} \mathrm{~s}^{-1}$ from its dilute level of $61 \mu \mathrm{m}^{2} \mathrm{~s}^{-1}$ (Loose et al., 2008). However, the measured diffusion coefficient of MinD in the $34 \%$ crowded Escherichia coli cytoplasm is only $16 \mu \mathrm{m}^{2} \mathrm{~s}^{-1}$ (Meacci et al., 2006). The additional twofold reduction can be attributed to the several factors listed by Ridgway et al. (2008), which include the intracellular viscosity and the nonspecific binding to other molecules in the cytoplasm. This suggests that adding crowder molecules alone may not be sufficient to reproduce the cytoplasmic environment in a simulated model.

Our results also support the suggestion by Loose et al. (2008) that molecular crowding on the cell membrane decreases the lateral diffusion coefficient of MinD compared to that of in vitro. Additional reduction in the diffusion coefficients could also be attributed to the protein oligomerization and polymerization-depolymerization kinetics on the membrane. Polymers on the membrane may also present as bigger hindrances to the mobility of the proteins. 
IMPLEMENTATION OF SPATIOCYTE WITH THE E-CELL SYSTEM

The E-Cell System is one of the well known and advanced open-source simulation platforms to model and analyze both small- and large-scale biochemical reaction networks in living cells (Tomita et al., 1999). The driver algorithm of the E-Cell System (version 3) supports concurrent executions of multiple simulation algorithms, whose time steps are independently advanced in continuous-time, discrete-time or discrete-event manner at varying timescales (Takahashi et al., 2004). Multiple sessions of simulations, usually required for estimation of reaction parameters and to obtain the averaged results from stochastic reactions, can be simultaneously executed with its distributed computing utility (Sugimoto et al., 2005; Sugimoto, 2009). Simulation runs can be automated and modified ex tempore with Python scripting, while new simulation algorithms can be developed using $\mathrm{C}++$ and incorporated into the system as plug in modules.

Recent advances in molecular biology suggest that modeling reaction networks alone is not sufficient to accurately reproduce certain important cellular processes such as cell division (Lutkenhaus, 2007) and gene expression (Talbert and Henikoff, 2006). In Chapter 1 we have reviewed how the physical location, crowding and diffusion of molecules in cell compartments play crucial roles in these processes.

In the rod-shaped Escherichia coli, the division site is restricted to the midcell by nucleoid occlusion and the pole-to-pole oscillation of the proteins MinC, MinD and MinE, collectively called the MinCDE system (reviewed by Lutkenhaus (2007)). The periodic oscillation is established because of intricately controlled reaction and diffusion of the proteins in the cytoplasm and the inner membrane. A simplified model of the system is illustrated in Figure 3.1, which includes only MinD and MinE since MinC is not necessary for the oscillation. Division at the midpoint of the cell is impor- 
tant to ensure equal distribution of cell contents to the two daughter cells. The MinCDE molecules, found in low copies in the cytoplasm and on the inner cell membrane compartments, are not evenly distributed temporally. As a result, the rate of reactions, which is determined by the frequency of collision between reacting molecules, is influenced by diffusion and physical localization within the compartments. The molecules undergo surface and volume reaction-diffusion (RD) on the cell membrane and in the cytoplasm respectively.

Current algorithm modules of the E-Cell System assume that reactions take place between molecules that are uniformly distributed within the reaction compartment. It is also not possible to specify the physical location of each molecule. In this chapter, we describe the extension of the ECell System to model the spatial localization and RD of molecules by implementing the Spatiocyte method described in Chapter 2. Out of the several available methods (see Table 1.1), ours is the only one that can account for the important implications of volume exclusion by molecules and RD in both volume and surface spaces. To evaluate the new approach, we compare the simulation results of the MinDE system with the results obtained from experiments and previous computational work.

\subsection{METHODS}

In this section, we briefly recall the proposed RD scheme in Chapter 2 before providing the details of the implementation with the E-Cell System.

\subsubsection{The reaction-diffusion scheme}

Molecules diffuse freely in space by making random walks (Berg, 1993). According to the Collins and Kimball theory (Collins and Kimball, 1949), when two reactive molecules come into contact (i.e., collide), they react with a certain probability $p$, which is related to the reaction rate constant $k$. To avoid molecule search when checking for collisions, we have discretized the 


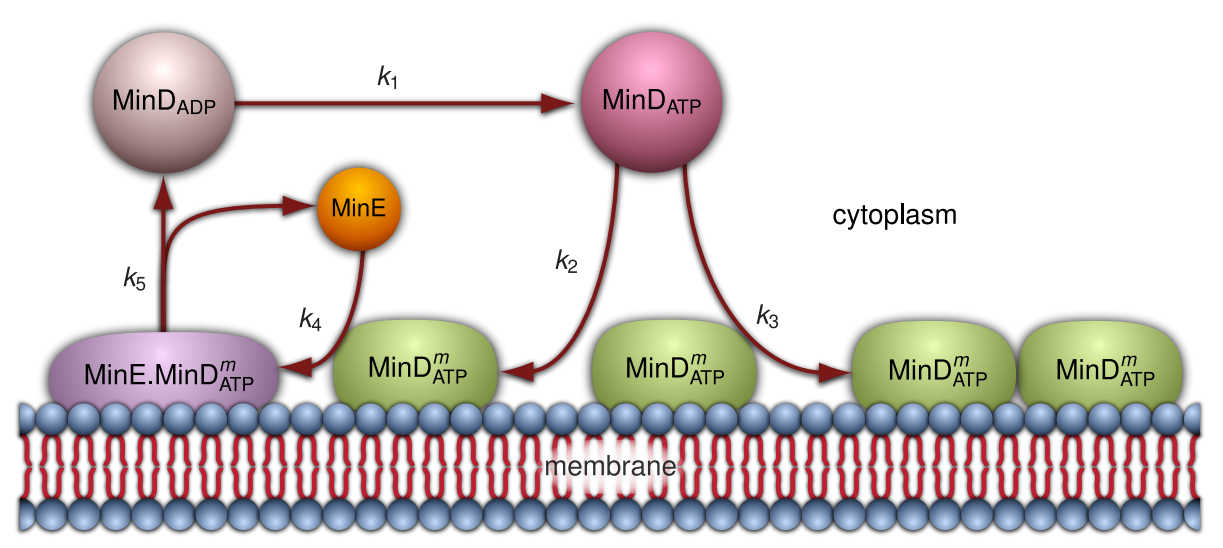

Figure 3.1: The simplified oscillation model of the MinDE sytem in Escherichia coli. This model is adapted from (Huang et al., 2003). MinC is not represented because it is not essential for the oscillation. Arrows depict the five basic reactions in the model. In the first reaction, $\mathrm{MinD}_{\mathrm{ADP}}$ exchanges nucleotide to become $\mathrm{MinD}_{\mathrm{ATP}}$ with the rate $\mathrm{k}_{1}$. In the form of $\mathrm{MinD}_{\mathrm{ATP}}$, the molecule can bind to the membrane either autonomously with the rate $k_{2}$ or cooperatively with a another membrane-bound $\operatorname{MinD}_{\mathrm{ATP}}^{\mathrm{m}}$ at the rate $k_{3}$. Cytosolic MinE is also recruited to the membrane by $\operatorname{Min}_{\mathrm{ATP}}^{\mathrm{m}}$ with the rate $k_{4}$ to form MinE.MinD $D_{A T P}^{m}$. The ATPase function of MinD is activated by MinE in the MinE.MinD $\mathrm{ATP}_{\mathrm{m}}^{\mathrm{m}}$ complex and consequently, $\operatorname{Min}_{\mathrm{ATP}}$ is converted to $\mathrm{MinD}_{\mathrm{ADP}}$ that cannot stay bound to the membrane. This is represented by the fifth reaction, in which the MinE.MinD $D_{\text {ATP }}^{m}$ complex dissociates from the membrane at the rate $k_{5}$ and forms the cytosolic monomers MinE and MinD $\mathrm{ADP}$.

space into hexagonal close-packed lattice (Conway and Sloane, 1998). Each sphere voxel in the lattice has 12 adjoining neighbor voxels. To account for volume exclusion and molecular crowding, each voxel can be occupied by a single molecule. The radius of the voxels is set according to the size of diffusing molecules. A molecule can walk to a randomly selected neighbor voxel in an interval $\tau_{d}$ following the Einstein-Smoluchowski expression for diffusion

$$
\tau_{d}=\frac{\left\langle r^{2}\right\rangle}{2 l D}
$$


where $\left\langle\mathrm{r}^{2}\right\rangle$ and $\mathrm{D}$ are the mean squared displacement and the diffusion coefficient of the molecule respectively, and $l=2$ for surface diffusion while for volume diffusion, $l=3$. Since in the interval $\tau_{d}$ the molecule walks to a neighbor voxel, the mean squared displacement is given by the lattice spacing. We have derived the spacing for surface and volume diffusion, and the connection between $p$ and $k$ in Chapter 2. At the destination voxel, the walking molecule may collide with another molecule that is a reactant pair and react if an independent random number drawn from a unit uniform distribution is less than $p$.

\subsubsection{E-Cell System data structure and driver algorithm}

The specific details of the E-Cell System data structure, driver and integration algorithms have previously been described (Takahashi et al., 2004). We briefly provide the data structure and the driver algorithm here to characterize the implementation of our algorithm modules. We adopt the notations in (Takahashi et al., 2004) and capitalize the class names.

A reaction network system is represented in E-Cell as a Model, specified by the user in E-Cell Model description language (EML), a subset of XML. Figure 3.2 shows the data structure of the Model class, which contains a list of state Variables and Steppers. The Stepper class is the main algorithm module of the Model and it operates with a set of Processes, the current local time $\tau$ and the step interval $\Delta \tau$, a step method that advances the Stepper in time either in a continuous-time, discrete-time or discrete-event fashion, and an interruption method that allows other Steppers to notify the Stepper when they modify a read Variable of the Stepper. The Process is a lower level algorithm module that directly reads or modifies the state Variables according to the algorithm using a transition-function. Here, the instances of the state Variables are accessed by dereferencing the Variable References. 


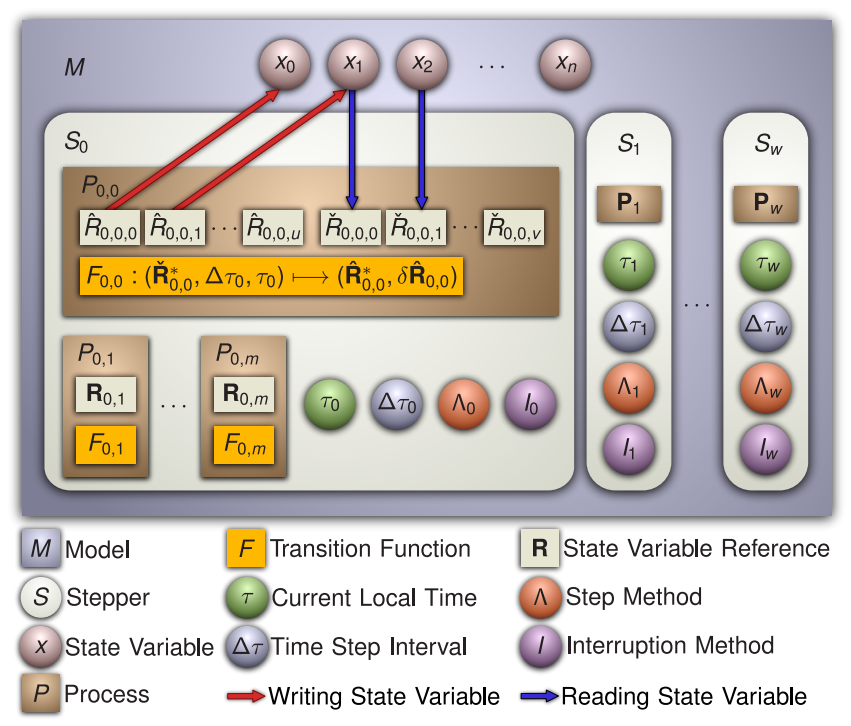

Figure 3.2: The data structure of the E-Cell Model.

Central to the E-Cell driver algorithm is a priority queue that arranges the Steppers according to the scheduled time of execution, given as $\tau+\Delta \tau$. At initialization, the global time $t$ and the local time $\tau$ of Steppers are reset. Next, the step method of the Stepper $S_{i}$ with the minimum scheduled time is called and the global time is updated to $t=\tau_{i}+\Delta \tau_{i}$. The step method also sets the local time to $\tau_{i}=\tau_{i}+\Delta \tau_{i}$ and calls the transition function of its Processes to update the state Variables. The method may also update the next step size $\Delta \tau_{i}$ and call the interruption method of other Steppers whose read Variable has been modified. The Stepper $S_{i}$ is rescheduled in the priority queue according to the new scheduled time. The same procedure is repeated for the Stepper with the next earliest scheduled time in the priority queue until the simulation is ended.

\subsubsection{Implementation of reaction-diffusion with E-Cell}

We have implemented the proposed reaction-diffusion scheme using the E-Cell System by creating two basic algorithm modules - a Diffusion Process and a Reaction Process. The molecules are represented as the state Variables of the Model. For each diffusion coefficient in simulation, a Diffusion Process 
object is created to walk the molecules. Likewise, a Reaction Process object is instantiated for every reaction involving a diffusing molecule. A discreteevent Stepper advances the Diffusion Process in time and handles the lattice structure and the physical location of molecules. The transition function of the Diffusion Process walks each molecule to a randomly selected adjoining voxel in a diffusion step interval. When a molecule collides with a reactant pair, the transition function of the corresponding Reaction Process is called. If the reactive collision probability is met, the Process removes the collided molecules and replaces them with one or two product molecules, as specified by the reaction.

\subsection{APPLiCATION RESULtS}

We modeled the oscillatory behavior of molecules in the MinDE system to validate our approach because it involves both surface and volume RD, and spatiotemporal localization of molecules. We describe the computational model of the system before presenting the results of simulation.

\subsubsection{The MinDE Model}

In Escherichia coli, the FtsZ membrane protein initiates cell division physically by polymerizing and constricting annularly at the middle of the long axis of the rod-shaped cell (Lutkenhaus, 2007; Löwe and Amos, 2009). Although the protein can diffuse over the entire membrane, nucleoid occlusion prevents the polymerization from taking place over the nucleoid mass, leaving only the midcell and the two cell poles as viable locations for polymerization (Woldringh et al., 1990, 1991; Bernhardt and de Boer, 2005). Nonetheless, because of the inhibition by MinC proteins at the poles $(\mathrm{Hu}$ et al., 1999; $\mathrm{Hu}$ and Lutkenhaus, 1999), the polymerization can only take place at the midcell. During the cell cycle, MinD with the help of MinE forms polar zones which oscillate from one pole to the other. Since MinC 
piggybacks on MinD and the oscillation occurs on the rod-shaped cell with some dwelling time at the poles, the time-averaged concentration of MinC at the middle of the cell is kept low, permitting FtsZ to polymerize.

According to the model by Huang et al. (2003) (illustrated in Figure 3.1), cytosolic MinD in the ATP-bound form $\left(\mathrm{MinD}_{\mathrm{ATP}}\right)$ binds to the membrane either cooperatively with another membrane bound $\operatorname{MinD}\left(\operatorname{MinD}_{\mathrm{ATP}}^{\mathrm{m}}\right)$ or independently. MinE from the cytoplasm inhibits $\mathrm{MinD}_{\mathrm{ATP}}^{\mathrm{m}}$ by first associating with it and setting off the ATPase function of MinD that hydrolyzes the bound ATP to ADP. The membrane-bound MinE and the ADP-bound form of MinD (MinD $\left.\mathrm{ADP}_{\mathrm{AP}}\right)$ then dissociate to the cytoplasm. In the cytoplasm, MinD $D_{\mathrm{ADP}}$ is phosphorylated and takes the form of $\mathrm{MinD}_{\text {ATP }}$ again. MinC is not explicitly represented in the model because it is usually attached to MinD and experimental data indicate that the oscillation can take place without it (Hu and Lutkenhaus, 1999; Raskin and de Boer, 1999). The series of reactions in the model are as follows:

$$
\begin{gathered}
\operatorname{MinD}_{\text {ADP }} \stackrel{k_{1}}{\longrightarrow} \operatorname{MinD}_{\text {ATP }} \\
\operatorname{MinD}_{\text {ATP }} \stackrel{k_{2}}{\longrightarrow} \operatorname{MinD}_{\text {ATP }}^{m} \\
\operatorname{MinD}_{\text {ATP }}+\operatorname{MinD}_{\text {ATP }}^{m} \stackrel{k_{3}}{\longrightarrow} 2 \operatorname{MinD}_{\text {ATP }}^{m} \\
\text { MinE }+\operatorname{MinD}_{\text {ATP }}^{m} \stackrel{k_{4}}{\longrightarrow} \operatorname{MinE} \cdot M_{\text {MinD }}^{m} \\
\text { MinP } \\
\text { MinE.MinD ATP }
\end{gathered}
$$

\subsubsection{Simulation Results}

To simulate the MinDE oscillation model using the proposed approach, we applied the parameters from (Fange and Elf, 2006) (listed in Table 3.1). However, we reduced the radius of our voxels to more closely reflect the size of diffusing molecules. Another variation between our method and 
the MesoRD method in (Fange and Elf, 2006) is that our molecules can exhibit excluded volume.

Figure 3.3 shows the random distribution of cytosolic $\operatorname{MinD}_{\mathrm{ADP}}, \mathrm{MinD}_{\mathrm{ATP}}$ and MinE molecules at initialization. The pole-to-pole oscillation of membranebound MinD $\mathrm{ATP}^{\mathrm{m}}$, as shown in Figure 3.4, is spontaneously triggered after about 1 minute of simulated time although all molecules were initially randomly distributed in the cytoplasm. For the 1 minute of simulated time, it takes about 14 minutes of simulation on an Intel Core 2 Extreme $3.2 \mathrm{GHz}$ system with 8 GB of RAM. The oscillation has an average period of $36 \mathrm{sec}-$ onds which corresponds to what has been observed experimentally (Hale et al., 2001; Raskin and de Boer, 1999). The period is also in close agreement with the value from the previously reported computational model (Fange and Elf, 2006). Consistent with the observations by Huang et al. (2003) and Fange and Elf (2006), the period increased proportionally to the number of MinD, while reduced proportionally to the amount of MinE in the model. In addition, as observed in the MinDE localization studies in Escherichia coli (Shih et al., 2002), the membrane-bound MinE.MinD $D_{\text {ATP }}^{m}$ dimers appear to be lagging behind $\mathrm{MinD}_{\mathrm{ATP}}^{\mathrm{m}}$ molecules when they migrate from one pole to the other. Taken together, our simulations closely reproduce the results from both experimental and previous computational studies.

Here, we describe the steps that trigger the spontaneous oscillation. Initially, all molecules are evenly distributed in the cytoplasm. Very small number of $\mathrm{MinD}_{\mathrm{ATP}}$ molecules begin to independently associate at random locations on the membrane and rigorously recruit other $\mathrm{MinD}_{\mathrm{ATP}}$ molecules cooperatively. As shown in Figure 3.5, the recruitment gives the appearance of growing patches on the membrane. Cytosolic MinE molecules are attracted to these patches because of their high affinity to $\mathrm{MinD}_{\mathrm{ATP}}^{\mathrm{m}}$ and form MinE.MinD $D_{\mathrm{ATP}}^{\mathrm{m}}$ dimers. Soon the patches loosely cover the entire membrane because the rate of MinD recruitment is faster than the rate of dissociation, even after almost all MinE are found in the MinE.MinD $\mathrm{ATP}_{\mathrm{m}}^{\mathrm{m}}$ dimer form on the membrane. At random locations on the 


\section{Table 3.1: Parameters of the simple MinDE model.}

\begin{tabular}{ll}
\hline Parameter & Value \\
\hline$k_{1}$ & $0.5 \mathrm{~s}^{-1}$ \\
$k_{2}$ & $0.0125 \mu \mathrm{ms}^{-1}$ \\
$k_{3}$ & $0.0149 \mu \mathrm{m}^{3} \mathrm{~s}^{-1}$ \\
$k_{4}$ & $0.0923 \mu \mathrm{m}^{3} \mathrm{~s}^{-1}$ \\
$k_{5}$ & $0.7 \mathrm{~s}^{-1}$ \\
$\mathrm{D}_{\text {cytoplasm }}$ & $2.5 \mu \mathrm{m}^{2} \mathrm{~s}^{-1}$ \\
$\mathrm{D}_{\text {membrane }}$ & $0.01 \mu \mathrm{m}^{2} \mathrm{~s}^{-1}$ \\
Cell volume & $3.27 \mu \mathrm{m}^{3}$ \\
Cell radius & $0.5 \mu \mathrm{m}$ \\
Voxel radius & $8 \mathrm{~nm}$ \\
Initial MinD & 2001 \\
Initial MinD & molecules \\
Initial MinE molecules & 2001 \\
\end{tabular}

membrane, some $\mathrm{MinD}_{\mathrm{ATP}}^{\mathrm{m}}$ patches are free from MinE inhibition because of the limited cytosolic MinE molecules. In addition, these patches also become more persistent at locations farther from the dissociating patches, where they are less inhibited by the newly released MinE molecules and where $\operatorname{MinD}_{\text {ATP }}$ can escape cooperative recruitment by $\operatorname{MinD}_{\mathrm{ATP}}^{\mathrm{m}}$ in the dissociating patches. Finally, patches (or polar zones) form alternately (i.e., oscillate) at the two poles of the cell because the poles are sufficiently far from each other to avoid both the rapid inhibition by the released MinE and the cooperative recruitment by $\mathrm{MinD}_{\mathrm{ATP}}^{\mathrm{m}}$. During the oscillation cycle, the MinE.MinD $\mathrm{ATP}_{\mathrm{m}}^{\mathrm{m}}$ dimers appear to be lagging behind $\mathrm{MinD}_{\mathrm{ATP}}^{\mathrm{m}}$ because the released MinE molecules from the opposite pole find $\operatorname{MinD}_{\mathrm{ATP}}^{\mathrm{m}}$ at the rim of the polar zone first.

From the simulations, we observed several important features of the MinDE system that support the periodic oscillations. When we increased the nucleotide exchange rate of $\operatorname{MinD}_{\mathrm{ADP}}$ to $k_{1}=1 \mathrm{~s}^{-1}$, the population of $\mathrm{MinD}_{\mathrm{ATP}}$ increased, resulting in more $\mathrm{MinD}_{\mathrm{ATP}}^{\mathrm{m}}$ and longer polar zones. Since there are more $\mathrm{MinD}_{\mathrm{ATP}}^{\mathrm{m}}$ to be activated by MinE, the oscillation 


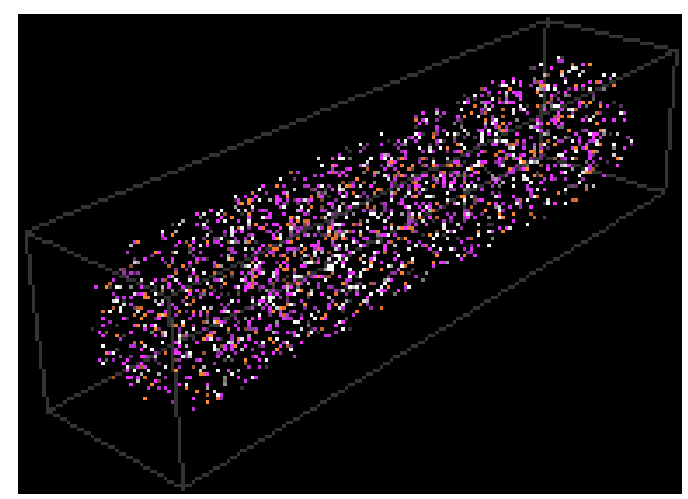

Figure 3.3: Simulated cytosolic molecules of the MinDE system. $\operatorname{MinD}_{\mathrm{ATP}}$ (purple), $\mathrm{MinD}_{\mathrm{ADP}}$ (white) and MinE (red) are randomly distributed in the cytoplasm of Escherichia coli at initialization.

period also increased to about 63 s. Conversely, the duration of an oscillation cycle is reduced to approximately $20 \mathrm{~s}$ when the rate is decreased to $\mathrm{k}_{1}=0.3 \mathrm{~s}^{-1}$ because of the limited number of MinD $\mathrm{ATP}_{\text {copies available. }}$

The reactive collision probability $p$ for $\mathrm{MinD}_{\mathrm{ATP}}$ to associate independently to the lipid molecules on the membrane $\left(k_{2}=0.0125 \mu \mathrm{ms}^{-1}, \mathrm{p}=\right.$ $\left.0.274 \times 10^{-4}\right)$ is about four orders of magnitude lower than to associate cooperatively with another membrane-bound $\operatorname{MinD}_{\text {ATP }}^{m}\left(k_{3}=0.0149 \mu \mathrm{m}^{3} \mathrm{~s}^{-1}, \mathrm{p}=\right.$ $0.16)$, even though the number of lipid molecules ( 72000$)$ is only about an order of magnitude more than that of $\mathrm{MinD}_{\mathrm{ATP}}^{\mathrm{m}}$ ( 1400). This ensures that MinD $_{\text {ATP }}$ only binds to the membrane independently to nucleate bindings

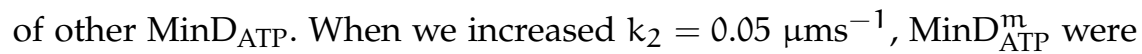
found loosely distributed in the polar zone that extended beyond the midcell because the enhanced nucleation rate allows $\operatorname{MinD}_{\text {ATP }}$ to successfully bind almost anywhere on the membrane. Occasionally, there were no clear definition of the polar zones, with MinD $\mathrm{ATP}_{\mathrm{AT}}^{\mathrm{m}}$ and MinE.MinD $\mathrm{ATP}_{\mathrm{ATP}}^{\mathrm{m}}$ covering the entire membrane. The oscillation period increased moderately to about $44 \mathrm{~s}$. Reducing $\mathrm{k}_{2}=0.0009 \mu \mathrm{ms}^{-1}$ shortened the period marginally to $33 \mathrm{~s}$ and displayed erratic nucleation patterns, with the polar zones frequently appearing and growing near the midcell. In addition, because of the decreased nucleation rate, cooperative membrane recruitments of $\operatorname{MinD}_{\text {ATP }}$ 
dominated further and rigorous membrane associations occurred at the nucleation sites.

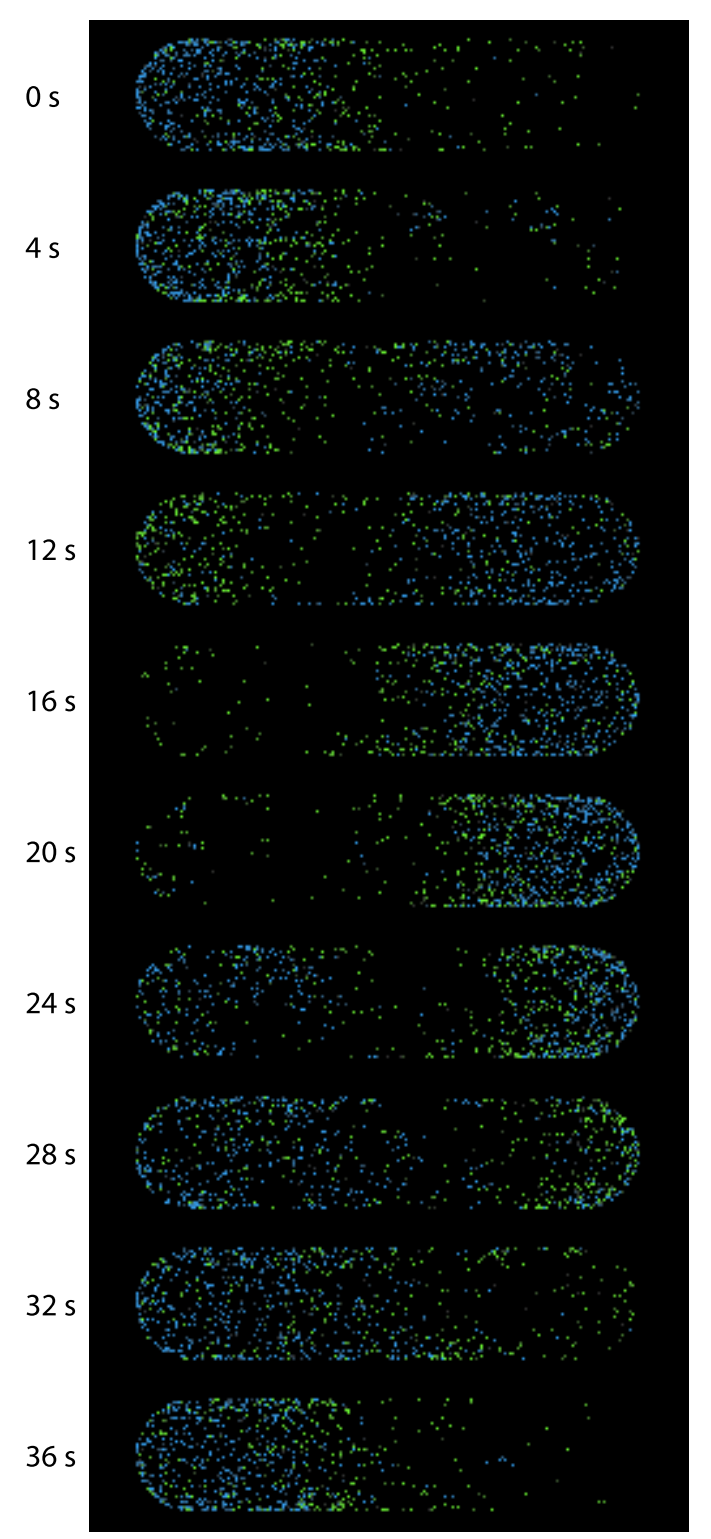

Figure 3.4: MinDE oscillations on the membrane of Escherichia coli. MinD $D_{\text {ATP }}^{m}$ monomers and MinE.MinD $D_{\text {ATP }}^{m}$ dimers are shown in cyan and green respectively. The $\mathrm{MinD}_{\mathrm{ATP}}^{\mathrm{m}}$ monomers appear to lead the dimers in the oscillation cycle which has an average period of 36 seconds.

When the rate of cooperative recruitment was increased to $k_{3}=0.034 \mu \mathrm{m}^{3} \mathrm{~s}^{-1}$, the oscillation was not triggered because MinE cannot rapidly activate the 
rigorously recruited $\mathrm{MinD}_{\mathrm{ATP}}^{\mathrm{m}}$. Therefore, both $\mathrm{MinD}_{\mathrm{ATP}}^{\mathrm{m}}$ and MinE.MinD $\mathrm{ATP}_{\mathrm{AT}}^{\mathrm{m}}$ were uniformly distributed on the membrane. Setting $k_{3}=0.024 \mu \mathrm{m}^{3} \mathrm{~s}^{-1}$ stimulated the oscillation but the polar zones were not clearly defined and extended well over the midcell because of the larger population of $\mathrm{MinD}_{\mathrm{ATP}}^{\mathrm{m}}$. The oscillation period also increased to approximately 50 s. Reducing $\mathrm{k}_{3}=0.004 \mu \mathrm{m}^{3} \mathrm{~s}^{-1}$ also prevented the oscillation because $\mathrm{MinD}_{\text {ATP }}$ cannot successfully bind to the membrane-MinE rapidly dissociates them since their cooperative recruitment activity has been weakened. However, setting $k_{3}=0.007 \mu \mathrm{m}^{3} \mathrm{~s}^{-1}$ started the oscillation with a reduced period of about $30 \mathrm{~s}$, while the polar zones were occasionally nucleated near the midcell.

Reducing or increasing the MinE membrane recruitment rate $k_{4}$ has generally the opposite effect of $k_{3}$. This is because the membrane associated MinE activates MinD ATPase function that dissociates MinD to the cytoplasm. Increasing the rate to $k_{4}=0.4 \mu^{3} \mathrm{~s}^{-1}$ generated the oscillation with a shortened period of about 30 s since more MinE.MinD $D_{\text {ATP }}^{m}$ are available to activate MinD. The polar zones were nucleated both near the midcell and at the poles, resulting in their rapid growth in each cycle. Conversely, setting $\mathrm{k}_{4}=0.05 \mu \mathrm{m}^{3} \mathrm{~s}^{-1}$ produced polar zones that are not clearly defined with an oscillation period of approximately $41 \mathrm{~s}$. Both polar zones frequently appeared simultaneously with one usually covering more than one half of the cell long axis.

The MinE.Min $D_{A T P}^{m}$ dissociation rate $k_{5}$ has almost the same properties as $k_{4}$ but with higher efficacy. When the rate was increased to $k_{5}=1.2 \mathrm{~s}^{-1}$, the polar zones were shorter and cycled between the poles with a period lasting approximately $12 \mathrm{~s}$. The higher rate increases the population of free MinE that can associate and activate other $\mathrm{MinD}_{\mathrm{ATP}}^{\mathrm{m}}$, thus reducing the size of the polar zones and increasing the oscillation speed. On the other hand when the rate was reduced to $k_{5}=0.5 \mathrm{~s}^{-1}$, the period increased to about $54 \mathrm{~s}$, while the polar zones were nucleated at the cell poles and extended beyond the midcell. 


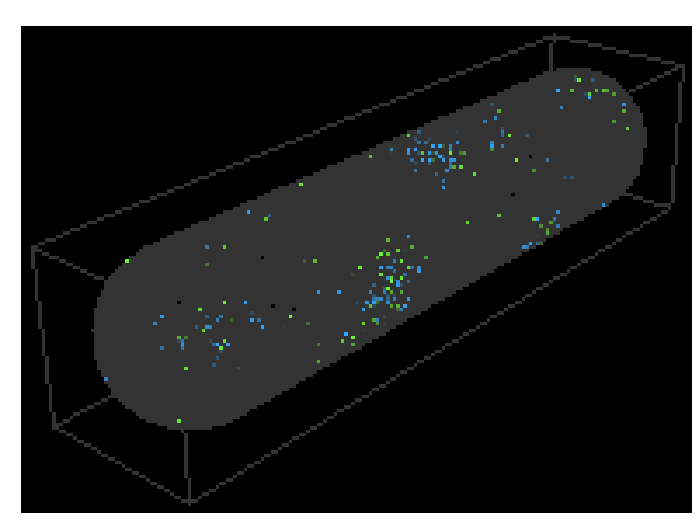

Figure 3.5: Initial patches of MinD (cyan) and MinE.MinD $\mathrm{ATP}_{\text {(green) }}$ forming on the membrane (gray).

The significantly slower diffusion coefficient of $\mathrm{MinD}_{\mathrm{ATP}}^{\mathrm{m}}$ and MinE.MinD $\mathrm{ATP}^{\mathrm{m}}$ on the membrane prohibits the molecules from rapidly achieving uniform concentration on the membrane. By increasing the diffusion coefficient twofold to $D_{\text {membrane }}=0.02 \mu \mathrm{m}^{2} \mathrm{~s}^{-1}$, the polar zones extended beyond the midcell and were not clearly defined. The oscillation period was about 6o s. Increasing the coefficient further to $D_{\text {membrane }}=0.05 \mu \mathrm{m}^{2} \mathrm{~s}^{-1} \mathrm{did}$ not produce the oscillation.

\section{$3 \cdot 3$ CONCLUSIONS}

In this chapter, we have successfully extended the E-Cell System to model RD on surface and in volume spaces with dynamic localization of molecules by implementing the Spatiocyte method. A unique feature of our method is that it can account for the important implications of volume exclusion by molecules while performing $\mathrm{RD}$ in both volume and surface spaces (see Chapter 2 and Arjunan and Tomita, 2009b). The correctness of our method and implementation is demonstrated by the accurate reproduction of the MinD and MinE oscillation behaviors in Escherichia coli, as observed in both experimental and previous computational studies. We modeled the MinDE system because the proteins display unique properties such as spatiotemporal localization patterns on the membrane and 
intercompartmental reactions. We have shown the impact of changing the various reaction and diffusion parameters to the dynamic localization patterns of the proteins. The results presented in this chapter have previously been published in (Arjunan and Tomita, 2009a). A guide to model RD systems with the implemented Spatiocyte method in the E-Cell System is also available (Arjunan, 2009).

Recent experimental studies have shown that MinE forms an annular structure around the membrane, called E-ring, which oscillates together with the polar zone (Raskin and de Boer, 1997). The work in this chapter can be further extended to model such localization patterns and elucidate the mechanism of E-ring formation, which remains ambiguous. Therefore, in Chapter 4, we propose a more detail model of the MinDE system that incorporates the formation of E-ring. We also use Spatiocyte to verify the model and uncover the mechanism of E-ring generation. 
In this chapter, using Spatiocyte we model the Escherichia coli MinDE division site control system and investigate the mechanism of MinE ring formation, which remains unclear (Lutkenhaus, 2007). The rod-shaped bacterium begins to divide when the tubulin-homolog FtsZ, polymerizes into a constricting annular structure (Z-ring) on the inner membrane (Adams and Errington, 2009; Lutkenhaus, 2007). To ensure equal distribution of cell contents to the two progeny cells, the constriction site is restricted to the middle of the cell long axis by two mechanisms: (i) nucleoid occlusion, which allows the Z-ring to assemble only at the midcell and the cell poles, locations that are devoid of the nucleoid mass (Woldringh et al., 1990, 1991; Bernhardt and de Boer, 2005), and (ii) carefully orchestrated RD of the $\min B$ operon encoded proteins, MinC, MinD, and MinE, in the cytoplasm and inner membrane, which suppresses polar Z-rings to avoid the minicelling phenotype (Lutkenhaus, 2007; Vats et al., 2009). The phenotype is characterized by the formation of chromosomeless minicells by polar septation and elongated cells with multiple chromosomes.

In the absence of MinE, the ATPase MinD in the ATP form, MinD ATP binds peripherally around the entire membrane (Raskin and de Boer, 1999). MinE, however, induces the MinD ATPase activity on the membrane, causing hydrolyzed $\operatorname{Min}_{\mathrm{ADP}}$ to be released into the cytoplasm and membrane-associated $\mathrm{MinD}_{\mathrm{ATP}}$ to localize into a polar zone that oscillates between the poles with a period of approximately $\mathrm{I}$ minute $(\mathrm{Hu}$ and Lutkenhaus, 2001). The FtsZ inhibitor, MinC piggybacks on the oscillating MinD $_{\text {ATP }}$ (Hu et al., 2003; Zhou and Lutkenhaus, 2004), ensuring that its time-averaged concentration is lowest at the midcell for the Z-ring assembly while sufficiently high at the poles to abrogate nascent FtsZ polymers (Hu et al., 1999; Hu and Lutkenhaus, 1999). 
While the exact mechanism is not known, MinE also localizes annularly to form the E-ring at the medial edge of the polar zone (Raskin and de Boer, 1997). When the E-ring is disrupted, the polar zone extends over the midcell and oscillates markedly slower, resulting in the minicelling phenotype (Raskin and de Boer, 1997; Zhang et al., 1998; Shih et al., 2002). In the absence of MinD, wild type MinE is always cytosolic. However, based on the observations from recent studies we predict that the MinE constituting the E-ring could be transiently attached to the membrane without being directly tethered to membrane-bound MinD. Firstly, doublelabel fluorescence microscopy experiments by Shih et al. (2002) clearly indicate that there is a higher density of MinE in the wild type E-ring than that of MinD at the rim of the polar zone, ruling out the possibility that all membrane-recruited MinE subunits are MinD-bound. In addition, the width of the observed E-ring, which appears to be several times the length of a MinE dimer, is also unlikely to be the direct result of MinE dimers binding to MinD subunits at the rim. Truncated MinE N-terminal domain can autonomously bind to the membrane in the absence of MinD, and in the full length MinE, the binding domain is thought to be exposed upon membrane recruitment by MinD (Ma et al., 2003).

Even though many computational models of the MinDE system have been proposed (Howard et al., 2001; Meinhardt and de Boer, 2001; Kruse, 2002; Howard and Rutenberg, 2003; Huang et al., 2003; Drew et al., 2005; Kerr et al., 2006; Fange and Elf, 2006; Pavin et al., 2006; Tostevin and Howard, 2006; Cytrynbaum and Marshall, 2007; Loose et al., 2008; Derr et al., 2009; Borowski and Cytrynbaum, 2009), the possibility that MinE is transiently attached to the membrane to form the dense E-ring has not been investigated at single molecule resolution in three-dimensional $(3 \mathrm{D})$ space. Here by accounting for the transient membrane attachment of MinE, we modeled the MinDE system with Spatiocyte and successfully reproduced the observed in vivo dynamic localization patterns. Our simulation results 
suggest that the transient membrane attachment of MinE leads to the formation of E-ring.

4.1 MODELING THE E-RING

To formulate a model of the MinDE system that can reproduce the E-ring, we looked at the properties of MinE. The domains at the two terminals of MinE appear to have separate roles in the polar zone oscillations although they have been found to interact with each other (Ramos et al., 2006). Firstly, in the absence of the MinE C-terminal domain, E-ring fails to appear (Raskin and de Boer, 1997). The C-terminal also contains the homodimerization domain of MinE (King et al., 1999). Hence, effective homodimerizations of MinE may play a significant role in E-ring, especially since it is made up of dense MinE. Secondly, when E-rings fail to appear, MinD polar zones extend beyond the midcell and take much longer to cycle between the poles (Rowland et al., 2000), resulting in the minicelling phenotype. This implies that the MinD ATPase activity is substantially reduced (Huang et al., 2003) in the absence of E-ring as a consequence of the disrupted MinE C-terminal domain. Strikingly, the MinD interaction domain resides near the MinE N-terminal (Ma et al., 2003), which was present. Therefore, because the polar zone still appeared, the ATPase activation function in the MinE N-terminal domain is likely at a very low basal level when the C-terminal domain is disrupted. Thirdly, the truncated N-terminal domain, which forms a nascent helix (King et al., 1999), can autonomously bind to the membrane in the absence of MinD (Ma et al., 2003), and in the full length MinE, the binding domain is thought to be exposed upon membrane recruitment by MinD (Ma et al., 2003). This further supports our theory that MinE constituting the E-ring remained transiently attached to the membrane independently after recruited by MinD. 
We make two assumptions to account for the above MinE properties in our model. First, since MinE cannot independently associate to the membrane, the putative membrane binding domain near the MinE Nterminal is only exposed upon binding with MinD on the membrane as suggested by Ma et al. (2003). Second, we predict that MinE can only begin to transiently attach to the membrane independently of MinD when it is in the dimer form with the membrane binding domains at both $\mathrm{N}$-terminals exposed. This is analogous to MinD, which must be in the dimer form for stable binding to the membrane (Zhou et al., 2005).

The series of reactions in the proposed model are as follows: (i) cytosolic $\mathrm{MinD}_{\text {ATP }}$ independently binds to the membrane, but with a very low affinity,

$$
\operatorname{MinD}_{\text {ATP }} \stackrel{k_{1}}{\longrightarrow} \operatorname{MinD}^{m}
$$

where the superscript $m$ denotes that the molecule is membrane bound;

(ii) $\mathrm{MinD}^{\mathrm{m}}$ cooperatively recruits cytosolic $\mathrm{MinD}_{\mathrm{ATP}}$,

$$
\operatorname{MinD}^{\mathrm{m}}+\operatorname{MinD}_{\mathrm{ATP}} \stackrel{\mathrm{k}_{2}}{\longrightarrow} 2 \mathrm{MinD}^{\mathrm{m}}
$$

(iii) MinD $^{m}$ also recruits cytosolic MinE dimer (represented as MinEE) to the membrane,

$$
\operatorname{MinD}^{m}+\operatorname{MinEE} \stackrel{k_{3}}{\longrightarrow} \operatorname{MinDEE}^{m}
$$

(iv) in the complex $\operatorname{MinDEE}^{\mathrm{m}}$, MinE stimulates MinD ATPase function, triggering the release of MinD to the cytoplasm whereas MinEE remains transiently bound to the membrane via its exposed $\mathrm{N}$-terminal membrane binding domains (Ma et al., 2003)

$$
\operatorname{MinDEE}^{\mathrm{m}} \stackrel{k_{4}}{\longrightarrow} \operatorname{MinD}_{\mathrm{ADP}}+\operatorname{MinEE}^{\mathrm{m}} ;
$$


(v) the released $\mathrm{MinD}_{\mathrm{ADP}}$ is phosphorylated in the cytoplasm,

$$
\operatorname{MinD}_{\mathrm{ADP}} \stackrel{k_{5}}{\longrightarrow} \mathrm{MinD}_{\mathrm{ATP}}
$$

(vi) the unbound MinE subunit in the $\operatorname{MinDEE}^{\mathrm{m}}$ complex can also associate with other MinD on the membrane,

$$
\operatorname{MinD}^{m}+\operatorname{MinDEE}^{\mathrm{m}} \stackrel{\mathrm{k}_{6}}{\longrightarrow} \operatorname{MinDEED}^{\mathrm{m}}
$$

(vii) the MinDEED ${ }^{m}$ complex releases $\mathrm{MinD}_{\mathrm{ADP}}$ to the cytoplasm upon activation,

$$
\operatorname{MinDEED}^{m} \stackrel{k_{7}}{\longrightarrow} \operatorname{MinD}_{\mathrm{ADP}}+\operatorname{MinDEE}^{\mathrm{m}}
$$

and (viii) because of the transient nature of $\mathrm{MinEE}^{\mathrm{m}}$ membrane association, it also dissociates to the cytoplasm

$$
\operatorname{MinEE}^{\mathrm{m}} \stackrel{\mathrm{k}_{8}}{\longrightarrow} \text { MinEE. }
$$

Note that the first five reactions, (4.1)-(4.5), are similar to the well known MinDE model by Huang et al. (2003). However, in reaction (4.4) of our scheme, the MinE dimer is transiently attached to the membrane instead of immediately becoming cytosolic after activating and releasing MinD. Table 4.1 summarizes the simulation parameters and their sources. We used the adapted NR method to execute reactions (4.4), (4.5), (4.7) and (4.8) since they are diffusion-independent. The remaining reactions were performed using the proposed method for diffusion-influenced reactions.

4.2 RESULTS

4.2.1 A wide range of model parameters reproduce MinDE oscillation dynamics

The stochastic simulation results of our wild type MinDE model are presented in Figure 4.1. The dynamic localization patterns of the oscillation 
Table 4.1: Parameters of the MinDE model to reproduce E-ring.

\begin{tabular}{|c|c|c|}
\hline Parameter & Value & Source \\
\hline$k_{1}$ & $0.022 \mu \mathrm{ms}^{-1}$ & Fitted \\
\hline$k_{2}$ & $0.03 \mu \mathrm{m}^{3} \mathrm{~s}^{-1}$ & Fitted \\
\hline$k_{3}$ & $0.5 \mu \mathrm{m}^{3} \mathrm{~s}^{-1}$ & Fitted \\
\hline$k_{4}$ & $1 \mathrm{~s}^{-1}$ & Fitted \\
\hline$k_{5}$ & $5 s^{-1}$ & (Meacci et al., 2006) \\
\hline$k_{6}$ & $5000 \mu \mathrm{m}^{3} \mathrm{~s}^{-1}$ & Fitted \\
\hline$k_{7}$ & $1 s^{-1}$ & Fitted \\
\hline$k_{8}$ & $0.83 \mathrm{~s}^{-1}$ & Fitted \\
\hline $\mathrm{D}_{\mathrm{MinD}_{\mathrm{ATP}}}, \mathrm{D}_{\mathrm{MinD}_{\mathrm{ADP}}}$ & $16 \mu \mathrm{m}^{2} \mathrm{~s}^{-1}$ & (Meacci et al., 2006) \\
\hline $\mathrm{D}_{\mathrm{MinEE}}$ & $10 \mu \mathrm{m}^{2} \mathrm{~s}^{-1}$ & (Meacci et al., 2006) \\
\hline $\mathrm{D}^{\mathrm{m}}$ & $0.02 \mu \mathrm{m}^{2} \mathrm{~s}^{-1}$ & Approximated \\
\hline Cell volume & $3.27 \mu \mathrm{m}^{3}$ & Approximated \\
\hline Cell radius & $0.5 \mu \mathrm{m}$ & Approximated \\
\hline Cell length & $4.5 \mu \mathrm{m}$ & Approximated \\
\hline$r_{v}$ & $10 \mathrm{~nm}$ & Approximated \\
\hline $\operatorname{MinD}^{\#}$ & 2000 molecules & (Shih et al., 2002) \\
\hline $\operatorname{MinE}^{\#}$ & 1400 molecules & (Shih et al., 2002) \\
\hline
\end{tabular}

The parameter value is estimated experimentally if a reference is given in the Source column. $\mathrm{D}^{\mathrm{m}}$ represents the lateral diffusion coefficient of $\operatorname{MinD}^{\mathrm{m}}, \operatorname{MinEE}^{\mathrm{m}}, \mathrm{MinDEE}^{\mathrm{m}}$ and $\mathrm{MinDEED}^{\mathrm{m}}$. The diffusion coefficient is reduced to account for membrane-bound polymerizationdepolymerization of MinD subunits (Shih et al., 2003) and the potential MinE self-oligomerization (Pichoff et al., 1995; Zhang et al., 1998), since they are not explicitly represented in the model. The approximated Escherichia coli cellular dimensions are within the typical range.

are in agreement with those observed in experiments (Raskin and de Boer, 1999; Shih et al., 2002). Moreover, dense $\mathrm{MinE}^{\mathrm{m}}$ can be seen clearly localizing annularly to form the E-ring at the edge of the polar zones (see also Figure 4.1C). The average oscillation period for 20 cycles is $48 \pm 6 \mathrm{~s}$, which is consistent with the experimentally measured average value by Shih et al. (2002). The oscillation period can also be increased to be within the range of $50 \mathrm{~s}$ to $120 \mathrm{~s}$ as experimentally determined by Meacci and Kruse (2005) by reducing the MinE transient membrane dissociation rate, $k_{8}$. For 
the model, 1 minute of simulated time takes approximately 4 minutes of runtime on an Intel Core 2 Extreme $3.2 \mathrm{GHz}$ system with $8 \mathrm{~GB}$ of RAM.

The parameters that we used for the model were obtained from experimental data if available, otherwise they were either fitted or approximated (see Table 4.1). Significantly, compared to the MinD nucleotide exchange rate used in previous models (Huang et al., 2003; Kerr et al., 2006; Fange and Elf, 2006), ours was increased by several folds to $5 \mathrm{~s}^{-1}$ to meet the lower bound determined by Meacci et al. (2006). Furthermore, increasing or reducing the fitted reaction rates simultaneously by twofold did not substantially affect the oscillation patterns but changed the average oscillation periods to $24 \pm 4 \mathrm{~s}$ and $116 \pm 23 \mathrm{~s}$ respectively. It is also possible to generate the oscillations and E-rings when the changes are larger than twofold but each fitted reaction rate needed to be readjusted. The initial positions of the molecules in the cytoplasm or on the membrane also did not have an effect in establishing and sustaining the oscillations. In addition, reducing the cell length from $4.5 \mu \mathrm{m}$ to $3.5 \mu \mathrm{m}$ or increasing the length to $5.5 \mu \mathrm{m}$ still maintained the dynamic localization patterns of MinD and MinE with the average oscillation periods of $47 \pm 6 \mathrm{~s}$ and $55 \pm 8$ $\mathrm{s}$ respectively. Taken together, a wide range of parameter values can be used to reproduce the E-ring and MinDE oscillation dynamics with our model.

4.2.2 Transient membrane attachment of MinE leads to the formation of E-ring

Figure 4.1C shows that the wild type localization patterns of MinE and MinD from our simulations are consistent with the in vivo results obtained by Shih et al. (2002). The simulated E-ring, nonetheless, is more dispersed than its in vivo counterpart possibly because we did not consider MinE oligomerization (Pichoff et al., 1995; Zhang et al., 1998) and/or MinD polymerization (Shih et al., 2003), both of which can substantially reduce the diffusion coefficient and sharply increase the subunit density at the 
localization site. In addition, a significant portion of the E-ring is composed of MinE that is transiently attached to the membrane independent of MinD (shown in cyan in Figure 4.1A and 4.1C). We conclude that our wild type model can reproduce the E-ring similar to the observations in vivo.

To confirm that our model can also reproduce the mutant phenotypes of Escherichia coli, we compared simulation results with in vivo dynamic localization patterns of MinD and MinE when the C-terminal domain of MinE is disrupted. As evident in Figure 4.2, our model accurately reproduced the disappearance of E-ring and the diffusely extended MinD polar zones observed by Rowland et al. (2000) when we reduced the MinD ATPase activation rates $k_{4}$ and $k_{7}$ to $0.06 \mathrm{~s}^{-1}$. Additionally, the oscillation also has a very long period of $\approx 10$ minutes, which corresponds to what has been observed experimentally (Rowland et al., 2000). Our results agree with previous suggestions by Huang et al. (2003) and Derr et al. (2009) that the reduction of the ATP hydrolysis rate can increase the period and reduce the intensity of E-rings. In our model, however, the E-ring is no longer visible because $\operatorname{MinE}^{\mathrm{m}}$ is dissociated much faster $\left(k_{8}\right)$ than its effective recruitment rates (arising from $k_{4}$ and $k_{7}$ ). MinE was represented as homodimers in the model although it likely existed as monomers in vivo since the C-terminal domain, required for homodimerization, was removed (Rowland et al., 2000). As a result, we readjusted the model to represent MinE as monomers, which still reproduced the mutant phenotypes when the hydrolysis rate was decreased (data not shown).

In both experiment (Shih et al., 2002) and in our simulations, the growing polar zone appears to be blocked at midcell by the E-ring before shrinking and subsequently growing again at the opposite pole. The polar zone grows because of independent nucleation and cooperative recruitment of $\mathrm{MinD}_{\text {ATP }}$ to the membrane. The shrinkage is the result of $\mathrm{MinD}^{\mathrm{m}}$ stimulation by the MinE population from the cytoplasm that is recruited to the membrane. Adding another reaction to the wild type model that allows $\operatorname{MinE}^{\mathrm{m}}$ to bind and stimulate $\mathrm{MinD}^{\mathrm{m}}$ on the membrane did not apprecia- 


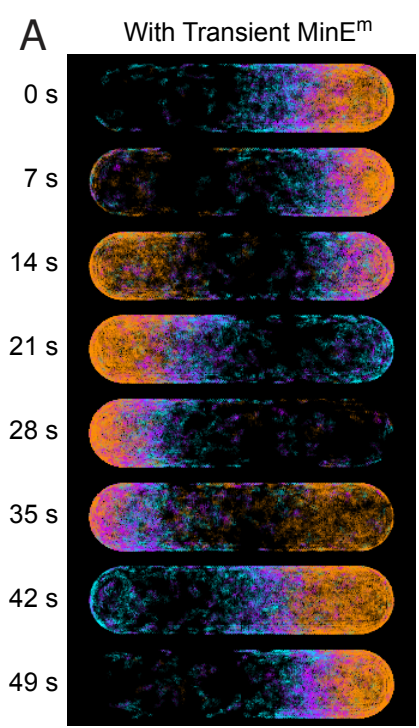

C Wild Type (Shih et al., 2002)

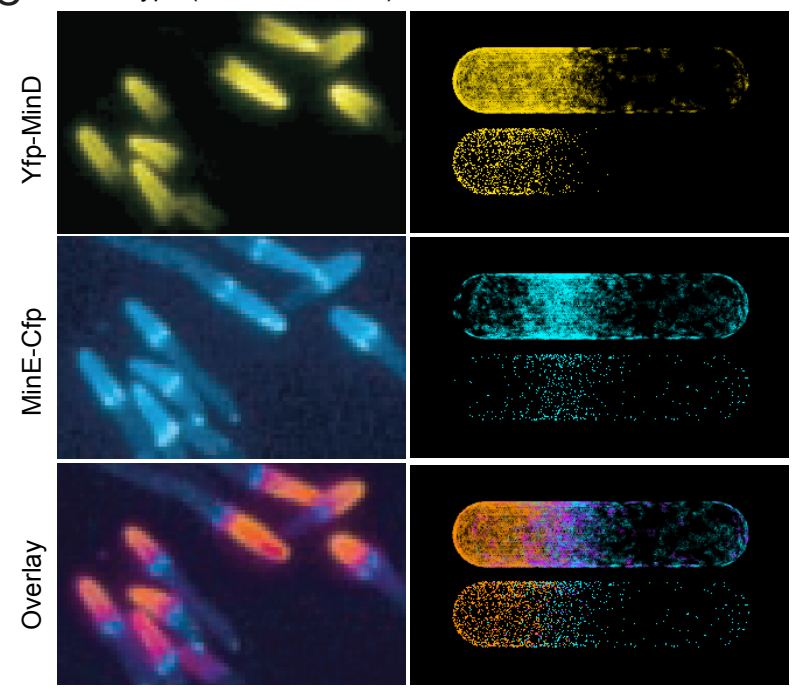

Figure 4.1: Comparison of MinDE polar zone and E-ring oscillations in wild type cells to simulation. (A) Oscillations of $\mathrm{MinD}^{\mathrm{m}}$ (orange), MinDE $^{\mathrm{m}}$ (purple) and $\mathrm{MinE}^{\mathrm{m}}$ (cyan) with transient membrane attachment of $\mathrm{MinE}^{\mathrm{m}}$ according to the proposed reactions (4.1)-(4.8) (see Table 2 for model parameters). (B) Same color scheme as in (A) but employing the adapted Huang et al. (2003) model (reactions (4.1)-(4.5)). In the Huang et al. model, reaction (4.4) is altered such that MinE is directly released to the cytoplasm together with MinD upon ATP hydrolysis instead of transiently attaching to the membrane. The reaction rates were fitted to reproduce the pole-to-pole oscillations (see text). MinE is represented as monomers in the reactions for simplicity. 
Figure 4.1: Comparison of MinDE polar zone and E-ring oscillations in wild type cells to simulation (cont.). (C) Comparison of wild type in vivo localization patterns of MinD and MinE (Left Column) to simulation (Right Column) according to our proposed model in (A). In the simulation panels, the corresponding simulated microscopy image (Upper) and single molecule visualization (Lower) are shown. In the bottom simulation panel, the molecule color scheme is as in (A). (C) (Left Column) is adapted by permission from Macmillan Publishers Ltd: The EMBO Journal (Shih et al., 2002), (c) 2002.

bly alter the dynamic localization patterns (data not shown). Therefore in vivo, the transiently attached MinEE $^{\mathrm{m}}$ could also be associating with MinD $^{m}$ directly on the membrane, bypassing the cytosolic phase.

To see if the E-ring is still generated as observed in vivo when MinE is immediately released to the cytoplasm instead of being transiently attached to the membrane upon ATP hydrolysis, we employed the adapted Huang et al. (2003) model, comprising only reactions (4.1)-(4.5). MinE was represented as monomers instead of dimers in the reactions to simplify the model and as a result, the remaining three reactions (4.6)-(4.8) were removed. In the adapted reaction (4.4), we replaced the product MinE ${ }^{\mathrm{m}}$ with cytosolic MinE to exclude transient membrane attachments of MinE. The reaction rates $k_{1}\left(0.035 \mu \mathrm{ms}^{-1}\right), \mathrm{k}_{2}\left(0.04 \mu \mathrm{m}^{3} \mathrm{~s}^{-1}\right), \mathrm{k}_{3}\left(0.5 \mu \mathrm{m}^{3} \mathrm{~s}^{-1}\right)$, $k_{4}\left(0.15 \mathrm{~s}^{-1}\right)$ and $k_{5}\left(5 \mathrm{~s}^{-1}\right)$ were fitted to reproduce the pole-to-pole oscillations. The resulting dynamic localization patterns of MinDE are shown in Figure 4.1B. The results indicate that $\mathrm{MinDE}^{\mathrm{m}}$ localized annularly at the edge of polar zones that cycled with an average period of $71 \pm 8 \mathrm{~s}$. Because the simulated MinE was always found dimerized with MinD on the membrane, the in vivo localization pattern of E-ring, which is not co-localized with MinD as shown in Figure 4.1C (Shih et al., 2002), was not reproduced. Hence, we conclude that MinE must be able to transiently attach to the membrane independently once recruited by MinD to reproduce the E-ring. 


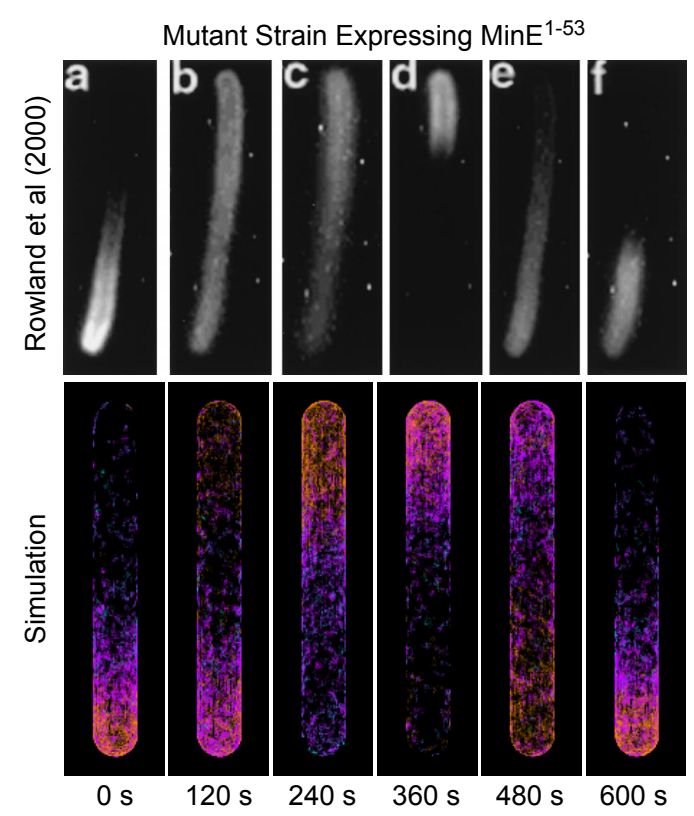

Figure 4.2: Comparison of MinDE polar zone oscillations in mutant cells to simulation. Comparison of in vivo dynamic localization patterns of Gfp-MinD (Top Row) to simulation (Bottom Row) in a mutant strain expressing $\operatorname{MinE}^{1-53}$, where the C-terminal region of MinE comprising amino acids $54-88$ was removed is shown. The mutant strain exhibits the minicelling phenotype whereby some of the cells (shown here in the Top Row) are elongated and comprise multiple chromosomes. For the simulation, the color scheme and the proposed model of MinE transient membrane association in Figure 4.I (A) were used but the MinD ATPase activation rates $k_{4}$ and $k_{7}$ were reduced to $0.06 \mathrm{~s}^{-1}$. The length of the simulated cell was increased to $8 \mu \mathrm{m}$, estimated from the mutant. The Top Row is reproduced with permission from (Rowland et al., 2000) (C) 2000, American Society for Microbiology.

\subsubsection{Width of E-ring is proportional to MinD ATPase activation rate}

The basic factors that establish and sustain in vivo MinDE oscillations according to the Huang et al. model have previously been described Huang et al. (2003). Here we examine the oscillation characteristics in terms of the adapted reaction (4.4) and the three new reactions (4.6)-(4.8) that we added to the model to generate the E-ring.

As shown in the previous section, the E-ring is formed as result of the transient membrane association of $\operatorname{MinEE}^{\mathrm{m}}$ after MinD is dissociated 
to the cytoplasm in reactions (4.4) and (4.7). Increasing the rates of ATP hydrolysis, $k_{4}$ and $k_{7}$, widened the E-ring and decreased the oscillation period. This is expected because more transiently membrane-associated $\operatorname{MinEE}^{\mathrm{m}}$ are available to form the E-ring while MinD in the polar zones is rapidly removed. Conversely, the oscillations become slower with thinner and fainter E-rings while the polar zones elongated when the rates were reduced. The fainter E-rings are consistent with the in vivo localization patterns resulting from a point mutation to the MinE C-terminal domain $\left(\mathrm{MinE}^{\mathrm{D} 45 \mathrm{~A}}\right.$ ) conducted by Shih et al. (2002). Our results thus imply that the mutation at the C-terminal domain may have impaired the MinD activation function of MinE.

Increasing the rate of reaction (4.6) narrowed the E-ring by reducing the subsequent production of transiently bound MinEE $^{m}$ dimers but decreased the period. The oscillation is rapid because more MinDEE ${ }^{m}$ associates with $\mathrm{MinD}^{\mathrm{m}}$ that are eventually activated. In reaction (4.8), increasing the rate $\operatorname{MinEE}^{\mathrm{m}}$ is dissociated to the cytoplasm also had a similar effect in that it narrowed the E-ring and made the oscillation faster. The burst in the cytosolic MinE population that are capable of rapidly rebinding with $\mathrm{MinD}^{\mathrm{m}}$ contributes to the shortening of the period. Reciprocally, E-rings widen with slower oscillations when the dissociation rate is reduced. However, if the rate is too small the E-rings become dispersed as a result of $\mathrm{MinEE}^{\mathrm{m}}$ lateral diffusion.

Overall, the population of $\operatorname{MinDEE}^{\mathrm{m}}, \mathrm{MinDEED}^{\mathrm{m}}$ and $\mathrm{MinEE}^{\mathrm{m}}$ are inversely proportional to the rates $k_{4}, k_{7}$ and $k_{8}$ respectively. The ratio of the three rates with respect to each other also generally determines the population ratio of the three different MinE species on the membrane. For example, higher $k_{4}$ and $k_{7}$ than $k_{8}$ will generate less $\operatorname{MinDEE}^{\mathrm{m}}$ and MinDEED $^{m}$ compared to MinEE $^{m}$. 


\section{$4 \cdot 3$ DISCUSSION}

In this chapter, we modeled the MinDE dynamics using Spatiocyte to elucidate the mechanism of E-ring formation, which remains ambiguous. Based on the high density of MinE in the E-ring that is not co-localized with MinD at least half of that density (assuming each MinD subunit recruited a MinE dimer at the E-ring) (Shih et al., 2002), and the ability of the truncated N-terminal domain of MinE to bind autonomously to the membrane (Ma et al., 2003), we predicted that the MinE constituting the E-ring remains transiently bound to the membrane autonomously after being recruited by MinD. To evaluate our prediction, we incorporated transient membrane attachments of $\operatorname{MinE}\left(\mathrm{MinE}^{\mathrm{m}}\right)$ in our MinDE model.

We reduced the complexity and parameters of the model by incorporating a small number of reactions that are necessary to reproduce the MinDE dynamics observed in vivo. Consequently, the reaction and diffusion parameters are effective parameters that might represent multiple processes such as oligomerization and polymerization, which are not explicitly represented. We have also made two assumptions in the model. First, as suggested by Ma et al. (2003), we assume that the putative membrane binding domain near the MinE N-terminal is only exposed upon binding with MinD on the membrane. Second, we speculate that MinE must be in a dimer form with both $\mathrm{N}$-terminal membrane binding domains exposed by MinD before it can transiently attach to the membrane independently of MinD.

With the simplification and assumptions, our model reproduced the MinDE oscillation dynamics and E-ring that are consistent with the observations in vivo. Using the Spatiocyte visualization method, we can directly compare the in vivo co-localization patterns of doubly labeled MinD and MinE (Shih et al., 2002) with simulation. Significantly, our results for the first time show that the transient membrane attachment of MinE is necessary to reproduce the E-ring as observed in vivo. As we predicted, a significant portion of the simulated E-ring consisted of transiently mem- 
brane attached $\mathrm{MinE}^{\mathrm{m}}$. The results also indicate that the width of E-ring is proportional to the MinD ATPase activation rate and inversely proportional to the membrane detachment rate of $\mathrm{MinE}^{\mathrm{m}}$. Higher binding rates of $\operatorname{MinDEE}^{\mathrm{m}}$ to $\mathrm{MinD}^{\mathrm{m}}$ also narrowed the E-ring. When the C-terminal domain of MinE was removed, the model recovered the in vivo mutant phenotypes, including the longer polar zones, the substantial delay in the oscillation period and the loss of E-ring. Our results attribute the mutant phenotypes to the reduction of MinD ATPase activation by MinE.

We suggest a possible scenario for the reduced ATPase activation rate. In the mutant, MinE is mostly found in the form of monomers because the domain responsible for dimerization near the C-terminal region has been removed. Wild type MinE in the dimer form could activate two MinD monomers concurrently and thus, it is more effective in activating multiple MinD protomers at a localized site of an oligomer or a polymer on the membrane. For example, at the leading edge of the polar zone where the E-ring forms, it is crucial for MinE to dissociate MinD faster than it is recruited to the membrane. Otherwise, it would not be possible to rapidly shrink the polar zone as seen in the wild type cells. Therefore, when the homodimerization of $\mathrm{MinE}^{1-53}$ is impaired, the effective ATPase activation rate could be significantly reduced, resulting in the mutant phenotypes.

Interestingly, MinE C-terminal point mutations of the residues Asp45 and Val49 to Ala in MinE ${ }^{\mathrm{D}_{45} \mathrm{~A} / \mathrm{V}_{49} \mathrm{~A}}$ also resulted in the minicelling phenotype (King et al., 2000; Shih et al., 2002) and the complete loss of E-rings although MinE ${ }^{\mathrm{D} 45 \mathrm{~A} / \mathrm{V}_{49} \mathrm{~A}}$ homodimerizes in vitro (Shih et al., 2002). The mutated residues form a highly conserved tetrad at the center of the MinE dimer interface King et al. (see Figure 4.3A and 200o). Even though $\mathrm{MinE}^{\mathrm{D} 45 \mathrm{~A} / \mathrm{V}_{49} \mathrm{~A}}$ might be in the dimer form in the cytoplasm and in vitro conditions that are free of membrane and MinD (Shih et al., 2002), we predict that the dimer could be destabilized and dissociated on the cell membrane by its $\mathrm{N}$-terminal interactions with dimeric or polymeric MinD 
because of the weakened mutated dimeric bonds. As shown in the secondary structure of the dimer in Figure $4.3 \mathrm{~B}$, the mutated tetrad is located at the center of solvent exposed face of the alpha helices and might be essential for the dimer stability when it associates with MinD. The proximity
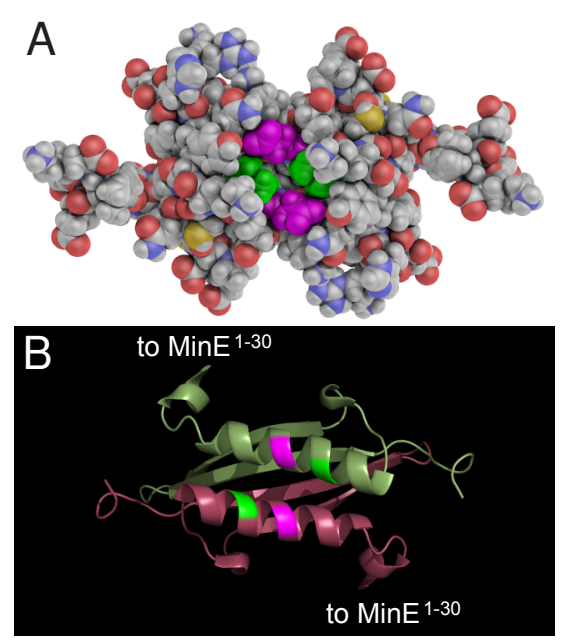

\section{C (i)} $\operatorname{Min} D=\operatorname{Min} D$ (ii)
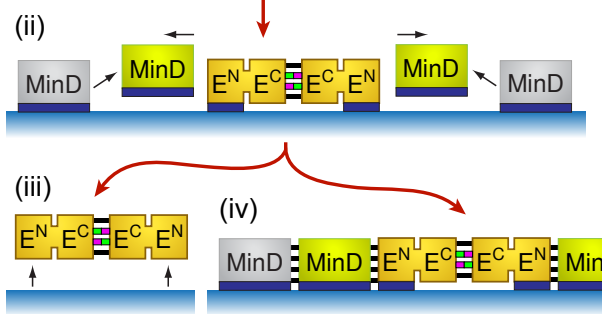

(iv)

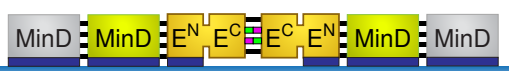

(i) MinE ${ }^{\mathrm{D} 45 \mathrm{~A} / \mathrm{V} 49 \mathrm{~A}}$ Mutant $\operatorname{Min} D \operatorname{Min} D E^{N} E^{C}-E^{C} E^{N}=\operatorname{Min} D=\operatorname{Min} D$

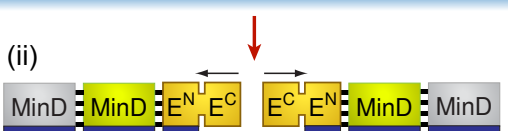

(v)

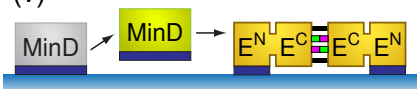

Figure 4.3: The MinE ${ }^{\mathrm{D}_{45} \mathrm{~A} / \mathrm{A}_{49} \mathrm{~A}}$ mutant dimer and molecular models of MinE interactions. (A) Space-filling representation of C-terminal MinE $^{31-88}$ homodimeric structure showing the positions of Asp45 (magenta) and Val49 (light green) residues that form a highly conserved tetrad at the center. (B) The corresponding secondary structure of MinE ${ }^{31-88}$ homodimer as shown in (A). The magenta and light green patches on the helices show the positions of Asp45 and Val49 respectively. 
Figure 4.3: The MinE $\mathrm{E}^{\mathrm{D} 4 \mathrm{~A}^{\mathrm{A}} \mathrm{A} 49 \mathrm{~A}}$ mutant dimer and molecular models of MinE interactions (cont.). (C) Molecular models of wild type MinE and MinE ${ }^{\mathrm{D} 45 \mathrm{~A} / \mathrm{A} 49 \mathrm{~A}}$ interactions in the MinDE system. The models are represented by MinD (lime green box) that could be a subunit of an oligomer/polymer (gray box), MinE N-terminal $\left(\mathrm{E}^{\mathrm{N}}\right)$ and C-terminal $\left(\mathrm{E}^{\mathrm{C}}\right)$ domains, inner membrane (light blue horizontal block), cytoplasm (white space), membrane binding domain (thick dark blue line), dimerization bond (thick black line), Asp45 (magenta box) and Val49 (light green box) dimerization interfaces, dissociation reaction (black arrow) and transition of reactions (red arrow). (Wild Type MinE)(i) Cytosolic MinE, mostly existing as dimers, is recruited to the membrane by MinD oligomers. The membrane binding domain at the $\mathrm{N}$-terminals of the dimer are exposed by MinD upon recruitment. (ii) The MinE dimer cooperatively and strongly activates MinD because both of its N-terminal domains are associated with MinD. Once MinD is released to the cytoplasm, the dimer remains transiently bound to the membrane via the membrane binding region at both of its N-terminals. The transiently bound dimer could either dissociate to the cyoplasm (iii) or associate with new MinD protomers (iv). (v) MinE dimer weakly activates the ATPase function of MinD without cooperativity when only one of its $\mathrm{N}$-terminals is associated with MinD. Once activated the dimer becomes cytosolic if the membrane binding domain is only exposed at one of its N-terminals. Reactions (i), (ii), (iii), (iv) and (v) are simplified by the reactions (4.3), (4.6), (4.7), (4.8) and (4.4), respectively, in our model. (MinE ${ }^{\mathrm{D} 45 \mathrm{~A} / \mathrm{A}} 49 \mathrm{~A}$ Mutant)(i) In the cyoplasm, $\mathrm{MinE}^{\mathrm{D} 45 \mathrm{~A} / \mathrm{A} 49 \mathrm{~A}}$ may exist as a homodimer or a monomer. The homodimer (or two monomers) is recruited to the membrane by MinD oligomers (or two monomers). The membrane-binding domain at the N-terminal of $\mathrm{MinE}^{\mathrm{D} 45 \mathrm{~A} / \mathrm{A}_{49} \mathrm{~A}}$ is also exposed with the help of MinD. (ii) The recruited MinE ${ }^{\mathrm{D} 45 \mathrm{~A} / \mathrm{A}} 49 \mathrm{~A}$ dimer is destabilized on the membrane by MinD and dissociates. (iii) The dissociated MinE ${ }^{\mathrm{D}} 45 \mathrm{~A} / \mathrm{A}_{49} \mathrm{~A}$ monomer activates its bound MinD monomer weakly since there is no cooperativity. Upon activation, both MinD and $\mathrm{MinE}^{\mathrm{D} 45 \mathrm{~A} / \mathrm{A}_{49} \mathrm{~A}}$ monomers are released into the cytoplasm. MinE ${ }^{\mathrm{D} 45 \mathrm{~A} / \mathrm{A}_{49} \mathrm{~A}}$ is not transiently bound to the membrane because it must be in the dimer form with the membrane binding domain of both monomers exposed to support transient binding. (iv and v) During the long interval to weakly activate MinD, cytosolic MinE ${ }^{\mathrm{D} 45 \mathrm{~A} / \mathrm{A}} 49 \mathrm{~A}$ monomer associates with its membrane counterpart and dissociates it to the cytoplasm. Reaction (i) and reactions (ii-v) are simplified by the reactions (4.3) and (4.4), respectively, in our model. 
of the tetrad to the N-terminal domains indicates that it could be subjected to immediate stress as MinD is associated at both ends, which could be further amplified when MinD forms higher-order complexes. Figure $4.3 \mathrm{C}$ illustrates an example of MinDE interaction model with the destabilization of MinE $\mathrm{E}^{\mathrm{D} 5 \mathrm{~A} / \mathrm{V}_{49} \mathrm{~A}}$ dimer by MinD. Additionally, a single point mutation of Asp45 to Ala in MinE ${ }^{\mathrm{D}_{45} \mathrm{~A}}$ was capable of generating narrower E-rings, which we could attribute to a less destabilized $\mathrm{MinE}^{\mathrm{D}_{45} \mathrm{~A}}$ dimer interface. Parallel to the narrowing of the E-ring width when we decreased the activation rates $k_{4}$ and $k_{7}$ in our simulation, some $\operatorname{MinE}^{\mathrm{D} 45 \mathrm{~A}}$ that are able to stay dimerized on the membrane could strongly activate MinD and form the narrower E-rings.

Since a wide range of our model parameters was able to reproduce the dynamic localization patterns of MinDE, the model could also approximate more elaborate models comprising detailed reactions such as MinDE oligomerization. In Figure 4.3 $\mathrm{C}$ we illustrate two such detailed models of wild type MinE and MinE ${ }^{\mathrm{D}_{45} \mathrm{~A} / \mathrm{V}_{49} \mathrm{~A}}$ mutant interactions in the MinDE system. Recently, Derr et al. (2009) proposed a partial non-oscillating polymerization model of the MinDE system that do not explicitly include cooperative activation of MinD to reproduce E-rings. Because in the model MinE is always found dimerized with MinD on the membrane, it cannot recover E-rings that are much denser than MinD. It will be interesting to see how polymerization of MinD affects the formation of E-ring when transient membrane attachment of MinE is incorporated. Since stronger ATPase activation would be required to fragmentize and depolymerize annealing MinD polymers than freely diffusing MinD as the case in our model, we predict denser E-ring would form at the rim of the polar zones if we extend our model to include MinD polymerization.

By observing E-ring localization patterns in fluorescence microscopy images, it is difficult to infer what causes such dynamic annular structure formation. Based on the density of MinE in the E-ring that is much higher than MinD at the rim of polar zones, we can predict that it could be caused 
by transient membrane attachment of MinE. As we showed in this work, using Spatiocyte to create a model based on the known properties of MinE and the previously reported wild type and mutant localization patterns of the MinDE system, we can substantiate our prediction. Moreover, the model also provided further mechanistic insights about the system, such as the width of E-ring could be proportional to the rate of MinD activation. 
In the previous chapter, we successfully modeled and reproduced the E-ring dynamics as observed in vivo. However, the MinDE model was simplified and did not include MinD polymerizations on the membrane. In this chapter, we extend the Spatiocyte method to model polymerization of proteins.

In addition to the spatial regulation of division site selection by the MinDE system, many other cellular processes are also regulated by protein polymers. In eukaryotic cells, cytoskeletal polymers comprising microtubules, actin and intermediate filaments participate in processes as diverse as cytokinesis, directing chromosome segregation, establishing and maintaining cell morphology, facilitating cell motility and intracellular transport, and defining cell polarity (Moseley and Goode, 2006; Herrmann et al., 2007; Verhey and Gaertig, 2007). Similar functions are also performed by bacterial cytoskeletal polymers composed of Walker A ATPases (Koonin, 1993), and homologs of tubulin, actin and intermediate filament (Michie and Löwe, 2006; Shih and Rothfield, 2006; Graumann, 2007; Pogliano, 2008). Moreover, recent research shows that several key signaling pathways in animal cells are also regulated by polymerizing proteins (Schwarz-Romond et al., 2007; Harada et al., 2008). Almost all of these polymers are dynamic, carrying out their functions through carefully orchestrated assembly, disassembly and redistribution of polymer subunits. It is becoming increasingly evident that quantitative models of polymerization are necessary to understand the collective dynamics of subunits and interacting molecules (Haviv et al., 2006; Kozlowski et al., 2007; Schek et al., 2007; Surovtsev et al., 2008; Loose et al., 2008). Polymerization models are also used to estimate subunit binding energy and activation rates (Lan et al., 2008). 
Since a polymerization model essentially involves molecular interactions, it should describe at the molecular scale the emergent properties of protein polymers that support their cellular functions (Karsenti et al., 2006). These properties include the ability of polymers to assemble cooperatively, isodesmically or both. In an isodesmic polymer, the effective binding energy for each subunit is identical and constant, whereas in a cooperative polymer, the energy is increased by lateral or allosteric interactions. Lateral cooperative assemblies produce multistranded polymers in which one or more laterally bound subunit concurrently strengthens two or more longitudinally bound subunits. In allosteric cooperative assemblies, newly bound subunits undergo conformational changes that increase their binding affinity for other subunits. The conformational changes may propagate according to the classical Monod-Wyman-Changeux (MWC) (Monod et al., 1965) or Koshland-Nemethy-Filmer (KNF) (Koshland et al., 1966) model, or the recently proposed conformational spread (CS) Bray and Duke (2004) model. A polymer elongates by recruiting subunits to one or both of its ends, or in the case of subunit treadmilling (Wegner, 1976), one end of the polymer recruits while the other dissociates them. At steady state, a constant polymer length is maintained if the rates of recruitment and dissociation are the same. When any bond between subunits is broken, the polymer becomes fragmented. Distinct or fragmented polymers may also anneal to form a contiguous polymer.

Polymer functions are also linked to its morphology and molecular interactions in the $3 \mathrm{D}$ intracellular space. Protein filaments confined to spherical or rod-shaped cell membranes are known to arrange into helical (Shih et al., 2003), ring (Bi and Lutkenhaus, 1991) and line (Larsen et al., 2007) structures. In the cytoplasm, subunits can assemble into tubules, asters and stars (Mejillano et al., 2004; Haviv et al., 2006). Intracellular spatial features that can influence polymer assembly and function include the 3 D position of subunits and polymer nucleation sites (Lutkenhaus, 2007), volume exclusion by polymer filaments (Haviv et al., 2006), molecular 
crowding (Popp et al., 2007; Wieczorek and Zielenkiewicz, 2008), intercompartmental interaction and subcellular localization of reacting molecules (Lutkenhaus, 2007), and diffusion-influenced reactions of molecules that are either small numbered or heterogeneously distributed (Shih et al., 2002; Lutkenhaus, 2007). Diffusion and small numbers of reacting molecules may also induce stochasticity (Rao et al., 2002; Bhalla, 2004; Wilkinson, 2009) in polymer assembly (VanBuren et al., 2002).

Although a variety of quantitative methods have been developed to model protein polymerization dynamics (Haviv et al., 2006; Kozlowski et al., 2007; Schek et al., 2007; Surovtsev et al., 2008; Loose et al., 2008; Cytrynbaum and Marshall, 2007; Lan et al., 2008; Miraldi et al., 2008), many of the properties above have been omitted primarily because of the computational cost and complexity. In this chapter, we extend Spatiocyte to model polymerization of proteins and show that it can incorporate the above properties while still maintaining a reasonable computational time. We focus on polymers that are constrained to surfaces such as the cell membrane, although our method can be adapted to model cytosolic polymerizations as well.

\subsection{METHODS}

To model polymerization of proteins we adopt several typical approximations in polymer models: (i) we assume that the modeled polymers are immobile and have attained their low energy morphology on the membrane; (ii) we focus on polymerizations that are free of explicit force generation and are constrained to a surface compartment; (iii) for a fragmenting polymer species, we assume that any longitudinal bond between two protomers in the polymer can break with the same rate constant; (iv) conversely for an annealing polymer species, protomers at the tip of two polymers can associate to form one longer polymer with the same rate, 
regardless of the length of the original polymers; and (v) we assume that the multistranded polymers form $2 \mathrm{D}$ sheets over a surface compartment instead of ${ }_{3} \mathrm{D}$ sheets.

In our polymerizing Spatiocyte method, a polymer is nucleated when two free monomers come into contact and associate to form an immobile dimer on the membrane. If at least one of the free monomers is a diffusing species, the monomer association is performed using the diffusion-influenced reaction approach described in Chapter 2, otherwise, the adapted NR method is applied (see Chapter 2, Section 2.3). Each monomer of the dimer, which is in contact with the other, still occupies its respective voxel and is now immobile. The dimer now constitutes the two nucleated protomers at the tips of the nascent polymer. With a given reaction rate, the protomers can each recruit another monomer longitudinally when they come into contact. As this procedure is performed repetitively, the polymer elongates by the two opposite tips to form a single stranded polymer. The polymer can be fragmented when two protomers of the polymer is dissociated. The protomers at the tip can also dissociate and become a free monomer with a given rate. All dissociation reactions are performed using the adapted NR method. Two distinct polymers can anneal when the protomers at their tips come into contact and associate. The association reaction is executed using the adapted NR method because both protomers are immobile and are in contact (see Chapter 2, Section 2.3). Thus, when a protomer is in contact with another immobile protomer that is a reactant pair, the reaction is arranged in the adapted NR method priority queue for execution according to the reaction propensity. A polymer can also become multistranded when the protomers recruit monomers laterally.

The overall geometry of a polymer is determined by the bends between each of its protomers. To constrain the polymer to the surface of a sphere or a cylinder, the typical shapes of cellular or nuclear membrane, we adopt the procedure proposed by Andrews and Arkin (2007). The procedure maps the relative turn $(\alpha)$ of a recruited protomer to the left or right of the 
recruiting protomer on a flat plane to the corresponding ${ }_{3} \mathrm{D}$ yaw $(\phi)$, pitch $(\theta)$ and roll $(\psi)$ angles for either the cylindrical or spherical membrane surface. It ensures that the two protomers are touching the curved surface and are plane-parallel to the surface below the center of the protomers. Yaw specifies the turn to the left or right of the recruiting protomer while rotating tangent to the membrane surface, pitch indicates the rotation angle up or down the recruiting protomer's frame of reference, whereas roll specifies the rotation around the polymer axis.

It is worth noting that the Andrews and Arkin procedure constrains polymers to surfaces without molecular reaction or diffusion. Moreover, the procedure was developed to constrain polymers in continuous space, unlike in the Spatiocyte method where the space is discretized into HCP lattice. As a result, we map the continuum $3 \mathrm{D}$ coordinates to the lipid voxel nearest to the target coordinates and occupy it with the recruited protomer. To prevent the accumulation of error when mapping subsequent recruited protomers from continuum to discrete space, the method keeps a copy of the initial continuum coordinates when the polymer is nucleated and uses them as the reference coordinates for subsequent recruitments. We have also added connective protomers to ensure that all protomers within a polymer is contiguous because the continuous to discrete space mapping may sometimes result in disjointed protomers.

5.2 RESULTS

\subsubsection{Spatiocyte reproduces multistranded membrane-bound polymerizations}

We evaluated Spatiocyte to nucleate a polymer on the membrane and elongate it by longitudinal recruitment of protomers at both tips of the polymer. The tip protomers correctly recruited both freely diffusing and immobile monomers from the membrane or cytoplasm. The polymer 

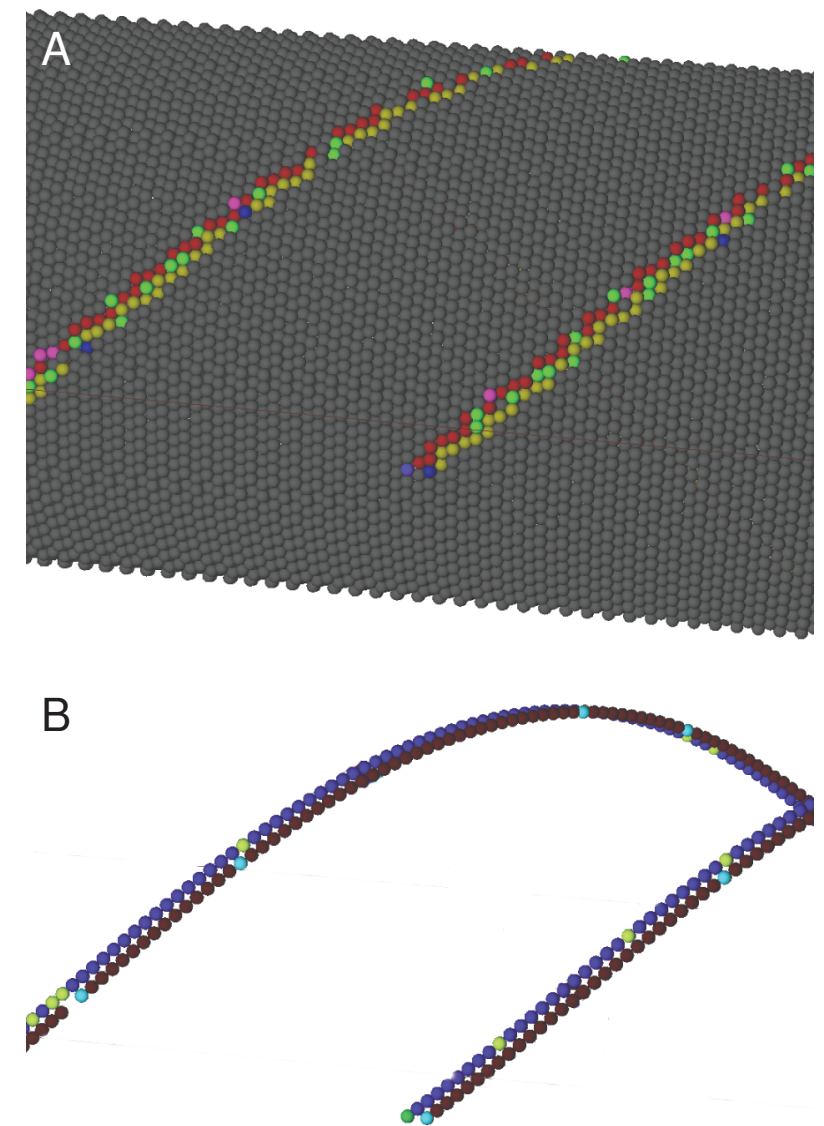

Figure 5.1: Multistranded polymerization on a cylindrical membrane. (A) Position of protomers in the HCP lattice. Lipid molecules are shown in gray. Protomers at different states, represented by different molecule species, are shown in different colors. Blue: tip protomer; pink: longitudinally bound protomer; red and olive green: laterally and longitudinally bound protomer; green: connective protomer. (B) Corresponding position of protomers shown in (A) in continuous space. Brown and blue: laterally and longitudinally bound protomer; light green: laterally bound protomer; cyan: tip protomer. 


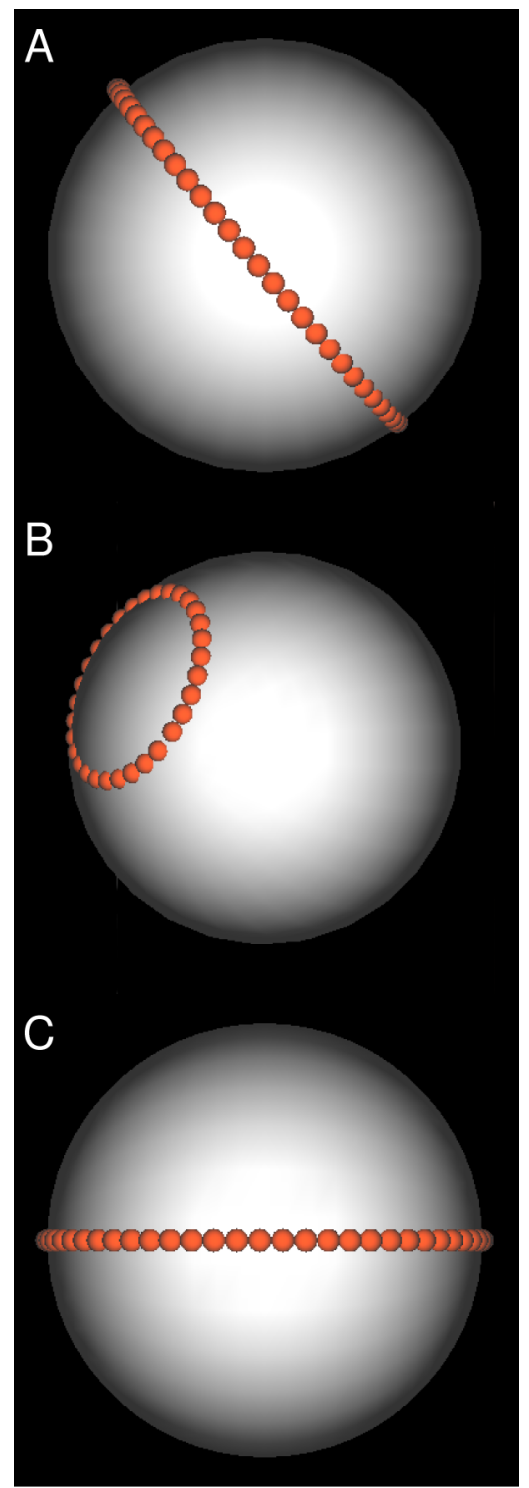

Figure 5.2: Polymer curvatures on a spherical membrane. The membrane surface is shown in white while the protomers are indicated by the small orange spheres. Position of protomers are shown in continuous space. The parameters of polymerization curvature in terms of $\{\alpha, \phi, \theta, \psi\}$ are $\left\{130,0,-1 /\left(2 r_{v}\right), 0\right\}(A),\left\{40,0,-1.5 /\left(2 r_{v}\right), 0\right\}$ (B) and $\left\{0,0,-1 /\left(2 r_{v}\right), 0\right\}$ (C). 


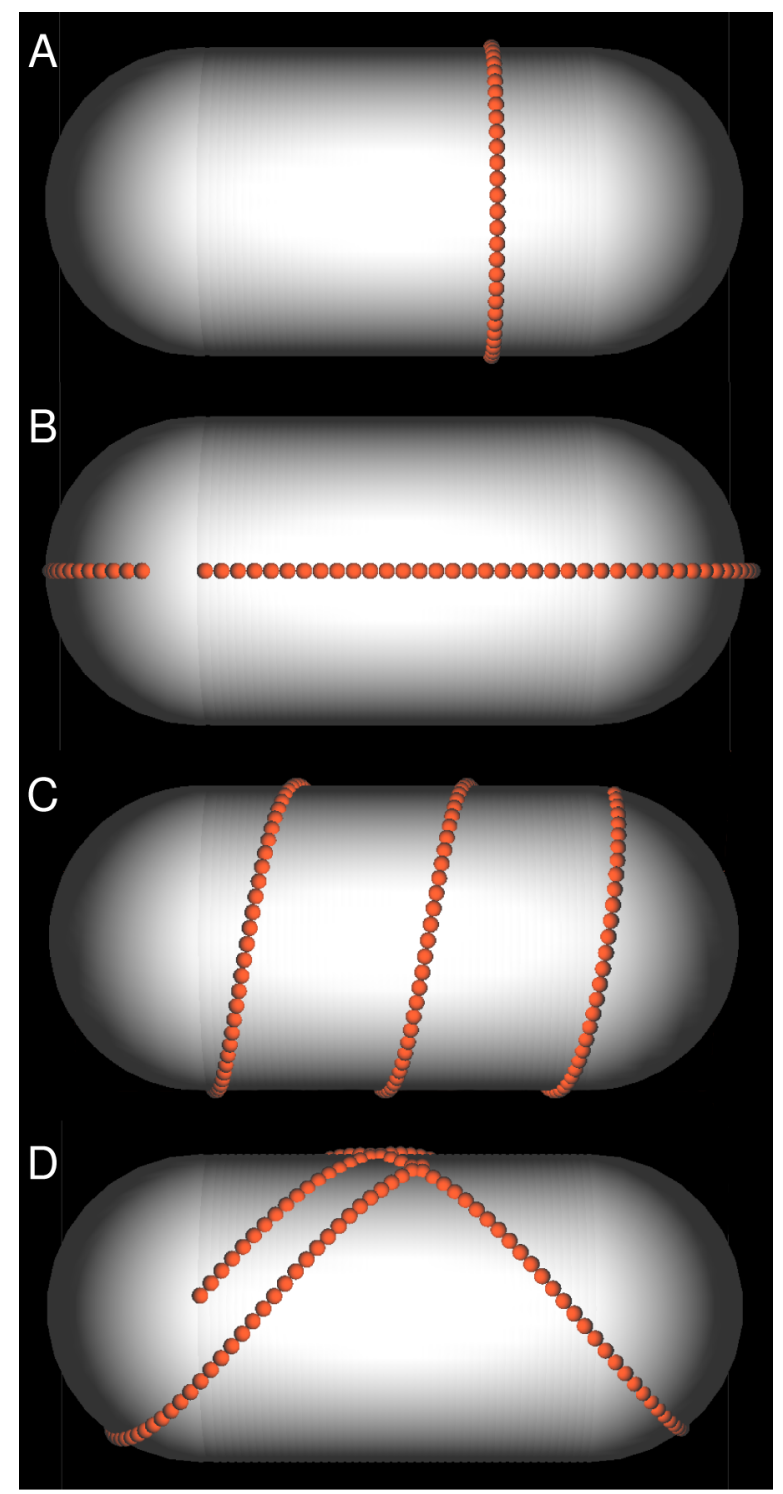

Figure 5.3: Polymer curvatures on a rod-shaped membrane. The membrane surface is shown in white while the protomers are indicated by the small orange spheres. Position of protomers are shown in continuous space. The parameters of polymerization curvature in terms of $\{\alpha, \beta, \phi, \theta, \psi\}$ are $\left\{0,90,0,-1 /\left(2 r_{v}\right), 0\right\}$ (A), $\left\{0,0,0,1 /\left(2 r_{v}\right), 0\right\}$ (B), $\left\{0,80,0.19 /\left(2 r_{v}\right),-0.96 /\left(2 r_{v}\right), 0.18 /\left(2 r_{v}\right)\right\}$ (C) and $\left\{0,46,0.64 /\left(2 r_{v}\right),-0.52 /\left(2 r_{v}\right), 0.50 /\left(2 r_{v}\right)\right\}$ (D). Here, $\beta$ is the absolute angle of the polymer on the cylinder surface, relative to the cylinder axis. 
also became multistranded when protomers started recruiting protomers laterally. Figure 5.1A shows the multistranded polymer modeled using Spatiocyte, while Figure 5.1B shows the corresponding position of the protomers in continuous space. Our method also correctly performed dissociation reactions that caused the polymer to fragmentize and shorten. Fragmented polymers also correctly annealed to form contiguous polymers when their tip protomers associated.

\subsubsection{Spatiocyte reproduces various polymer curvatures on the membrane}

Figure 5.2 and 5.3 illustrate the various polymer curvatures on the spherical and rod-shaped membranes respectively, as modeled by Spatiocyte. The figures indicate the position of polymer in continuous space. Together, these results show that Spatiocyte can accurately model membrane-bound polymerization molecules with various geometries.

\section{$5 \cdot 3$ DISCUSSION}

In this chapter, we have extended the Spatiocyte method to model membranebound polymerization of molecules. Our aim is to introduce a simple approach to represent the typical forms of membrane-bound polymerization. The method models polymerization by making several approximations as indicated in Section 5. Our results show that various polymer geometries on the membrane can be modeled by Spatiocyte. An advantage of the method is that the rate of protomer reaction or dissociation is dependent on its position, as it is the case in vivo and in vitro. For example, the fragmentation rate in the middle of the polymer is lower than the dissociation of the tip protomer, even if all protomers have the same dissociation constant. This is because dissociated protomers at the middle of the polymer have a higher chance of rebinding due to their proximity. Therefore, we 
do not need to create reactions as a function of the polymer length as it is done by other polymerization approaches (Lan et al., 2008). The method can in future be used to model the polymerization dynamics of MinD, which forms a helical structure on the Escherichia coli membrane during the polar zone oscillations. 


\section{CONCLUSIONS AND FUTURE DIRECTIONS}

In this work, we developed a new lattice-based Monte Carlo method called Spatiocyte that can perform RD in and between volume and surface compartments at single molecule resolution. Stochasticity in RD and the excluded volume effect brought by intracellular molecular crowding, both of which can significantly affect $\mathrm{RD}$ and thus, cellular processes, are also supported by Spatiocyte. To our knowledge, this is the first method that supports multicompartmental RD, stochasticity and the implications of molecular crowding simultaneously. We have verified the correctness of Spatiocyte by comparing the simulation results of diffusion, irreversible and reversible reactions with the outcomes from analytical and numerical solutions. Spatiocyte also reproduces the well known implications of molecular crowding in volume and surface compartments. To reduce the high computational cost associated with spatial modeling methods, it employs several optimization strategies. First, it can simultaneously simulate HD species, usually found in high concentrations, at compartmental scale and low copy species, often heterogeneously distributed, at molecular scale. As a result, the diffusion of each molecule belonging to the highly concentrated species, which would incur very high computational cost, can be skipped. Second, to allow bigger jumps in the simulation time, reactions involving HD species are executed in an event-driven manner. Third, the simulation space is discretized into hexagonal close-packed lattice to allow fast resolving of molecular collisions, thus supporting rapid RD simulation in crowded compartments. The optimization of computations in Spatiocyte proved to be very efficient since it only requires 4 minutes for a desktop computer to simulate 1 minute of the MinDE system that can reproduce the E-ring. 
We also devised a new visualization method that uses the temporal positional data of simulated molecules to simulate optical microscopy images of fluorescent labeled proteins. This is done by displaying the trajectory of simulated molecules averaged according to the camera exposure time used in the microphotography process. With the method we were able to directly compare our simulation results to the localization patterns of MinD and MinE in Escherichia coli and make objective evaluations.

We implemented Spatiocyte using the E-Cell System, an advanced open-source simulation platform to model and analyze both small- and large-scale biochemical reaction networks. The driver algorithm of the E-Cell System (version 3) supports concurrent executions of multiple simulation algorithms, whose time steps are independently advanced in continuous-time, discrete-time or discrete-event manner at varying timescales. Simulation algorithms can be developed using $\mathrm{C}++$ and incorporated into the system as plug in modules. By creating three basic types of algorithm modules, comprising a discrete-event simulation stepper, reaction and diffusion processes, we were able to successfully implement Spatiocyte using the E-Cell System. The correctness of the implementation was demonstrated by the accurate reproduction of the MinDE oscillation behaviors in Escherichia coli, as observed in both experimental and previous computational studies.

Using the Spatiocyte implementation we modeled the Escherichia coli MinDE division site selection system and investigated the mechanism of MinE ring formation. By observing the E-ring localization pattern in fluorescence microscopy images, it is difficult to determine what causes such dynamic annular formation. Based on the density of MinE in the Ering that is much higher than MinD at the rim of polar zones, we predicted that it could be caused by transient membrane attachment of MinE after being recruited to the membrane by MinD. As we showed in this work, using Spatiocyte to create a model based on the known properties of MinE and the previously reported wild type and mutant localization patterns of 
the MinDE system, we could substantiate our prediction. Moreover, the model also provided further mechanistic insights about the system, such as the width of E-ring could be proportional to the rate of MinD activation.

Recent experimental studies have shown that MinD forms helical polymers on the membrane (Shih et al., 2003). We have extended Spatiocyte to model such membrane-bound polymerization dynamics of molecules although it has not been used to model the MinD polymerization dynamics. In future, in addition to modeling the polymerization dynamics in the MinDE system, Spatiocyte could also be used to elucidate the formation of the division septum at the middle of Escherichia coli (Lutkenhaus, 2007; Adams and Errington, 2009) since the process also involves multicompartmental RD and polymerizations. Importantly, the FtsZ protein forms a complex polymer structure that constricts in the middle of the cell to produce the two daughter cells. To model the constriction however, Spatiocyte needs to account for the force-generation by the FtsZ polymers. Other cellular processes that are strongly dependent on the cell morphology, multicompartmental interaction and dynamic localization of molecules, such as cellular signaling (Rangamani and Iyengar, 2008) and bacterial chemotaxis (Greenfield et al., 2009; Rao and Ordal, 2009), could also be modeled in future using Spatiocyte. As the work in this thesis demonstrates, such models may provide mechanistic insights that are difficult to obtain experimentally. 
Adams, D. W. and Errington, J. Bacterial cell division: assembly, maintenance and disassembly of the $\mathrm{Z}$ ring. Nat. Rev. Microbiol., 7(9):642-653, 2009. (Cited on pages 15,58 , and 88.)

Alber, M. S., Kiskowski, M. A., Glazier, J. A. and Jiang, Y. On Cellular Automaton Approaches to Modeling Biological Cells. In Rosenthal, J. and Gilliam, D. S., editors, Mathematical Systems Theory in Biology, Communication, Computation and Finance, volume 134 of The IMA Volumes in Mathematics and its Applications, pages 1-39. Springer, New York, New York, 1st edition, 2003. (Cited on page 11.)

Allen, M. P. and Tildesley, D. J. Computer simulation of liquids. Clarendon Press, New York, NY, 1989. (Cited on page 7.)

Ander, M., Beltrao, P., Ventura, B. D., Ferkinghoff-Borg, J., Foglierini, M., Kaplan, A., Lemerle, C., Tomás-Oliveira, I. and Serrano, L. SmartCell, a framework to simulate cellular processes that combines stochastic approximation with diffusion and localisation: analysis of simple networks. Syst. Biol., 1(1):129-138, 2004. (Cited on pages 6, 12, 18, and 22.)

Andrews, S. S. and Arkin, A. P. A mechanical explanation for cytoskeletal rings and helices in bacteria. Biophys. J., 93(6):1872-1884, 2007. (Cited on pages 79 and 8o.)

Andrews, S. S. and Bray, D. Stochastic simulation of chemical reactions with spatial resolution and single molecule detail. Phys. Biol., 1(3-4): 137-151, 2004. (Cited on pages 6, 10, and 16.)

Arjunan, S. N. V. A guide to modeling reaction-diffusion of molecules with the E-Cell System. In Arjunan, S. N. V., Dhar, P. K. and Tomita, M., editors, E-Cell System: Basic Concepts and Applications, volume (In press) of Intelligence Unit. Landes Bioscience and Springer Science+Business Media, 2009. (Cited on page 57.)

Arjunan, S. N. V. and Tomita, M. Modeling reaction-diffusion of molecules on surface and in volume spaces with the E-Cell System. Int. J. Comput. Sci. Inf. Secur., 3(1):211-216, 2009. (Cited on page 57.)

Arjunan, S. N. V. and Tomita, M. A new multicompartmental reactiondiffusion modeling method links transient membrane attachment of $\mathrm{E}$. coli MinE to E-ring formation. Syst. Synth. Biol., (In press), 2009. (Cited on page 56 .)

Balgi, G., Leckb, D. and Nitsche, J. Transport effects on the kinetics of protein-surface binding. Biophys. J., 68(6):2251-2260, 1995. (Cited on page 26.) 
Baynes, B. and Trout, B. Rational design of solution additives for the prevention of protein aggregation. Biophys. J., 87(3):1631-1639, 2004. (Cited on page 8.)

Berg, H. C. Random walks in biology. Princeton University Press, Princeton, New Jersey, 1993. (Cited on page 45.)

Bernhardt, T. G. and de Boer, P. A. J. SlmA, a nucleoid-associated, FtsZ binding protein required for blocking septal ring assembly over Chromosomes in E. coli. Mol. Cell, 18(5):555-564, 2005. (Cited on pages 49 and 58.)

Bernstein, D. Simulating mesoscopic reaction-diffusion systems using the Gillespie algorithm. Phys. Rev. E, 71(4):041103, 2005. (Cited on page 22.)

Berry, $\mathrm{H}$. Monte carlo simulations of enzyme reactions in two dimensions: fractal kinetics and spatial segregation. Biophys. J., 83(4):1891-1901, 2002. (Cited on page 11.)

Bever, C. A. Selecting high-confidence predictions from ordinary differential equation models of biological networks. PhD thesis, Massachusetts Institute of Technology, 2008. (Cited on page 1.)

Bhalla, U. S. Signaling in small subcellular volumes. I. Stochastic and diffusion effects on individual pathways. Biophys. J., 87(2):733-744, 2004. (Cited on pages 3,8 , and 78 .)

Bi, E. F. and Lutkenhaus, J. FtsZ ring structure associated with division in Escherichia coli. Nature, 354(6349):161-164, 1991. (Cited on page 77.)

Borowski, P. and Cytrynbaum, E. N. Predictions from a stochastic polymer model for the MinDE protein dynamics in Escherichia coli. Phys. Rev. E, 80(4):041916, 2009. (Cited on page 59.)

Boulianne, L., Assaad, S. A., Dumontier, M. and Gross, W. GridCell: a stochastic particle-based biological system simulator. BMC Syst. Biol., 2 (1):66, 2008. (Cited on pages 6, 11, 18, and 27.)

Boyce, W. E. Elementary Differential Equations and Boundary Value Problems. Wiley, Hoboken, New Jersey, 9th edition, 2008. (Cited on page 1.)

Bray, D. and Duke, T. Conformational spread: the propagation of allosteric states in large multiprotein complexes. Annu. Rev. Biophys. Biomol. Struct., 33:53-73, 2004. (Cited on page 77.)

Brown, M. P., Grundy, W. N., Lin, D., Cristianini, N., Sugnet, C. W., Furey, T. S., Ares, M. and Haussler, D. Knowledge-based analysis of microarray gene expression data by using support vector machines. Proc. Natl. Acad. Sci. USA, 97(1):262-267, 2000. (Cited on page 1.)

Clarke, D. C. and Liu, X. Decoding the quantitative nature of TGFbeta/Smad signaling. Trends Cell Biol., 18(9):430-442, 2008. (Cited on page 15.) 
Coffey, W. T., Kalmykov, Y. P. and Waldron, J. T. The Langevin Equation: With Applications to Stochastic Problems in Physics, Chemistry and Electrical Engineering. World Scientific, Singapore, 2nd edition, 2004. (Cited on page 9.)

Collins, F. C. and Kimball, G. E. Diffusion-controlled reaction rates. J. Colloid Sci., 4(4):425-437, 1949. (Cited on pages 22 and 45.)

Conway, J. H. and Sloane, N. J. A. Sphere Packings, Lattices and Groups. Springer, New York, New York, 3rd edition, 1998. (Cited on pages 23 and 46.$)$

Cytrynbaum, E. N. and Marshall, B. D. L. A multistranded polymer model explains MinDE dynamics in E. coli cell division. Biophys. J., 93(4): 1134-1150, 2007. (Cited on pages 59 and 78.)

Derr, J., Hopper, J. T., Sain, A. and Rutenberg, A. D. Self-organization of the MinE protein ring in subcellular Min oscillations. Phys. Rev. E, 80(1): 011922, 2009. (Cited on pages 59, 65, and 74.)

Dix, J. A. and Verkman, A. S. Crowding effects on diffusion in solutions and cells. Annu. Rev. Biophys., 37:247-263, 2008. (Cited on page 15.)

Drew, D. A., Osborn, M. J. and Rothfield, L. I. A polymerizationdepolymerization model that accurately generates the self-sustained oscillatory system involved in bacterial division site placement. Proc. Natl. Acad. Sci. USA, 102(17):6114-6118, 2005. (Cited on page 59.)

Dupuy, A. D. and Engelman, D. M. Protein area occupancy at the center of the red blood cell membrane. Proc. Natl. Acad. Sci. USA, 105(8):2848-2852, 2008. (Cited on page 38.)

Economou, E. N. Green's Functions in Quantum Physics. Springer, Heidelberg, Germany, 3rd edition, 2006. (Cited on page 9.)

Einstein, A. Über die von der molekularkinetischen Theorie der Wärme geforderte Bewegung von in ruhenden Flüssigkeiten suspendierten Teilchen. Ann. Phys., 322(8):549-560, 1905. (Cited on pages 3 and 18.)

Eisen, M. B., Spellman, P. T., Brown, P. O. and Botstein, D. Cluster analysis and display of genome-wide expression patterns. Proc. Natl. Acad. Sci. USA, 95(25):14863-14868, 1998. (Cited on page 1.)

Elcock, A. Atomistic simulations of competition between substrates binding to an enzyme. Biophys. J., 82(5):2326-2332, 2002. (Cited on page 9.)

Elcock, A. Atomic-level observation of macromolecular crowding effects: escape of a protein from the GroEL cage. Proc. Natl. Acad. Sci. USA, 100 (5):2340-2344, 2003. (Cited on page 9.)

Elf, J. and Ehrenberg, M. Spontaneous separation of bi-stable biochemical systems into spatial domains of opposite phases. Syst. Biol., 1(2):230-236, 2004. (Cited on pages 6, 12, 18, and 22.) 
Ellis, R. J. Macromolecular crowding: obvious but underappreciated. Trends Biochem. Sci., 26(10):597-604, 2001. (Cited on pages 15 and 16.)

Elowitz, M. B., Surette, M. G., Wolf, P. E., Stock, J. B. and Leibler, S. Protein mobility in the cytoplasm of Escherichia coli. J. Bacteriol., 181(1):197-203, 1999. (Cited on page 4.)

Fange, D. and Elf, J. Noise-induced Min phenotypes in E. coli. PLoS Comput. Biol., 2(6):e8o, 2006. (Cited on pages 3, 15, 50, 51, 59, and 64.)

Fernández-Suárez, M. and Ting, A. Y. Fluorescent probes for superresolution imaging in living cells. Nat. Rev. Mol. Cell Biol., 9(12):929-943, 2008. (Cited on page 32.)

Friedel, M. and Shea, J. Self-assembly of peptides into a beta-barrel motif. J. Chem. Phys., 120(12):5809-5823, 2004. (Cited on page 8.)

Fulton, A. How crowded is the cytoplasm? Cell, 30(2):345-347, 1982. (Cited on page 3.)

Gibson, M. and Bruck, J. Efficient exact stochastic simulation of chemical systems with many species and many channels. J. Phys. Chem. A, 104(9): 1876-1889, 2000. (Cited on pages 12 and 21.)

Gillespie, D. T. A general method for numerically simulating the stochastic time evolution of coupled chemical reactions. J. Comput. Phys., 22(4): 403-434, 1976. (Cited on pages 12, 15, and 21.)

Gillespie, D. T. Exact stochastic simulation of coupled chemical reactions. J. Phys. Chem., 81(25):2340-2361, 1977. (Cited on pages 12 and 21.)

Graumann, P. L. Cytoskeletal elements in bacteria. Annu. Rev. Microbiol., 61:589-618, 2007. (Cited on page 76.)

Greenfield, D., McEvoy, A. L., Shroff, H., Crooks, G. E., Wingreen, N. S., Betzig, E. and Liphardt, J. Self-organization of the Escherichia coli chemotaxis network imaged with super-resolution light microscopy. PLoS Biol., 7(6):e1000137, 2009. (Cited on pages 15 and 88.)

Gómez-Uribe, C. A. and Verghese, G. C. Mass fluctuation kinetics: capturing stochastic effects in systems of chemical reactions through coupled mean-variance computations. J. Chem. Phys., 126(2):024109, 2007. (Cited on page 15.)

Hale, C. A., Meinhardt, H. and de Boer, P. A. Dynamic localization cycle of the cell division regulator MinE in Escherichia coli. EMBO J., 20(7): 1563-1572, 2001. (Cited on page 51.)

Hall, D. and Minton, A. P. Macromolecular crowding: qualitative and semiquantitative successes, quantitative challenges. Biochim. Biophys. Acta, 1649(2):127-139, 2003. (Cited on pages 4, 7, and 15.)

Hansen, C. H., Endres, R. G. and Wingreen, N. S. Chemotaxis in Escherichia coli: A Molecular Model for Robust Precise Adaptation. PLoS Comput. Biol., 4(1):e1, 2008. (Cited on page 15.) 
Harada, B. T., Knight, M. J., Imai, S., Qiao, F., Ramachander, R., Sawaya, M. R., Gingery, M., Sakane, F. and Bowie, J. U. Regulation of enzyme localization by polymerization: polymer formation by the SAM domain of diacylglycerol kinase delta1. Structure, 16(3):380-387, 2008. (Cited on page 76.$)$

Haviv, L., Brill-Karniely, Y., Mahaffy, R., Backouche, F., Ben-Shaul, A., Pollard, T. D. and Bernheim-Groswasser, A. Reconstitution of the transition from lamellipodium to filopodium in a membrane-free system. Proc. Natl. Acad. Sci. USA, 103(13):4906-4911, 2006. (Cited on pages 76, 77, and 78.)

Herrmann, H., Bär, H., Kreplak, L., Strelkov, S. V. and Aebi, U. Intermediate filaments: from cell architecture to nanomechanics. Nat. Rev. Mol. Cell Biol., 8(7):562-573, 2007. (Cited on page 76.)

Howard, M. and Rutenberg, A. D. Pattern formation inside bacteria: fluctuations due to the low copy number of proteins. Phys. Rev. Lett., 90 (12):128102, 2003. (Cited on page 59.)

Howard, M., Rutenberg, A. D. and de Vet, S. Dynamic compartmentalization of bacteria: accurate division in E. coli. Phys. Rev. Lett., 87(27): 278102, 2001. (Cited on page 59.)

$\mathrm{Hu}, \mathrm{Z}$. and Lutkenhaus, J. Topological regulation of cell division in Escherichia coli involves rapid pole to pole oscillation of the division inhibitor MinC under the control of MinD and MinE. Mol. Microbiol., 34 (1):82-90, 1999. (Cited on pages 49, 50, and 58.)

$\mathrm{Hu}, \mathrm{Z}$. and Lutkenhaus, J. Topological regulation of cell division in E. coli. spatiotemporal oscillation of MinD requires stimulation of its ATPase by MinE and phospholipid. Mol. Cell, 7(6):1337-1343, 2001. (Cited on page 58.)

Hu, Z., Mukherjee, A., Pichoff, S. and Lutkenhaus, J. The MinC component of the division site selection system in Escherichia coli interacts with FtsZ to prevent polymerization. Proc. Natl. Acad. Sci. USA, 96(26):1481914824, 1999. (Cited on pages 49 and 58.)

Hu, Z., Saez, C. and Lutkenhaus, J. Recruitment of MinC, an inhibitor of Z-ring formation, to the membrane in Escherichia coli: role of MinD and MinE. J. Bacteriol., 185(1):196-203, 2003. (Cited on page 58.)

Huang, B., Bates, M. and Zhuang, X. Super-resolution fluorescence microscopy. Annu. Rev. Biochem., 78:993-1016, 2009. (Cited on page 32.)

Huang, K. C., Meir, Y. and Wingreen, N. S. Dynamic structures in Escherichia coli: spontaneous formation of MinE rings and MinD polar zones. Proc. Natl. Acad. Sci. USA, 100(22):12724-12728, 2003. (Cited on pages $46,50,51,59,60,62,64,65,66,67$, and 68 .)

Huang, S. and Ingber, D. E. Shape-dependent control of cell growth, differentiation, and apoptosis: switching between attractors in cell regulatory networks. Exp. Cell Res., 261(1):91-103, 2000. (Cited on page 1.) 
Ideker, T. and Lauffenburger, D. Building with a scaffold: emerging strategies for high- to low-level cellular modeling. Trends Biotechnol., 21 (6):255-262, 2003. (Cited on pages 1, 2, and 15.)

Ideker, T., Galitski, T. and Hood, L. A new approach to decoding life: systems biology. Annu. Rev. Genomics Hum. Genet., 2:343-372, 2001. (Cited on page 1.)

Ji, N., Shroff, H., Zhong, H. and Betzig, E. Advances in the speed and resolution of light microscopy. Curr. Opin. Neurobiol., 18(6):605-616, 2008. (Cited on page 32.)

Karsenti, E., Nédélec, F. and Surrey, T. Modelling microtubule patterns. Nat. Cell Biol., 8(11):1204-1211, 2006. (Cited on page 77.)

Kerr, R. A., Levine, H., Sejnowski, T. J. and Rappel, W. Division accuracy in a stochastic model of Min oscillations in Escherichia coli. Proc. Natl. Acad. Sci. USA, 103(2):347-352, 2006. (Cited on pages 59 and 64.)

Kholodenko, B. N., Brown, G. C. and Hoek, J. B. Diffusion control of protein phosphorylation in signal transduction pathways. Biochem. J., 350(3):901-907, 2000. (Cited on page 3.)

King, G. F., Rowland, S. L., Pan, B., Mackay, J. P., Mullen, G. P. and Rothfield, L. I. The dimerization and topological specificity functions of MinE reside in a structurally autonomous C-terminal domain. Mol. Microbiol., 31(4):1161-1169, 1999. (Cited on page 60.)

King, G. F., Shih, Y. L., Maciejewski, M. W., Bains, N. P., Pan, B., Rowland, S. L., Mullen, G. P. and Rothfield, L. I. Structural basis for the topological specificity function of MinE. Nat. Struct. Biol., 7(11):1013-1017, 2000. (Cited on page 71.)

Kitano, H. Systems biology: a brief overview. Science, 295(5560):1662-1664, 2002. (Cited on page 1.)

Klann, M. T., Lapin, A. and Reuss, M. Stochastic simulation of signal transduction: impact of the cellular architecture on diffusion. Biophys. J., 96(12):5122-5129, 2009. (Cited on page 15.)

Koonin, E. V. A superfamily of ATPases with diverse functions containing either classical or deviant ATP-binding motif. J. Mol. Biol., 229(4):11651174, 1993. (Cited on page 76.)

Koplik, J. and Banavar, J. R. Continuum deductions from molecular hydrodynamics. Annul. Rev. Fluid Mechanics, 27(1):257-292, 1995. (Cited on page 8.)

Koshland, D. E., Némethy, G. and Filmer, D. Comparison of experimental binding data and theoretical models in proteins containing subunits. Biochemistry, 5(1):365-385, 1966. (Cited on page 77.)

Kozlowski, C., Srayko, M. and Nedelec, F. Cortical microtubule contacts position the spindle in C. elegans embryos. Cell, 129(3):499-510, 2007. (Cited on pages 76 and 78. ) 
Kruse, K. A dynamic model for determining the middle of Escherichia coli. Biophys. J., 82(2):618-627, 2002. (Cited on page 59.)

Lan, G., Dajkovic, A., Wirtz, D. and Sun, S. X. Polymerization and bundling kinetics of FtsZ filaments. Biophys. J., 95(8):4045-4056, 2008. (Cited on pages 76,78 , and 85 .)

Larsen, R. A., Cusumano, C., Fujioka, A., Lim-Fong, G., Patterson, P. and Pogliano, J. Treadmilling of a prokaryotic tubulin-like protein, TubZ, required for plasmid stability in Bacillus thuringiensis. Genes Dev., 21 (11):1340-1352, 2007. (Cited on page 77.)

Lipkow, K., Andrews, S. S. and Bray, D. Simulated diffusion of phosphorylated CheY through the cytoplasm of Escherichia coli. J. Bacteriol., 187 (1):45-53, 2005. (Cited on page 10.)

Loose, M., Fischer-Friedrich, E., Ries, J., Kruse, K. and Schwille, P. Spatial regulators for bacterial cell division self-organize into surface waves in vitro. Science, 320(5877):789-792, 2008. (Cited on pages 36, 43, 59, 76, and 78.$)$

Lutkenhaus, J. Assembly dynamics of the bacterial MinCDE system and spatial regulation of the $\mathrm{Z}$ ring. Annu. Rev. Biochem., 76:539-562, 2007. (Cited on pages 2, 15, 44, 49, 58, 77, 78, and 88.)

Löwe, J. and Amos, L. A. Evolution of cytomotive filaments: the cytoskeleton from prokaryotes to eukaryotes. Int. J. Biochem. Cell Biol., 41(2): 323-329, 2009. (Cited on page 49.)

Ma, L., King, G. and Rothfield, L. Mapping the MinE site involved in interaction with the MinD division site selection protein of Escherichia coli. J. Bacteriol., 185(16):4948-4955, 2003. (Cited on pages 59, 60, 61, and 70.$)$

Meacci, G. and Kruse, K. Min-oscillations in Escherichia coli induced by interactions of membrane-bound proteins. Phys. Biol., 2(2):89-97, 2005. (Cited on page 63.)

Meacci, G., Ries, J., Fischer-Friedrich, E., Kahya, N., Schwille, P. and Kruse, K. Mobility of Min-proteins in Escherichia coli measured by fluorescence correlation spectroscopy. Phys. Biol., 3(4):255-263, 2006. (Cited on pages 43,63 , and 64.)

Meinhardt, H. and de Boer, P. A. Pattern formation in Escherichia coli: a model for the pole-to-pole oscillations of Min proteins and the localization of the division site. Proc. Natl. Acad. Sci. USA, 98(25):14202-14207, 2001. (Cited on page 59.)

Mejillano, M. R., Kojima, S., Applewhite, D. A., Gertler, F. B., Svitkina, T. M. and Borisy, G. G. Lamellipodial versus filopodial mode of the actin nanomachinery: pivotal role of the filament barbed end. Cell, 118 (3):363-373, 2004. (Cited on page 77.)

Michie, K. A. and Löwe, J. Dynamic filaments of the bacterial cytoskeleton. Annu. Rev. Biochem., 75:467-492, 2006. (Cited on page 76.) 
Minton, A. The influence of macromolecular crowding and macromolecular confinement on biochemical reactions in physiological media. J. Biol. Chem., 276(14):10577-10580, 2001. (Cited on pages 4 and 7.)

Miraldi, E. R., Thomas, P. J. and Romberg, L. Allosteric models for cooperative polymerization of linear polymers. Biophys. J., 95(5):2470-2486, 2008. (Cited on page 78.)

Monod, J., Wyman, J. and Changeux, J. P. On the nature of allosteric transitions: a plausible model. J. Mol. Biol., 12(1):88-118, 1965. (Cited on page 77.)

Morris, D. M. and Jensen, G. J. Toward a biomechanical understanding of whole bacterial cells. Annu. Rev. Biochem., 77:583-613, 2008. (Cited on page 15.)

Moseley, J. B. and Goode, B. L. The yeast actin cytoskeleton: from cellular function to biochemical mechanism. Microbiol. Mol. Biol. Rev., 70(3): 605-645, 2006. (Cited on page 76.)

Neves, S. R. and Iyengar, R. Models of spatially restricted biochemical reaction systems. J. Biol. Chem., 284(9):5445-5449, 2009. (Cited on page 15.)

Neves, S. R., Tsokas, P., Sarkar, A., Grace, E. A., Rangamani, P., Taubenfeld, S. M., Alberini, C. M., Schaff, J. C., Blitzer, R. D., Moraru, I. I. and Iyengar, $R$. Cell shape and negative links in regulatory motifs together control spatial information flow in signaling networks. Cell, 133(4):666-680, 2008. (Cited on page 15.)

Novère, N. L. and Shimizu, T. S. STOCHSIM: modelling of stochastic biomolecular processes. Bioinformatics, 17(6):575-576, 2001. (Cited on page 18.)

Pavin, N., Paljetak, H. C. and Krstić, V. Min-protein oscillations in Escherichia coli with spontaneous formation of two-stranded filaments in a three-dimensional stochastic reaction-diffusion model. Phys. Rev. E, 73 (2):021904, 2006. (Cited on page 59.)

Pichoff, S., Vollrath, B., Touriol, C. and Bouché, J. P. Deletion analysis of gene minE which encodes the topological specificity factor of cell division in Escherichia coli. Mol. Microbiol., 18(2):321-329, 1995. (Cited on pages 63 and 64.)

Pogliano, J. The bacterial cytoskeleton. Curr. Opin. Cell Biol., 20(1):19-27, 2008. (Cited on page 76.)

Popov, A. V. and Agmon, N. Three-dimensional simulations of reversible bimolecular reactions: The simple target problem. J. Chem. Phys., 115 (19):8921-8932, 2001. (Cited on pages 29 and 37.)

Popov, A. V. and Agmon, N. Exact solution for the geminate ABCD reaction. J. Chem. Phys., 117(12):5770-5779, 2002. (Cited on page 30.) 
Popp, D., Yamamoto, A. and Maéda, Y. Crowded surfaces change annealing dynamics of actin filaments. J. Mol. Biol., 368(2):365-374, 2007. (Cited on page 78 .)

Ramaswamy, S., Tamayo, P., Rifkin, R., Mukherjee, S., Yeang, C. H., Angelo, M., Ladd, C., Reich, M., Latulippe, E., Mesirov, J. P., Poggio, T., Gerald, W., Loda, M., Lander, E. S. and Golub, T. R. Multiclass cancer diagnosis using tumor gene expression signatures. Proc. Natl. Acad. Sci. USA, 98 (26):15149-15154, 2001. (Cited on page 1.)

Ramos, D., Ducat, T., Cheng, J., Eng, N. F., Dillon, J. R. and Goto, N. K. Conformation of the cell division regulator MinE: evidence for interactions between the topological specificity and anti-MinCD domains. Biochemistry, 45(14):4593-4601, 2006. (Cited on page 60.)

Rangamani, P. and Iyengar, R. Modelling cellular signalling systems. Essays Biochem., 45:83-94, 2008. (Cited on pages 15 and 88.)

Rao, C. V. and Ordal, G. W. The molecular basis of excitation and adaptation during chemotactic sensory transduction in bacteria. Contrib. Microbiol., 16:33-64, 2009. (Cited on pages 15 and 88.)

Rao, C. V., Wolf, D. M. and Arkin, A. P. Control, exploitation and tolerance of intracellular noise. Nature, 420(6912):231-237, 2002. (Cited on pages 8 and 78.)

Raskin, D. M. and de Boer, P. A. The MinE ring: an FtsZ-independent cell structure required for selection of the correct division site in E. coli. Cell, 91(5):685-694, 1997. (Cited on pages 57, 59, and 60.)

Raskin, D. M. and de Boer, P. A. Rapid pole-to-pole oscillation of a protein required for directing division to the middle of Escherichia coli. Proc. Natl. Acad. Sci. USA, 96(9):4971-4976, 1999. (Cited on pages 50, 51, 58, and 63.)

Resch-Genger, U., Grabolle, M., Cavaliere-Jaricot, S., Nitschke, R. and Nann, T. Quantum dots versus organic dyes as fluorescent labels. Nat. Methods, 5(9):763-775, 2008. (Cited on page 32.)

Ressom, H., Reynolds, R. and Varghese, R. S. Increasing the efficiency of fuzzy logic-based gene expression data analysis. Physiol. Genomics, 13 (2):107-117, 2003. (Cited on page 1.)

Ridgway, D., Broderick, G. and Ellison, M. J. Accommodating space, time and randomness in network simulation. Curr. Opin. Biotechnol., 17(5): 493-498, 2006. (Cited on pages 2, 15, and 16.)

Ridgway, D., Broderick, G., Lopez-Campistrous, A., Ru'aini, M., Winter, P., Hamilton, M., Boulanger, P., Kovalenko, A. and Ellison, M. J. Coarsegrained molecular simulation of diffusion and reaction kinetics in a crowded virtual cytoplasm. Biophys. J., 94(10):3748-3759, 2008. (Cited on pages $4,6,10,16,29,36$, and 43 .) 
Riley, T., Sontag, E., Chen, P. and Levine, A. Transcriptional control of human p53-regulated genes. Nat. Rev. Mol. Cell Biol., 9(5):402-412, 2008. (Cited on page 1.)

Rodríguez, J. V., Kaandorp, J. A., Dobrzyński, M. and Blom, J. G. Spatial stochastic modelling of the phosphoenolpyruvate-dependent phosphotransferase (PTS) pathway in Escherichia coli. Bioinformatics, 22(15): 1895-1901, 2006. (Cited on pages 6, 12, 18, and 22.)

Rottler, J. and Maggs, A. Local molecular dynamics with coulombic interactions. Phys. Rev. Lett., 93(17):170201, 2004. (Cited on page 8.)

Rowland, S. L., Fu, X., Sayed, M. A., Zhang, Y., Cook, W. R. and Rothfield, L. I. Membrane redistribution of the Escherichia coli MinD protein induced by MinE. J. Bacteriol., 182(3):613-619, 2000. (Cited on pages 60, 65, and 68.)

Sachs, K., Gifford, D., Jaakkola, T., Sorger, P. and Lauffenburger, D. A. Bayesian network approach to cell signaling pathway modeling. Sci. STKE, 2002(148):PE38, 2002. (Cited on page 1.)

Sachs, K., Perez, O., Pe'er, D., Lauffenburger, D. A. and Nolan, G. P. Causal protein-signaling networks derived from multiparameter single-cell data. Science, 308(5721):523-529, 2005. (Cited on page 1.)

Sanford, C., Yip, M. L. K., White, C. and Parkinson, J. Cell++-simulating biochemical pathways. Bioinformatics, 22(23):2918-2925, 2006. (Cited on pages 6 and 18.)

Schaff, J., Fink, C. C., Slepchenko, B., Carson, J. H. and Loew, L. M. A general computational framework for modeling cellular structure and function. Biophys. J., 73(3):1135-1146, 1997. (Cited on page 8.)

Schaff, J. C., Slepchenko, B. M., Choi, Y., Wagner, J., Resasco, D. and Loew, L. M. Analysis of nonlinear dynamics on arbitrary geometries with the Virtual Cell. Chaos, 11(1):115-131, 2001. (Cited on pages 6 and 8.)

Schaub, S., Meister, J. and Verkhovsky, A. B. Analysis of actin filament network organization in lamellipodia by comparing experimental and simulated images. J. Cell Sci., 120(8):1491-1500, 2007. (Cited on page 42.)

Schek, H. T., Gardner, M. K., Cheng, J., Odde, D. J. and Hunt, A. J. Microtubule assembly dynamics at the nanoscale. Curr. Biol., 17(17):1445-1455, 2007. (Cited on pages 76 and 78.)

Schnell, S. and Turner, T. Reaction kinetics in intracellular environments with macromolecular crowding: simulations and rate laws. Prog. Biophys. Mol. Biol., 85(2-3):235-260, 2004. (Cited on page 11.)

Schwarz-Romond, T., Fiedler, M., Shibata, N., Butler, P. J. G., Kikuchi, A., Higuchi, Y. and Bienz, M. The DIX domain of Dishevelled confers Wnt signaling by dynamic polymerization. Nat. Struct. Mol. Biol., 14(6): 484-492, 2007. (Cited on page 76.) 
Shih, Y. and Rothfield, L. The bacterial cytoskeleton. Microbiol. Mol. Biol. Rev., 70(3):729-754, 2006. (Cited on page 76.)

Shih, Y., Fu, X., King, G. F., Le, T. and Rothfield, L. Division site placement in E.coli: mutations that prevent formation of the MinE ring lead to loss of the normal midcell arrest of growth of polar MinD membrane domains. EMBO J., 21(13):3347-3357, 2002. (Cited on pages 40, 51, 59, $63,64,65,67,69,70,71$, and 78 .)

Shih, Y., Le, T. and Rothfield, L. Division site selection in Escherichia coli involves dynamic redistribution of Min proteins within coiled structures that extend between the two cell poles. Proc. Natl. Acad. Sci. USA, 100 (13): $7865-7870,2003$. (Cited on pages $63,64,77$, and 88.)

Shimizu, T. S., Aksenov, S. V. and Bray, D. A spatially extended stochastic model of the bacterial chemotaxis signalling pathway. J. Mol. Biol., 329 (2):291-309, 2003. (Cited on page 11.)

Shreiner, D. OpenGL Programming Guide: The Official Guide to Learning OpenGL, Versions 3.0 and 3.1. Addison-Wesley, Indianapolis, Indiana, 7 th edition, 2009. (Cited on page 32.)

Slepchenko, B. M., Schaff, J. C., Macara, I. and Loew, L. M. Quantitative cell biology with the Virtual Cell. Trends Cell Biol., 13(11):570-576, 2003. (Cited on page 8.)

Smith, G. D. Numerical Solution of Partial Differential Equations: Finite Difference Methods. Oxford University Press, New York, New York, 3rd edition, 1986. (Cited on page 8.)

Stefan, M. I., Edelstein, S. J. and Novère, N. L. An allosteric model of calmodulin explains differential activation of $\mathrm{PP}_{2} \mathrm{~B}$ and CaMKII. Proc. Natl. Acad. Sci. USA, 105(31):10768-10773, 2008. (Cited on page 15.)

Stiles, J. R. and Bartol, T. M. Monte Carlo methods for simulating realistic synaptic microphysiology using MCell. In Schutter, E. D., editor, Computational Neuroscience: Realistic Modeling for Experimentalists, pages 87-127. CRC Press, Boca Raton, Florida, 2001. (Cited on pages 6, 10, and 27.)

Stundzia, A. B. and Lumsden, C. J. Stochastic simulation of coupled reaction-diffusion processes. J. Comput. Phys., 127(1):196-207, 1996. (Cited on page 12.)

Sugimoto, M. Distributed Cell Biology Simulations with the E-Cell System. In Arjunan, S. N. V., Dhar, P. K. and Tomita, M., editors, E-Cell System: Basic Concepts and Applications, volume (In press) of Intelligence Unit. Landes Bioscience and Springer Science+Business Media, 2009. (Cited on page 44.)

Sugimoto, M., Takahashi, K., Kitayama, T., Ito, D. and Tomita, M. Distributed Cell Biology Simulations with E-Cell System. In Konagaya, A. and Satou, K., editors, Grid Computing in Life Science, volume 3370/2005 of Lecture Notes in Computer Science, pages 20-31. Springer, Heidelberg, Germany, 2005. (Cited on page 44.) 
Sung, J. and Lee, S. Nonequilibrium distribution function formalism for diffusion-influenced bimolecular reactions: Beyond the superposition approximation. J. Chem. Phys., 111(3):796-803, 1999. (Cited on pages 31 and 37.)

Surovtsev, I. V., Morgan, J. J. and Lindahl, P. A. Kinetic modeling of the assembly, dynamic steady state, and contraction of the FtsZ ring in prokaryotic cytokinesis. PLoS Comput. Biol., 4(7):е1000102, 2008. (Cited on pages 76 and 78 .)

Szabo, A. Theory of diffusion-influenced fluorescence quenching. J. Phys. Chem., 93(19):6929-6939, 1989. (Cited on page 29.)

Szpiro, G. G. Kepler's Conjecture: How Some of the Greatest Minds in History Helped Solve One of the Oldest Math Problems in the World. Wiley, Hoboken, New Jersey, 1st edition, 2003. (Cited on page 18.)

Takahashi, K., Kaizu, K., Hu, B. and Tomita, M. A multi-algorithm, multitimescale method for cell simulation. Bioinformatics, 20(4):538-546, 2004. (Cited on pages 28,44 , and 47 .)

Takahashi, K., Arjunan, S. N. V. and Tomita, M. Space in systems biology of signaling pathways-towards intracellular molecular crowding in silico. FEBS Lett., 579(8):1783-1788, 2005. (Cited on pages 2, 5, 7, 15, and 16.)

Takahashi, K., Tanase-Nicola, S. and ten Wolde, P. R. Spatio-temporal correlations can drastically change the response of a MAPK pathway. Proc. Natl. Acad. Sci. USA, (In press), 2009. (Cited on page 6.)

Talbert, P. B. and Henikoff, S. Spreading of silent chromatin: inaction at a distance. Nat. Rev. Genet., 7(10):793-803, 2006. (Cited on page 44.)

Tomita, M., Hashimoto, K., Takahashi, K., Shimizu, T. S., Matsuzaki, Y., Miyoshi, F., Saito, K., Tanida, S., Yugi, K., Venter, J. C. and Hutchison, C. A. E-CELL: software environment for whole-cell simulation. Bioinformatics, 15(1):72-84, 1999. (Cited on page 44.)

Tostevin, F. and Howard, M. A stochastic model of Min oscillations in Escherichia coli and Min protein segregation during cell division. Phys. Biol., 3(1):1-12, 2006. (Cited on page 59.)

Treanor, B. and Batista, F. D. Mechanistic insight into lymphocyte activation through quantitative imaging and theoretical modelling. Curr. Opin. Immunol., 19(4):476-483, 2007. (Cited on page 15.)

van Albada, S. B. and ten Wolde, P. R. Differential affinity and catalytic activity of CheZ in E. coli chemotaxis. PLoS Comput. Biol., 5(5):e1000378, 2009. (Cited on page 15.)

van Zon, J. S. and ten Wolde, P. R. Green's-function reaction dynamics: a particle-based approach for simulating biochemical networks in time and space. J. Chem. Phys., 123(23):234910, 2005. (Cited on pages 6 and 9.) 
van Zon, J. S. and ten Wolde, P. R. Simulating biochemical networks at the particle level and in time and space: Green's function reaction dynamics. Phys. Rev. Lett., 94(12):128103, 2005. (Cited on pages 9 and 16.)

VanBuren, V., Odde, D. J. and Cassimeris, L. Estimates of lateral and longitudinal bond energies within the microtubule lattice. Proc. Natl. Acad. Sci. USA, 99(9):6035-6040, 2002. (Cited on page 78.)

Vats, P., Yu, J. and Rothfield, L. The dynamic nature of the bacterial cytoskeleton. Cell. Mol. Life Sci., 66(20):3353-3362, 2009. (Cited on page 58.)

Verhey, K. J. and Gaertig, J. The tubulin code. Cell Cycle, 6(17):2152-216o, 2007. (Cited on page 76.)

von Smoluchowski, M. Zur kinetischen Theorie der Brownschen Molekularbewegung und der Suspensionen. Ann. Phys., 326(14):756-780, 1906. (Cited on page 18.)

von Smoluchowski, M. Versuch einer mathematischen Theorie der Koagulationskinetik kolloider Lsungen. Z. Phys. Chem., 92:129-168, 1917. (Cited on pages 9 and 29.)

Wegner, A. Head to tail polymerization of actin. J. Mol. Biol., 108(1): 139-150, 1976. (Cited on page 77.)

Weimar, J. and Boon, J. Class of cellular automata for reaction-diffusion systems. Phys. Rev. E, 49(2):1749-1752, 1994. (Cited on page 11.)

Weimar, J. R. Cellular Automata Approaches to Enzymatic Reaction Networks. In Bandini, S., Chopard, B. and Tomassini, M., editors, Cellular Automata, volume 2493/2002 of Lecture Notes in Computer Science, pages 294-303. Springer, Heidelberg, Germany, 2002. (Cited on page 11.)

Weiss, M., Elsner, M., Kartberg, F. and Nilsson, T. Anomalous subdiffusion is a measure for cytoplasmic crowding in living cells. Biophys. J., 87(5): 3518-3524, 2004. (Cited on page 4.)

Wieczorek, G. A. and Zielenkiewicz, P. Influence of macromolecular crowding on protein-protein association rates-a Brownian Dynamics study. Biophys. J., 95(11):5030-5036, 2008. (Cited on page 78.)

Wilkinson, D. J. Stochastic modelling for quantitative description of heterogeneous biological systems. Nat. Rev. Genet., 10(2):122-133, 2009. (Cited on page 78 .)

Wils, S. and Schutter, E. D. STEPS: Modeling and Simulating Complex Reaction-Diffusion Systems with Python. Front. Neuroinformatics, 3:15, 2009. (Cited on page 6.)

Wishart, D. S., Yang, R., Arndt, D., Tang, P. and Cruz, J. Dynamic cellular automata: an alternative approach to cellular simulation. In Silico Biol., 5(2):139-161, 2005. (Cited on page 11.) 
Woldringh, C. L., Mulder, E., Valkenburg, J. A., Wientjes, F. B., Zaritsky, A. and Nanninga, N. Role of the nucleoid in the toporegulation of division. Res. Microbiol., 141(1):39-49, 1990. (Cited on pages 49 and 58.)

Woldringh, C. L., Mulder, E., Huls, P. G. and Vischer, N. Toporegulation of bacterial division according to the nucleoid occlusion model. Res. Microbiol., 142(2-3):309-320, 1991. (Cited on pages 49 and 58.)

Zhang, Y., Rowland, S., King, G., Braswell, E. and Rothfield, L. The relationship between hetero-oligomer formation and function of the topological specificity domain of the Escherichia coli MinE protein. Mol. Microbiol., 30(2):265-273, 1998. (Cited on pages 59, 63, and 64.)

Zhou, H. Crowding Effects of Membrane Proteins. J. Phys. Chem. B, 113 (23):7995-8005, 2009. (Cited on page 38.)

Zhou, H. and Lutkenhaus, J. The switch I and II regions of MinD are required for binding and activating MinC. J. Bacteriol., 186(5):1546-1555, 2004. (Cited on page 58.)

Zhou, H., Schulze, R., Cox, S., Saez, C., Hu, Z. and Lutkenhaus, J. Analysis of MinD mutations reveals residues required for MinE stimulation of the MinD ATPase and residues required for MinC interaction. J. Bacteriol., 187(2):629-638, 2005. (Cited on page 61.)

Zimmerman, S. and Trach, S. Estimation of macromolecule concentrations and excluded volume effects for the cytoplasm of Escherichia coli. $J$. Mol. Biol., 222(3):599-620, 1991. (Cited on pages 3, 7, and 36.) 
A

actin, 42,76

alpha helix, 72

analytical method, 29

analytical solution, 13, 86

anomalous diffusion, 36,38

aster, 77

asymptotic reaction rate, 20,21 , 30

\section{B}

Bayesian networks, I

bimolecular reaction, 19

binding energy, 77

Brownian dynamics, 2, 9

\section{C}

cell pole, 58

Cell++, 6

cellular automata, 11

Collins and Kimball, 22, 45

collision, 16, 26, 42, 45

color component, 32

compartmental scale, 18, 19

computational cost, 16, 41, 78

contact distance, 21, 30

continuous-time, 44,47

cooperative activation, 74

cooperative recruitment, 54, 65

cooperatively recruit, 61

CyberCell, 6, 10

cylindrical, 80

cytoskeletal polymer, 76

\section{D}

data structure, 47

depolymerize, 74

Diffusion Process, 48

diffusion process, 19, 29, 33

diffusion-independent reaction, 19, $21,28,29,35,36,62$

diffusion-influenced reaction, 19 , $22,27,29,36,62,78$

diffusion-limited, 15, 28, 30, 34

dimensionality, 38 dimer stability, 72

directed graph, 21

discrete-event, 12, 44, 47, 49

discrete-time, 44, 47

distributed computing, 44

driver algorithm, 47

E

E-Cell System, 28, 44, 45, 56, 87

eGFRD, 6

Einstein-Smoluchowski, 18, 46

EML, 47

E-ring, 14, 57, 59, 63

event-driven, 16, 29, 42

excluded volume, 4, 13, 15, 16, 36, 45,86

exposure time, $32,40,42$

\section{$\mathbf{F}$}

Fick's law, 12

first-order reaction, 16, 19

fluorescence microscopy, 59

fluorescent label, 32, 42, 87

fragmentation, 84

fragmentize, 74

FtsZ, 49, 58

FtsZ polymer, $5^{8}$

G

geminate pair, 31

geometry calculation, 19

GFRD, 6, 9

Gillespie algorithm, 21

GMP, 6, 12

Green's function, 9, 12, 30, 31

GridCell, 6, 27

\section{H}

helical, 85

hexagonal close-packed lattice, 16 , 42,46

hidden Markov models, 1

homodimer, 65

homodimerization, 24, 25, 71 
homogeneously distributed species, 18

\section{I}

immobile, 19, 21

indexed priority queue, 21

Intel Core 2 Extreme, 51, 64

intermediate filament, 76

intracellular viscosity, 43

intrinsic reaction rate, 21

irreversible reaction, 29, 33, 40

isodesmic polymer, 77

\section{K}

Koshland-Nemethy-Filmer, 77

\section{L}

Langevin equation, 9

Laplace space, 30

Laplace transform, 31

lateral diffusion, 43, 69

lateral displacement, 19

lattice-based method, 2, 11, 16, 41

law of mass action, 15

light diffraction, 40

lipid molecule, 18, 19

longitudinal, 78,80

\section{M}

macroscopic rate constant, 22, 27 mass action, 36

MCell, 6, 10, 27

mean squared displacement, 33,

$$
47
$$

MesoRD, 6

mesoscopic spatial scale, 11

microphotography process, 32, 42

microscopic spatial scale, 11

microscopy image, 32, 33, 40, 42

Min system, 2

minB operon, 58

MinC, 2, 44, 49, 58

MinCDE, 44

MinD, 2, 42, 44, 49, 51, 58

MinD polymerization, 64

MinD protomer, 71

MinDE, 49, 51

MinDE dynamics, 13, 70

MinDE model, 62, 76

MinDE oligomerization, 74
MinE, 2, 42, 44, 49, 51, 58

MinE C-terminal, 6o, 65

MinE dimer, 61

MinE N-terminal, 6o, 70

MinE oligomerization, 64

MinE ring, 58

minicell, 58

Model, 47

modified Bessel function, 30

molecular crowding, 3, 11, 15, 16, $36,40,41$

molecular dynamics, 2, 7

Monod-Wyman-Changeux, 77

Monte Carlo, 16, 86

MPK 1,31

multicompartmental reaction-diffusion, 16

multiparticle kernel theories, 30

multistranded, 77, 79, 84

multi-timescale algorithm, 28, 33

N

Next Reaction method, 12, 21, 62, 79

nucleoid occlusion, 44, 49, 58

numerical method, 29

numerical solution, 13, 86

O

off-lattice, 16, 36

opacity, 32

OpenGL, 32

open-source, 44

ordinary differential equations, 1

oscillation, 44, 50, 52, 62, 69, 70

\section{P}

partial differential equations, 1,8

period, 51, 53, 58, 63

pitch, 80

point mutation, 71

polar zone, 49, 52, 57, 59

polymerization, 49,76

polymerization model, 74

polymerize, 58

priority queue, 28,48

Process, 47

pseudo first-order reaction, 19

$\mathbf{R}$

RAM, 51 
random number, 18, 22

random walk, 19, 45

Reaction Process, 48

reaction-diffusion method, 18

reactive collision, 22,28

rebinding, 69

reflective boundary condition, 30

reversible reaction, 30, 34

RGBA, 32

roll, 80

S

second-order reaction, 16, 21, 22

simulated image, 40

single molecule resolution, 32

SmartCell, 6, 12

Smoldyn, 6, 10

Smoluchowski, 9, 10, 29

spatial Gillespie, 2, 12, 22

Spatiocyte, 6, 13, 16, 33, 36, 45, 56

spherical, 80

step interval, 47

Steppers, 47

STEPS, 6

stochastic, 44, 62

stochasticity, 12, 15, 41, 78, 86

Stokes-Einstein relation, 3

surface compartment, 16, 21, 25

surface reaction, 29, 30

survival probability, 29, 30, 33, 36, 40

\section{T}

tetrad, 71

three-dimensional space, 59

time-driven, 16

tip protomer, 84

trajectory, 32, 40, 42

transient membrane attachment,

$$
59,70,74
$$

transparency, 32

treadmilling, 77

tubulin, 76

U

uniform distribution, 18

unimolecular reaction, 19

V

Variable, 47
Variable Reference, 47

Virtual Cell, 6, 8

visualization method, 32, 40, 42

volume compartment, 16

volume reaction, 29

W

walk probability, 28, 33

Walker A ATPase, 76

$X$

XML, 47

Y

yaw, 8o

Z

Z-ring, 58 